NBSIR 82-2449

\title{
Technical Activities 1981 Office of Nondestructive Evaluation
}

U.S. DEPARTMENT OF COMMERCE National Bureau of Standards

National Measurement Laboratory

Office of Nondestructive Evaluation

Washington, DC 20234

December 1981

n-^oared for

j. DEPARTMENT OF COMMERCE tional Bureau of Standards shington, DC 20234 

$\ldots$

TECHNICAL ACTIVITIES 1981

OFFICE OF NONDESTRUCTIVE

EVALUATION

Harold Berger and Leonard Mordfin, Editors

U.S. DEPARTMENT OF COMMERCE

National Bureau of Standards

National Measurement Laboratory

Office of Nondestructive Evaluation

Washington, DC 20234

December 1981

Prepared for

U.S. DEPARTMENT OF COMMERCE

National Bureau of Standards

Washington, DC 20234

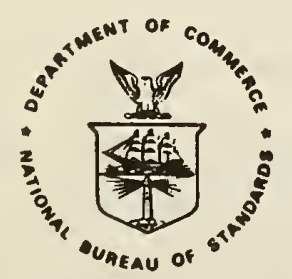

U.S. DEPARTMENT OF COMMERCE, Malcolm Baldrige, Secretary NATIONAL BUREAU OF STANDARDS, Ernest Ambler, Director 


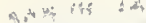

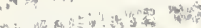

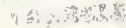

ron 
Table of Contents

Page

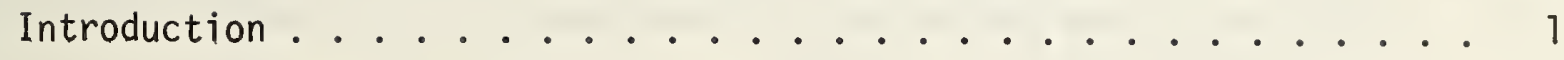

Technical Progress Reports

1. Acoustic Emission for NDE ---- J. A. Simmons, R. B. Clough, and

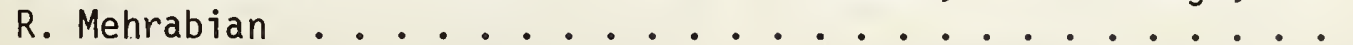

2. Electric Power Research Institute/National Bureau of Standards Joint Program on Acoustic Emission -..- D. Eitzen,

F. Breckenridge, N. Hsu, T. Proctor, R. Clough, and J. Simmons. . 7

3. NBS Conical Transducer: A Quantitative Acoustic Emission Sensor -.- T. M. Proctor ............. 23

4. Electrochemical Noise --.- U. Bertocci and J. Kruger. . . . . 28

5. Characterization Kinetics Study of Amorphous Pd-Cu-Si by UTtrasonic Measurements ---- M. Rosen and R. Mehrabian . . . . . 29

6. Nondestructive Evaluation of Composites ---- H. M. Ledbetter, J. C. Moulder, and S. K. Datta ........... 32

7. Application of NDE to Construction/Army --.- J. R. Clifton and

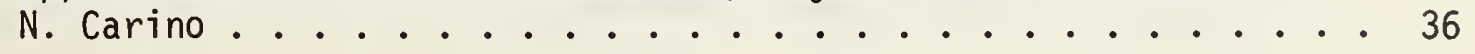

8. Monitoring of Segregation Processes in Metals by Electrical Resistivity -.. M. B. Kasen. .......... 38

9. Electrical Conductivity Standards --.- A. J. McAlister. . . . 40

10. Eddy Current Standards and Instrumentation ---- G. Free and

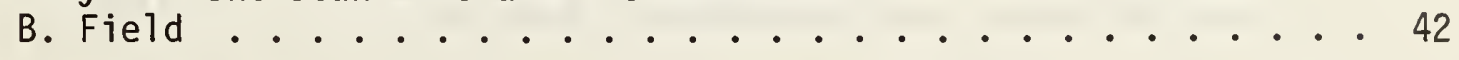

11. Theoretical Modelling in Eddy Current NDE ---- A. H. Kahn ... 47

12. Leak Rate Reference Standards ---- S. Ruthberg. . . . . . . . 50

13. Magnetic Measurements -.-- L. J. Swartzendruber . . . . . 54

14. Optical Measurement of Surface Roughness ---- E. Teague, T. Vorburger, F. Scire, S. Baker, and C. Trahan ....... 57

15. Optical Scattering for Nondestructive Evaluation ---- A. Feldman

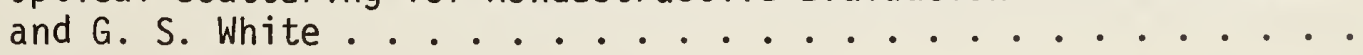


16. Signatures for Holographic NDE -.-- C. M. Vest . . . . . . .

17. Fluid Dynamics of Liquid Penetrant Flows ---- J. M. McMichael . .

18. Use of Uranium-Doped Glasses for the Calibration of Apparatus Employed in the Evaluation of Fluorescent Dye Penetrants -... R. W. Burke and D. K. Hancock .............

19. Mechanical Properties Characterization for Quality Control and NDE of Polymers -.-- D. L. Hunston and J. L. Rushford . . . . . 7 A

20. Polymer Transducers _...- S. Edelman and S. C. Roth ....... 77

21. Doppler-Broadened Positron Annihilation Lineshape for Detection of Defects in Materials -..- R. C. Reno and L. J. Swartzendruber.

22. Scattering of Acoustic Waves --- R. D. Mountain ......

23. U1trasonic Imaging/Instrumentation -..- M. Linzer and

S. J. Norton ................

24. U1trasonic Reference Block Standards and Measurements --.--

G. V. Blessing ...............

25. Laboratory Evaluation of U1trasonics for Crack Detection in Concrete ---. L. I. Knab and J. R. Clifton ........

26. Reference Samples for U1trasonic Residual Stress Measurement ---N. Hsu, T. Proctor and G. Blessing ..........

27. Nondestructive Evaluation of Nonuniformities in 2024 and 2219

Aluminum A7loy Plate--Relationship to Processing/NASA ----

L. K. Ives, L. J. Swartzendruber, W. J. Boettinger, M. Rosen,

S. D. Ridder, F. S. Biancaniella, R. C. Reno, S. Fick,

D. B. Ballard, and R. Mehrabian

28. Safety Factors and Mathematical Modeling ---- J. T. Fong ....

29. Visual Acuity Requirements for Nondestructive Testing ----

G. Yonemura ................. 94

30. Wear Condition Monitoring -..- A. W. Ruff . . . . . . . . 97

31. X-Radiography --.- R. Placious, D. Polansky, C. Dick, and J. Motz

32. Comparison of Non-Reactor Based Neutron Radiographic Facilities -.-. W. L. Parker and D. A. Garrett . . . . . . . . . . 
33. X-Ray Residual Stress Mapping in Industrial Materials by Energy Dispersive Diffractometry -..- M. Kuriyama, W. Boettinger, and

C. Bechtoldt. ..................... 105

Appendices

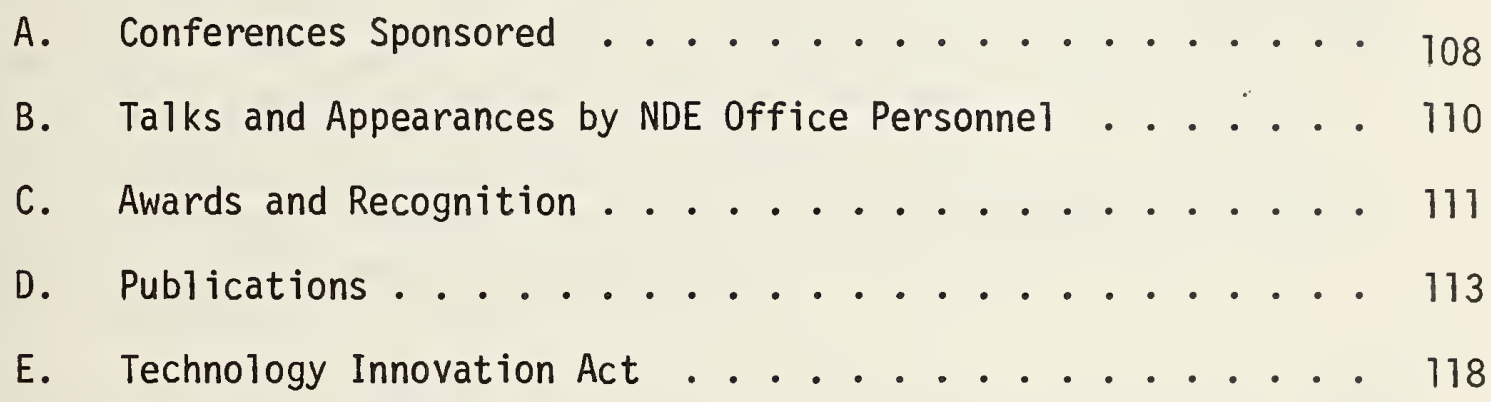





\section{INTRODUCTION}

Nondestructive evaluation (NDE) involves many measurement methods that are used to examine materials or assemblies in such a way that the tested object can be used after the test is finished. This is in contrast to destructive tests in which the tested object is destroyed in order to obtain the required information, e.g., a tensile test in which the object is pulled apart. NDE methods vary from straightforward visual inspection to sophisticated approaches in which three-dimensional images of the interior of objects are reconstructed from x-ray, neutron or ultrasonic measurements. These kinds of tests, involving optical, radiographic, magnetic, electrical, ultrasonic and other similar phenomena, are chosen and used so as not to impair the usefulness of the tested object. The commonly used methods are listed in Table I.

There are several reasons why NDE is important. Since the test object is not destroyed, $100 \%$ inspection is possible and may be desirable in many cases. Critical components involving safety considerations, such as primary components of an airplane, should all be tested. In this example, one can recognize some of the growing interest in NDE because of product liability problems. Liability considerations are encouraging many manufacturers of consumer products to use NDE more effectively.

There are other incentives leading to increased use of NDE. If we can be assured that a component wi 17 contain defects such as inclusions, cracks or voids only below a certain size, then the design can be tightened. This can result in savings of material, energy and time. The impact is both on conservation and productivity. Furthermore, there is a strong trade incentive for manufacturers to produce quality products and components. Competition at home and abroad is increasing. Many U.S. markets have fallen not because of price but because of a perception of quality. Therefore, NDE measurements in industry are, and will continue to be, on the increase because of pressures from productivity, conservation, product liability and competition.

Standards and calibration fill a very necessary role because ther is a natural desire for accurate NDE measurements - and because customers expect and often require traceable measurements. Traceability for many NDE measurements is now available in the form of calibrations and Standard Reference Materials (SRM's) from NBS. Traceable measurements include calibration services for acoustic emission and ultrasonic transducers and ultrasonic reference blocks. There are many NDE related SRM's available including those for thickness and wear measurement, radiographic density and newly announced SRM's, penetrant sensitivity plate (SRM 1850), and a silicon resistivity standard for eddy current testing (SRM 1523). Plans include SRM's for eddy current conductivity measurements, for fluorescent brightness and for radiographic sensitivity. Further information about traceable measurements is available (see, "A Basic for Traceable NDE Measurements" NBSIR 80-2109, December 1980). 
The current status of the NBS program in NDE, including work toward traceable measurements and improved procedures for NDE is described in the technical reports that follow. Those desiring additional information about the NDE activities at NBS are encouraged to contact the Office of NDE. If your inquiry is about a specific technical report, inquiries directly to the investigator/author are encouraged. 


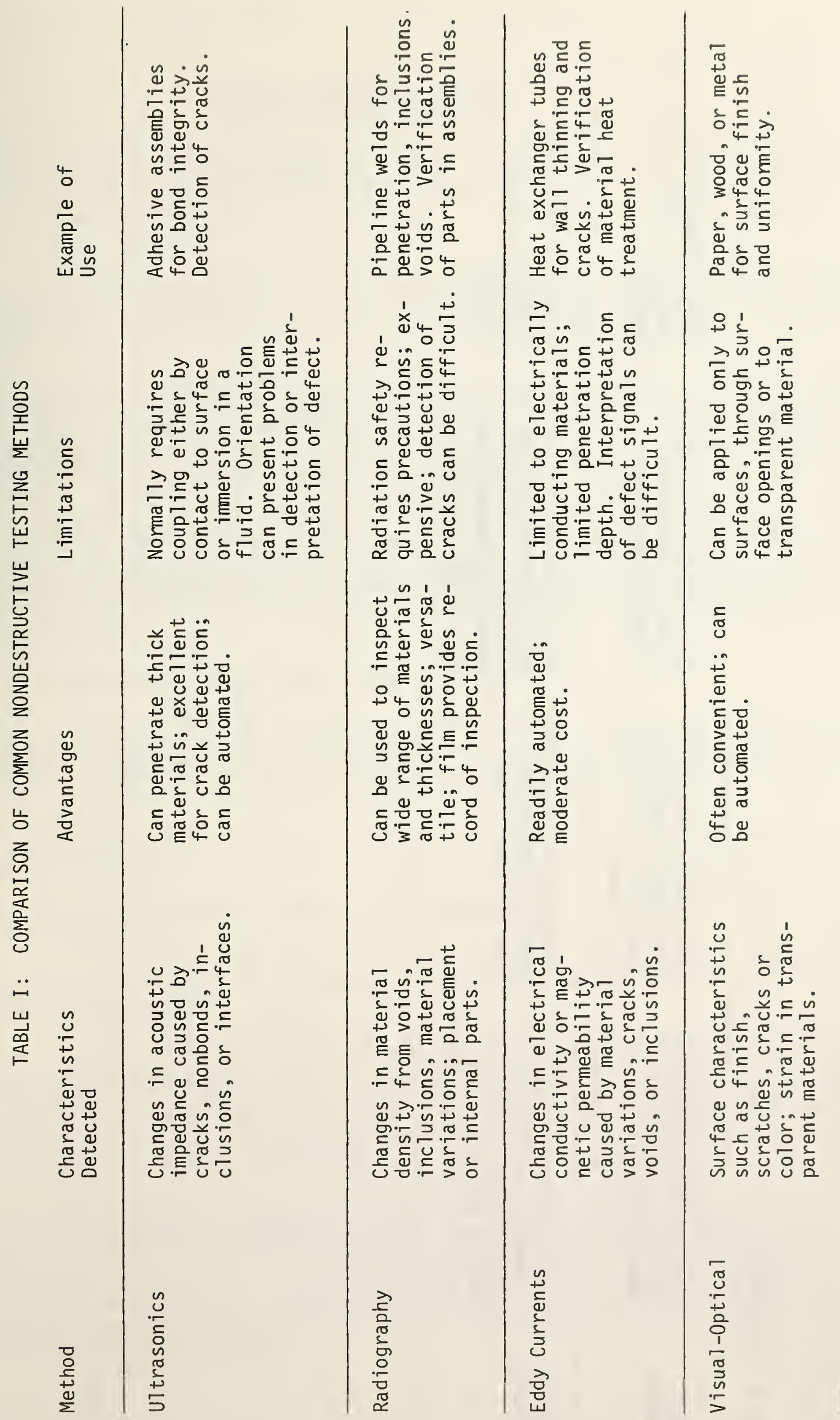




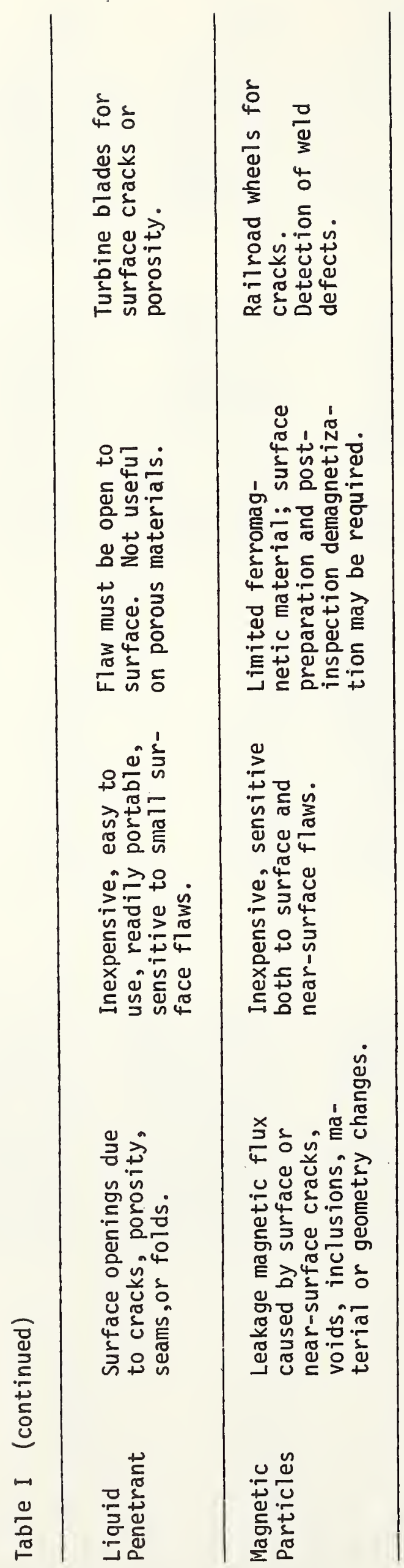




\section{TECHNICAL PROGRESS REPORTS}

1. Acoustic Emission for NDE

J. A. Simmons, R. B. Clough and R. Mehrabian

Metallurgy Division

Center for Materials Science

Acoustic emission (AE) testing shows promise for monitoring flaw growth in structures and also for materiais studies of rapid phase changes such as martensitic transitions and microcrack nucleation and growth.

At NBS a unique approach is being developed for acoustic emission which has as its objective the reconstruction of the source in terms of its stress drop tensor. This will make accessible quantitative observations on dynamic materials phenomena such as rapid solidification, martensitic phase transitions, microcrack nucleation and devitrification of glasses.

Because of the tensor nature of the emission source, at least six observation channels are necessary for source reconstruction via signal inversion. With this in mind, a multichannel $A E$ system has been constructed and new experimental methods are being developed to permit us shortly to perform a set of critical experiments which will establish the validity of this approach.

Highlights of these activities include:

(1) Application of indentation acoustic emission, the new experimental technique developed here, to A533B pressure vessel steel; through modification of heat treatment and hydrogen charging, AE activity can be varied over the widest possible limits, from virtualiy none to that of glass.

(2) Construction of the quantitative multichannel AE source characterization system.

(3) Experimental simulation of thermoelastic AE sources using a Nd-YAG laser.

(4) Exploratory AE studies of rapid solidification using directed high energy sources.

The first two results are part of a continuing EPRI research program and are described on pages 11 and 12. The third was part of a guest worker program with AERE Harwell in which Dr. Haydn Wadley took part. The last is part of a new program with DARPA that makes use of sophisticated new NDE techniques to study rapid solidification as a dynamic process as well as to detect defects that may be produced during solidification.

Experimental AE Source Simulation. A Nd-YAG laser is being used as a highly reproducible, thermoelastic, AE source for simulating defects such as microcrack dipoles in a systematic set of studies. The reproducibility of the source permits the use of a single channel for mapping the stress wave fields generated by the thermoelastic source. These experimental results will be compared with theoretical curves generated from computer codes that were developed previously. 
Rapid Solidification Studies. AE has great potential for the study of dynamic materials events such as rapid solidification, where stress waves may be generated by thermoelastic contractions, by phase change contractions, by defects such as cracks produced during solidification, or by other solidification phenomena.

Virtually no information of this type has been obtained for rapid solidification. Two approaches are being taken; the first is a wideband technique for use with a pulsed laser, where solidification can be kept rapid enough to capture the virgin stress waves before reflected waves distort the signal. This technique would be useful for solidification events less than about $100 \mu \mathrm{s}$ in length. So far this approach has been limited to detection of vaporization phenomena. The second technique is a low frequency, narrow bandwidth method which is suited for solidification phenomena of duration of the order of millisecond or more, such as are obtainable with a steadystate melt generated by an electron beam. For these conditions, exploratory measurements have been made which show transducer ringing, a sign of acoustic rather than electrical (noise) phenomena. Conditions are being established to optimize the signal-to-noise ratio and to verify the phenomena. Verification can be accomplished, in part, by comparing signals generated in the solid state by thermoelasticity with signals generated during solidification from a steady-state melt following the termination of the beam current. 
2. Electric Power Research Institute/National Bureau of Standards Joint Program on Acoustic Emission

D. Eitzen, F. Breckenridge, N. Hsu and T. Proctor

Mechanical Production Metrology Division

Center for Manufacturing Engineering

and

R. Clough and J. Simmons

Metallurgy Division

Center for Materials Science

Acoustic emission (AE) techniques have great potential for determining and monitoring structural integrity. Acoustic emission signals contain potentially useful information about the location and intensity of defects and about the criticality of the defects in a structure under load. However, current signal reception methods do not preserve all of the available information, and signal processing techniques such as threshold counting, RMS recording, energy measurement, peak detection, and spectral analysis often fail to extract the remaining information unambiguously. The difficulty in interpreting AE signals lies in the inherent complexity of the generating mechanisms, the transient wave propagation details, and the physics of the mechanical-to-electrical conversion process of the sensor.

The objective of the EPRI/NBS Joint Program on Acoustic Emission is to develop certain of the information needed to form a basis for the AE monitoring of nuclear reactor structures and to establish the feasibility of AE monitoring in a simple structure. The achievements of the first phase of the program were summarized in (1) and included:

- contributions to data interchangeability for laboratory and field measurements through the development of a transducer calibration service

- contributions to the reliability of $A E$ field data through the development of calibrated sources

- a better understanding of AE source behavior through theoretical predictions of the behavior of AE sources

- a better understanding of the limitations of conventional AE methods through a critical analysis

- a framework for understanding the AE process from source to measured voltage

- an analysis of the potential information in AE signals, an understanding of the modification to signals by wave propagation, experimentally verified theoretical predictions of remote displacement due to an AE source, and part of the basis for secondary sensor calibrations, all through the theoretical solution for wave propagation in a plate

- demonstration of the feasibility of determining the-actual AE source function in glass and in steel from remote measurements using time-domain deconvolution

- development of a methodology for determining the significance of defects from received $A E$ signals

- development of several additional very promising signal processing techniques. 
Some of these developments are being used in industrial applications and others are being used by other AE researchers. While these accomplishments are consistent with the technical plan, additional information must be developed before utility power plant operators can obtain all the information from $A E$ that is desired.

In this report we summarize our most recent work towards quantitative acoustic emission measurements within the EPRI/NBS AE Program. The acoustic emission work we describe here focuses on three areas:

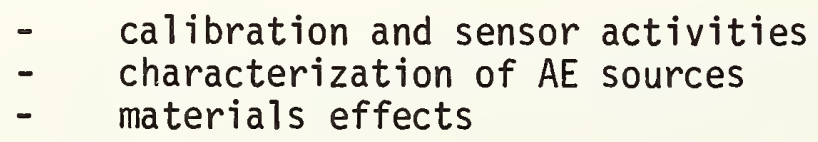

\section{CALIBRATION AND SENSOR ACTIVITIES}

In January 1980 we implemented a calibration service for acoustic emission transducers using the surface-pulse method. Since then almost 200 calibrations have been performed, 68 for other laboratories, 40 for internal quality control or improvements, 15 for other NBS units and about 70 for the development of the NBS Conical Transducer. This calibration method makes use of the theoretical solution for the normal displacement of a half-space due to a point-force step-function. The surface-pulse calibration method also relies on our ability to mechanically produce a point-force step-function (we use the breaking glass capillary method (2-4)) and it relies on an accurate measurement of actual surface displacement using a capacitive transducer (5).

\section{Further Developments of the Surface-Pulse System}

Repeatability of calibration has been a problem with certain types of transducers. Al though most transducers can be calibrated repeatably without using any special or unusual mounting practices, there are some which apparently require special measures to insure repeatability of the calibration. The measures which we have developed are, in our experience, sufficient to guarantee reproducible calibrations, although they are not always necessary. The reason why some transducers require these measures, and others do not, is not clear to us at this time. We suspect that porosity of the wear face may cause air entrapment and resulting bubbles in the couplant layer.

Special measures used for troublesome transducers include:

- the wear face of the transducer was optically ground and polished to within approximately 3 fringes of being flat

- any burrs on the steel block at the transducer mounting location were removed by hand lapping. The criterion here is that an optical flat should register no more than one half of fringe of separation when held down by gravity

- a clock oil was used as the couplant

- great care was taken in the mounting of the transducer to exclude dirt

- a special hold-down device was used, which applied a downward force ( $1 \mathrm{~kg}$ weight) to the center of the back of the transducer 
The first of these measures requires modification of the transducer under test, is expensive and time consuming, and in general, is not used. (If, however, we determine that there is a problem with a certain type of transducer, we may insist that the customer have the transducer ground and polished before we will calibrate it.) Figure 1 shows three calibrations of a transducer with re-mounting in between. The transducer frequency response curves in Fig. 1 also exhibit the aperture effect which results in nulls in sensitivity. The locations of these nulls are reasonably predicted by the theory for a wave train crossing a circular aperture. The phase response for this transducer (not shown) has phase jumps at the null locations which are also predicted theoretically.

\section{Through-Pulse Calibration System}

The technique of placing the standard transducer at a location symmetrical to that of the unknown transducer cannot be used for the through-pulse calibration method since the input source is located at the top surface of the block and the unknown transducer is located directly below on the bottom surface (epicenter). We may, however, compare the output waveform from the unknown transducer against the theoretical waveform of the thick plate, epicenter solution.

Several through-pulse calibrations have been performed on the NBS Conical Transducer and the results compared with those from surface-pulse calibrations of the same transducer. For such a transducer, which has a small aperture, the results should be comparable. One such comparison is shown in Fig. 2 . Both calibration curves are on the same absolute scale. It is apparent from the figure that there is substantial agreement between the methods, but that the absolute sensitivity indicated by the two calibrations differs by about $2 \mathrm{~dB}$. The source of the discrepancy is being investigated.

\section{AE SOURCE CHARACTERIZATION}

This part of the AE program is directed toward the deconvolution of the $A E$ source in order to determine its character, to improve system calibration, and to improve defect location. The goal of source deconvolution is to remove the effects of the propagating structure and the receiving transducer on the evolved signal from the captured voltage-time waveform, thus revealing the source characteristics using causal analysis. We have shown (1) that for a plate and a capacitive transducer we can determine the actual source function from the received signal using the inverse transform function. In order to do source determination in more complex structures, we must experimentally determine the transfer function and develop general inversion techniques. To carry this out we must generate a repeatable known source which, along with the measured structural response, can be used to determine the impulse response of any complex structure. We also must develop a computational algorithm for inverting the impulse response to obtain the operator which deconvolves the source waveform. This work also provides a basis for system calibration and for minimizing flaw location uncertainty.

\section{Repetitive Short Duration Source}

Comparisons have been made between electrical spark impact sources and pulsed laser sources. The laser source is effective but the spark source has advantages including portability, simplicity, and low cost. We have perfected 
the charging, switching and triggering circuits so that a few-nanosecond highvoltage current pulse can be discharged with very low jitter between electrodes. The resulting simulated $A E$ waveforms in a structure are essentially identical to one another.

The mode of the spark source is controlled by the configuration of the electrodes. We have emphasized the configuration in which the plate (or structure) is one electrode and a second electrode is just above the plate surface. The stress wave thus generated in the structure can be modeled as evolving from local surface plasma heating, the same mechanism as when using a pulsed laser source for radiation heating. Fig. 3 shows detected signals due to sparking onto a 1.5-in. thick plate. Fig. 3a corresponds to the sensor located at the epicenter; Fig. $3 b$ is for the sensor located on the same surface as the source and two thicknesses away from the source. From such experiments we conclude that: the source is extremely reproducible; the detected signals agree well with theoretical predictions for a dipole source; the surface normal motion has a well-defined negative pulse whose arrival is easily detected (a fact that could be exploited for precise measurements of ultrasonic wave speed measurements); the rise time for the stress wave is less than one-half microsecond; and, although the source generates EM signals, these can be easily isolated in the time domain. In order to increase the signal-to-noise ratio of such measurements, a computer program permits signal averaging of 100 repeated source events in less than 10 seconds.

\section{Signal Processing Techniques for Source Characterization}

Signal processing subroutines, for the dedicated minicomputer-based $A E$ signal acquisition and processing system, will be used to deconvolve the $A E$ source function from the received signal in "complex" structures. These signal processing packages include:

- a fast convolution program with one time series in core-memory and one time series on disk

a direct, exact time-domain deconvolution program based on error-free matrix inversion

a program, based on least-squares fit, for finding the coefficient of the inverse filter by solving the Toeplitz normal equations a program for power spectra estimation based upon the maximum entropy method developed by J.P. Burg.

In order to evaluate various approximation techniques for the deconvolution of the digitized signals in the time domain, the epicenter impulse response function and its exact inverse are chosen for comparison (6). Figure 4 compares exact deconvolution with an approximate least-squares deconvolution. Figure $4 a$ is the impulse response function (transfer function of the plate for the sensor at the eipcenter). Figure $4 \mathrm{~b}$ is its exact inverse obtained by direct matrix inversion, which we first reported in 1977. Figure $4 d$ is the displacement at the epicenter corresponding to a step function impact force. Figure $4 \mathrm{c}$ is the convolution of the inverse function (Fig. 4b) and the step function displacement (Fig 4d). As expected, Fig. $4 c$ is a step function. However, results using a least-square approximate deconvolution are quite different. While the approximate inverse function (Fig. 4e) is a reasonably "good" approximation of the exact inverse function (Fig. 4b), the deconvolved step function displacement, Fig. $4 f$ (the convolution of Fig. 4 e and Fig. 4d), is far from a step function. Further examination by convolving the impulse response and 
the approximate inverse reveals that a $\sim 10 \%$ error is present in the result which accumulates for a step-function source. Clearly, the accuracy of the approximate deconvolution is signal dependent. It is expected that if the source signal is cyclic, then the deconvolved result will be much better than for the stepfunction source. Similar comparisons have been made for other types of sources for the epicenter configuration.

\section{MATERIALS EFFECTS AND CRACK CHARACTERIZATION IN STEEL PLATES}

Substantial progress has been made on producing $A E$ in $A 533 B$ steel plates and also on the longer term milestone of developing a multichannel source characterization facility for quantitative measurements of AE sources.

\section{Reproducible AE in A533B Steel Plates}

The generation of reproducible AE in A533B steel plate has been studied using the indentation technique described previously (7). The as-received plate is quite ductile and thus does not readily produce local stress concentrations which could generate AE through rapid, localized load drops. A number of methods for treating the plate to enhance its acoustic "emissivity" were examined. These included indentation following fatigue, severe cold work, and surface modification with an electron beam. In addition, the electron beam and a Nd-YAG laser were used as sources on the material surface. Of the above, only two showed promise: indentation following electron beam surface modification, and pulsed laser excitation of the material.

A systematic study was performed on the effects, in A533B steel, of temper embrittlement augmented by grain coarsening and hydrogen charging. It was found that this method worked quite well and that a combination of grain coarsening at $1100^{\circ} \mathrm{C}$, aging at $500^{\circ} \mathrm{C}$ up to 20 days, and hydrogen charging produced a substantial increase in AE activity. Figure 5 shows the $A E$ count rate and load during indentation of $\mathrm{A} 533 \mathrm{~B}$ steel in the quenched and tempered condition, i.e., prior to the temper at $500^{\circ} \mathrm{C}$ for embrittlement. The emission is rather smal1. Figure 6 shows the AE rate after aging for 10 days at $500^{\circ} \mathrm{C}$. This shows an increase in count rate due to the aging, which is further enhanced by hydrogen charging. For comparison purposes, Fig: 7 shows AE during indentation of hot-rolled 1020 steel and of glass, the latter being one of the most active materials for producing AE. These results demonstrate that by controlling the heat treatment and charging with hydrogen, the relatively quiet as-received $A 533 B$ can be converted to a material that emits at a rate comparable to the most emissive materials. The temper-embrittled condition is one which could occur, to some extent, in an A533B weld region in the field.

Understanding of this phenomenon is further enhanced by a stress analysis of the subsurface-deformed region of the indentation. There is a compressive zone immediately under the indenter which produces a hemispherical region of triaxial tension at one or more diameters away from the indentation. A typical microcrack is shown in Fig. 8. Due to the shape of the tensile field, the crack need not be directly under the indenter. In addition, there is a region of enhanced hydrogen concentration near the surface which may be responsible for microcracks in hydrogen-charged material generally being either surfaceconnected or shallow subsurface types. This leads to the occurrence of cracks at the side, rather than under the indenter as was found previously 
for tool steels that were not hydrogen charged. While the crack is on the order of 200 micrometers in size, this is small relative to the shortest wavelength examined (about 2400 micrometers) so that the crack will appear to be a "point source". In addition, as shown in Fig. 9, many of the cracks were found to be associated with inclusions.

To summarize, a method has been found which is useful for producing $A E$ in A533B steel plates. Through controlling the heat treatment and through additional methods of modifying the material condition, the amount of $A E$ can be controlled while still keeping the material relevant to conditions in the weld region. This method is now applicable for use on larger plates of A533B steel, where signal reproducibility can be checked with the multichannel system.

\section{Multichannel AE System for Plate Experiments}

Another approach being taken in our $A E$ research program is the development of a multichannel $A E$ signal acquisition and processing system to permit more complete deconvolution of the $A E$ stress drop source. An 02 tool steel plate has been hardened by austenitizing and water quenching. Preliminary tests using such a preparation show that multiple, reproducible AE signals can be obtained during indentation. The indentation system is shown in Fig. 10. It consists of a pneumatic loading indenter, a Faraday cage to minize rf noise, and an $X Y$ translation table for positioning the plate specimen.

The multichannel system is diagrammed in Fig. 11. A set of six wide band transducers (the new NBS Conical Transducer) has been fabricated and there are at least six channels of preamplification, antialiasing filters, and amplification up to $100 \mathrm{~dB}$ with a bandwidth in excess of $10 \mathrm{MHz}$, available for the system. Computer codes for the dynamic plate response have been extended to include all surface dipoles, and calculations have been made of emission signals on a stainless steel plate which will be used for additional tests on the wideband transducers. Computer codes for deconvolution, and comprehensive driver programs for our high-speed interface, are being prepared on our minicomputer system which, in turn, is being expanded to carry out data processing.

\section{REFERENCES}

1. D. G. Eitzen, F. R. Breckenridge, R. B. Clough, E. R. Fuller, N. N. Hsu, and J. A. Simmons. "Summary of Fundamental Developments for Quantitative Acoustic Emission Measurements". EPRI Report NP-1877, June 1981.

2. F. R. Breckenridge, C. E. Tschiegg, and M. Greenspan. "Acoustic Emission: Some Applications of Lamb's Problem". Journal of the Acoustical Society of America 57, 1975, pp. 626-631.

3. N. N. Hsu and F. R. Breckenridge. "Characterization and Calibration of Acoustic Emission Sensors." Materials Evaluation 39, 1981, pp. 60-68.

4. F. R. Breckenridge. A paper entitled "Transducer Calibration by Means of the Seismic Surface Pulse." For submission to the Journal of Acoustic Emission. 
5. F. R. Breckenridge. "Surface-Wave Displacement: Absolute Measurements Using a Capacitive Transducer." Journal of the Acoustical Society of America 69, pp. 1177-1185.

6. N. N. Hsu and D. G. Eitzen. "The Inverse Problem of Acoustic Emission -Explicit Determination of the AE Source Time-Function". Presented at the AF/DARPA Review of Quantitative NDE, August 1981, Boulder, CO.

7. R. B. Clough and J. A. Simmons. "Reproducible Acoustic Emission Signatures by Indentation in Steels". Materials Evaluation 39, 1981, pp. 1026-1037. 


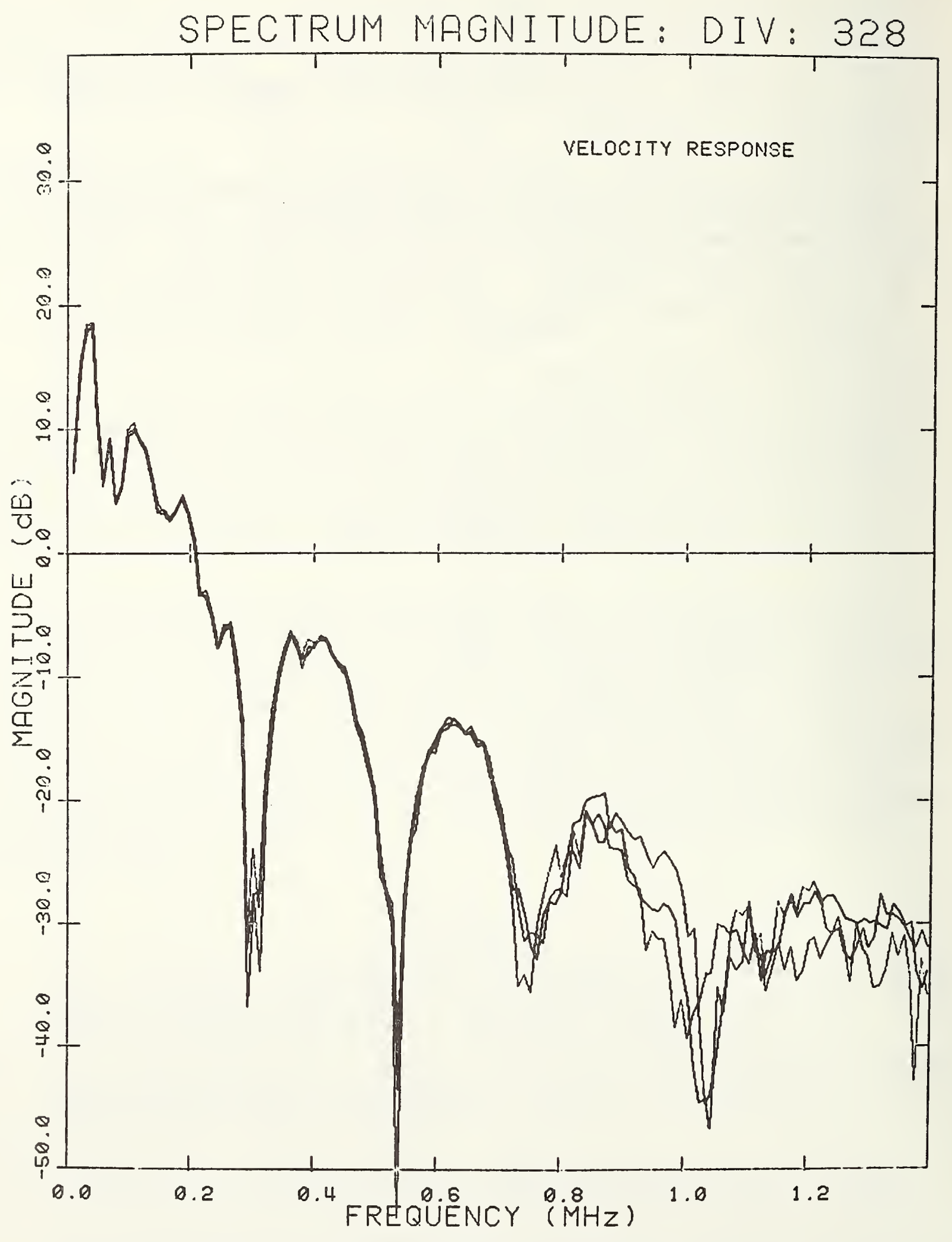

Fig. 1 


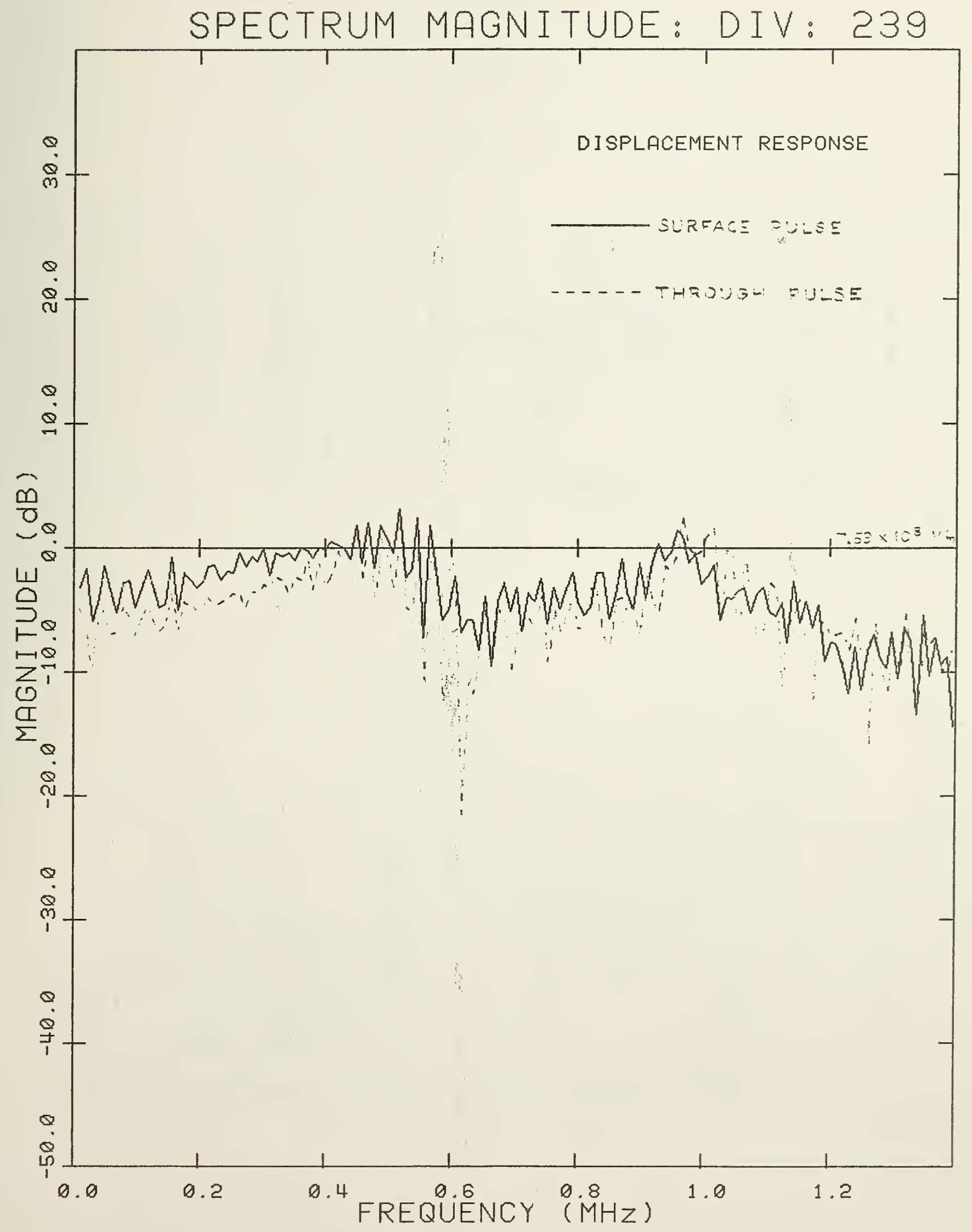

Fig. 2 

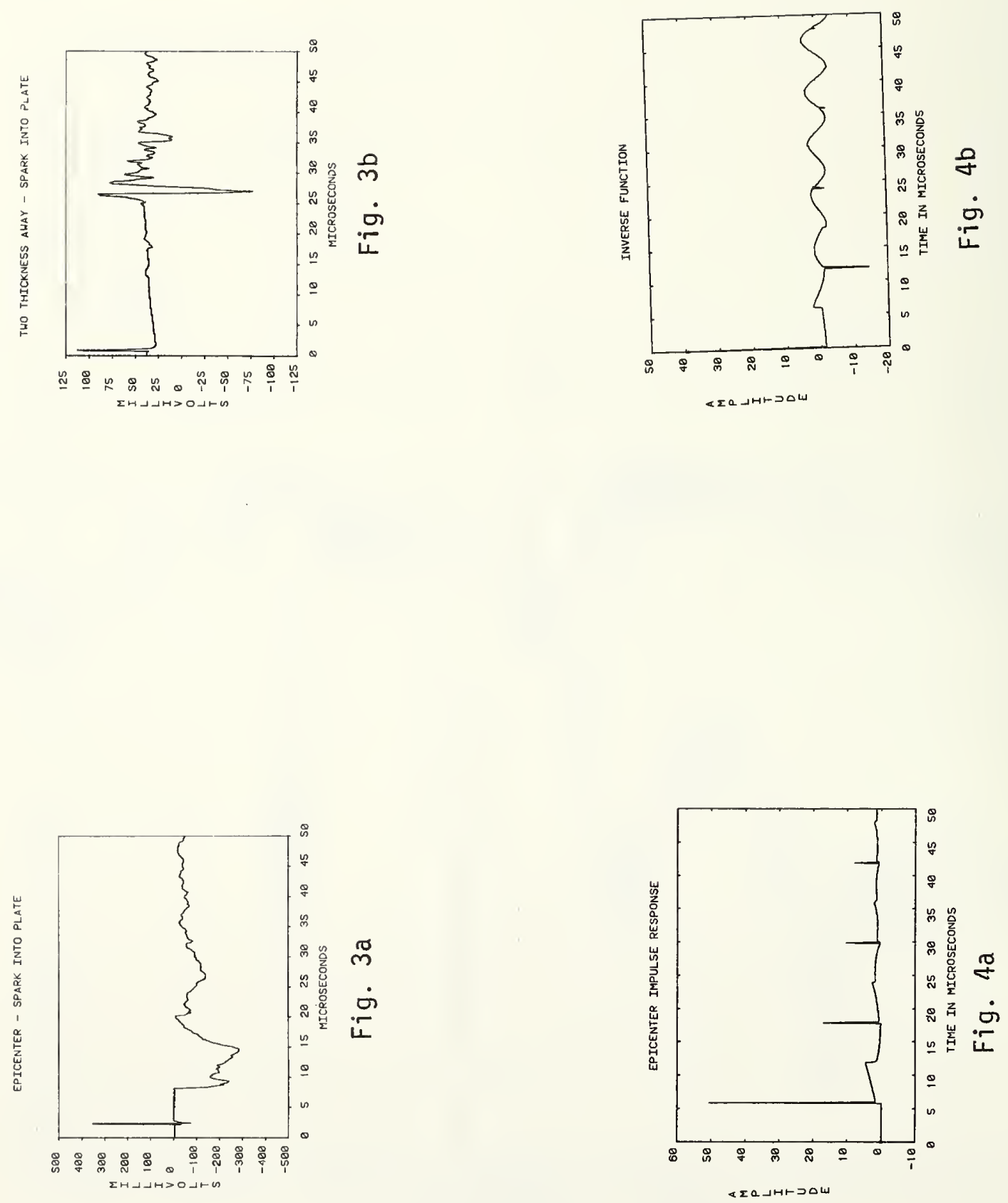

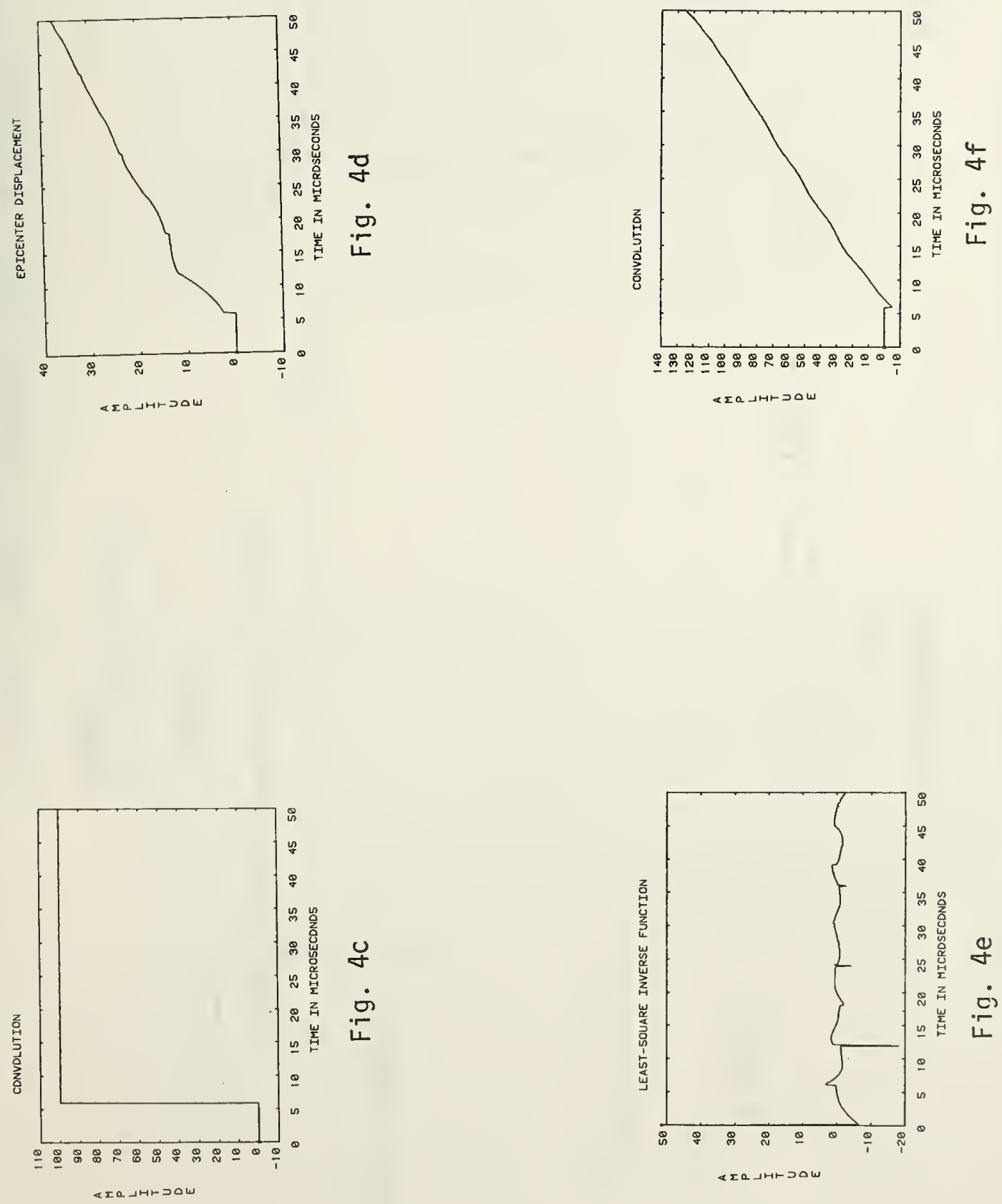

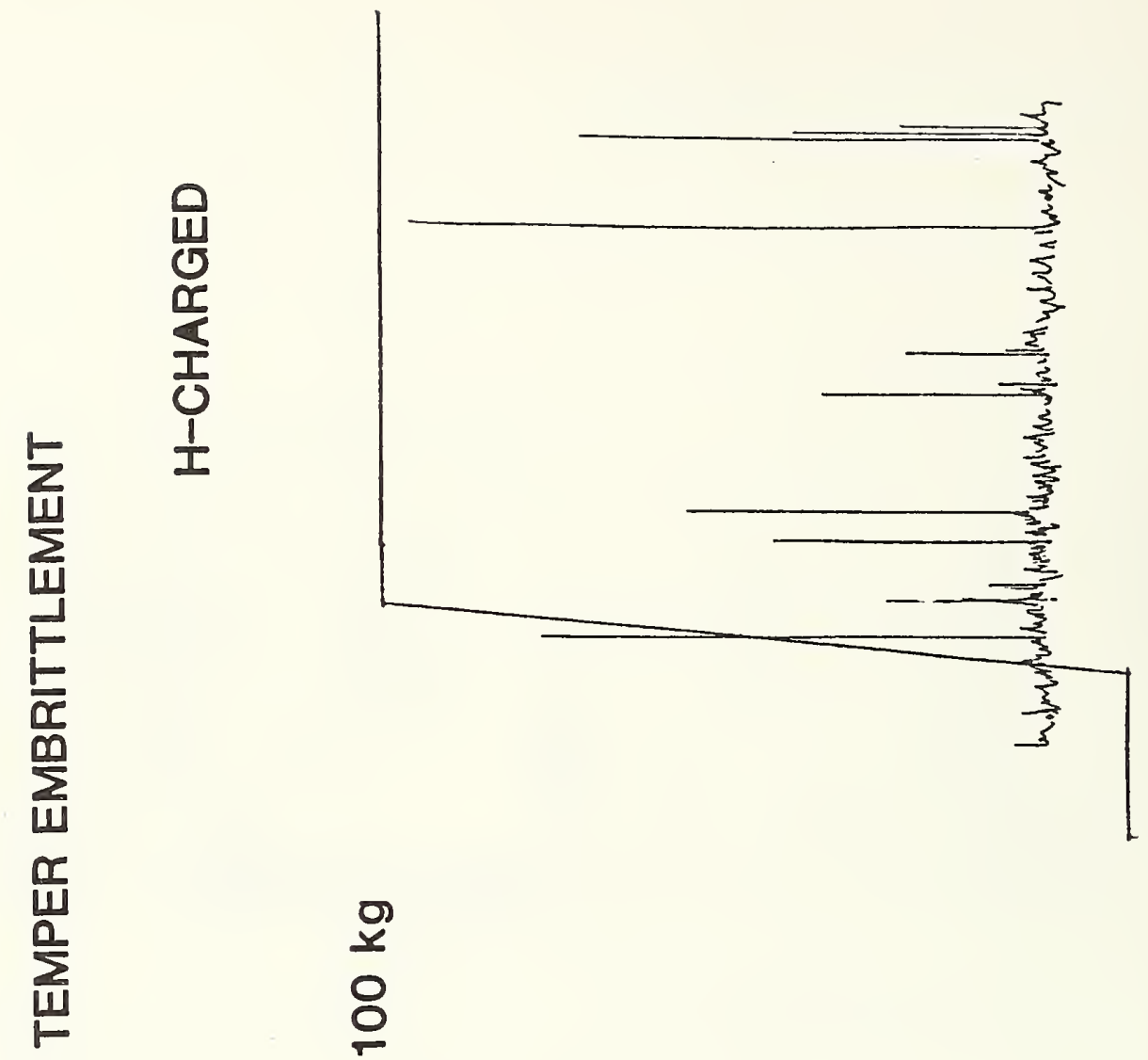

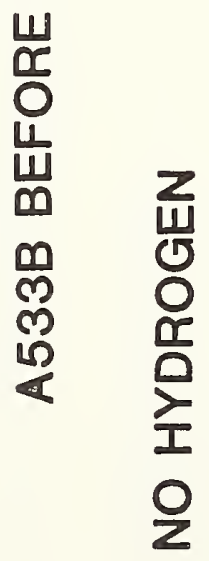

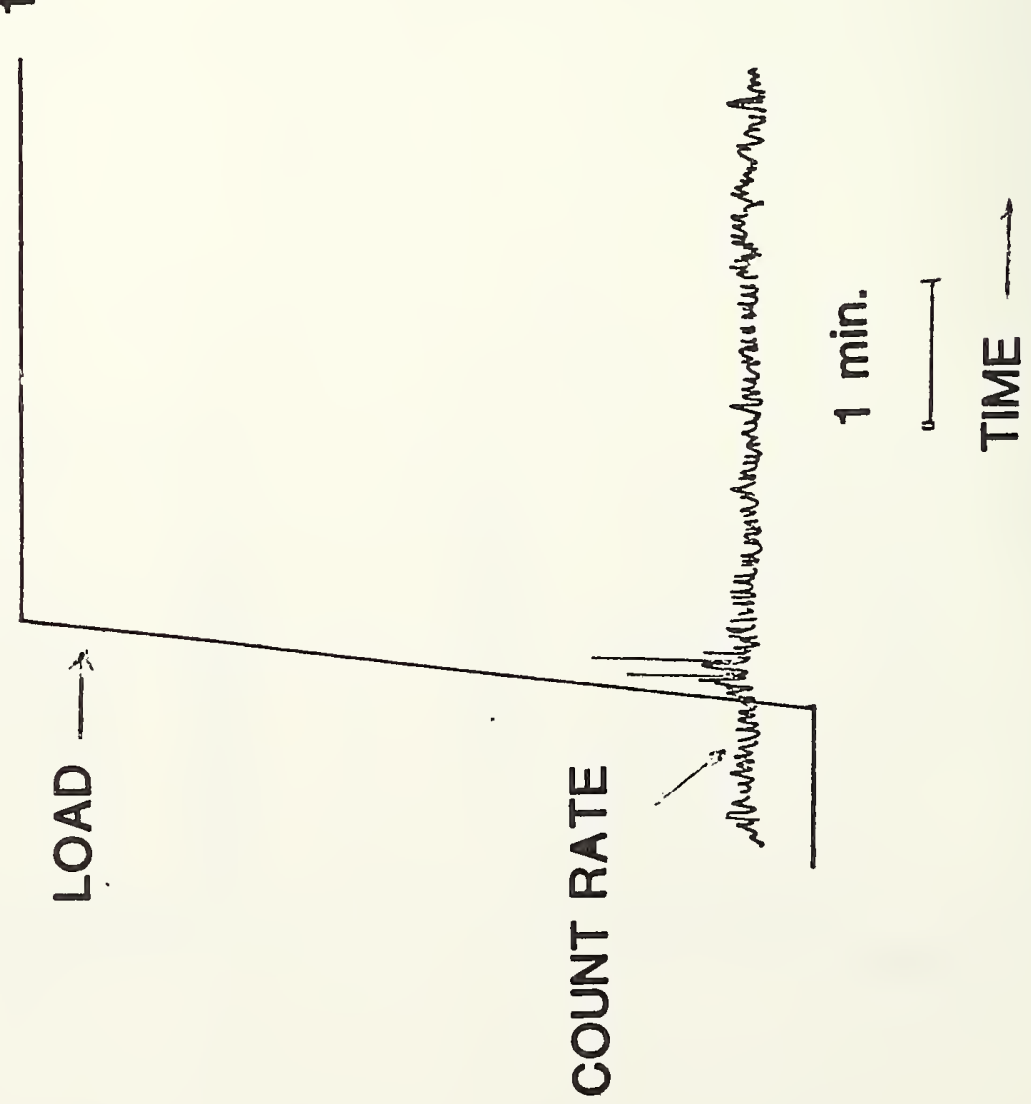




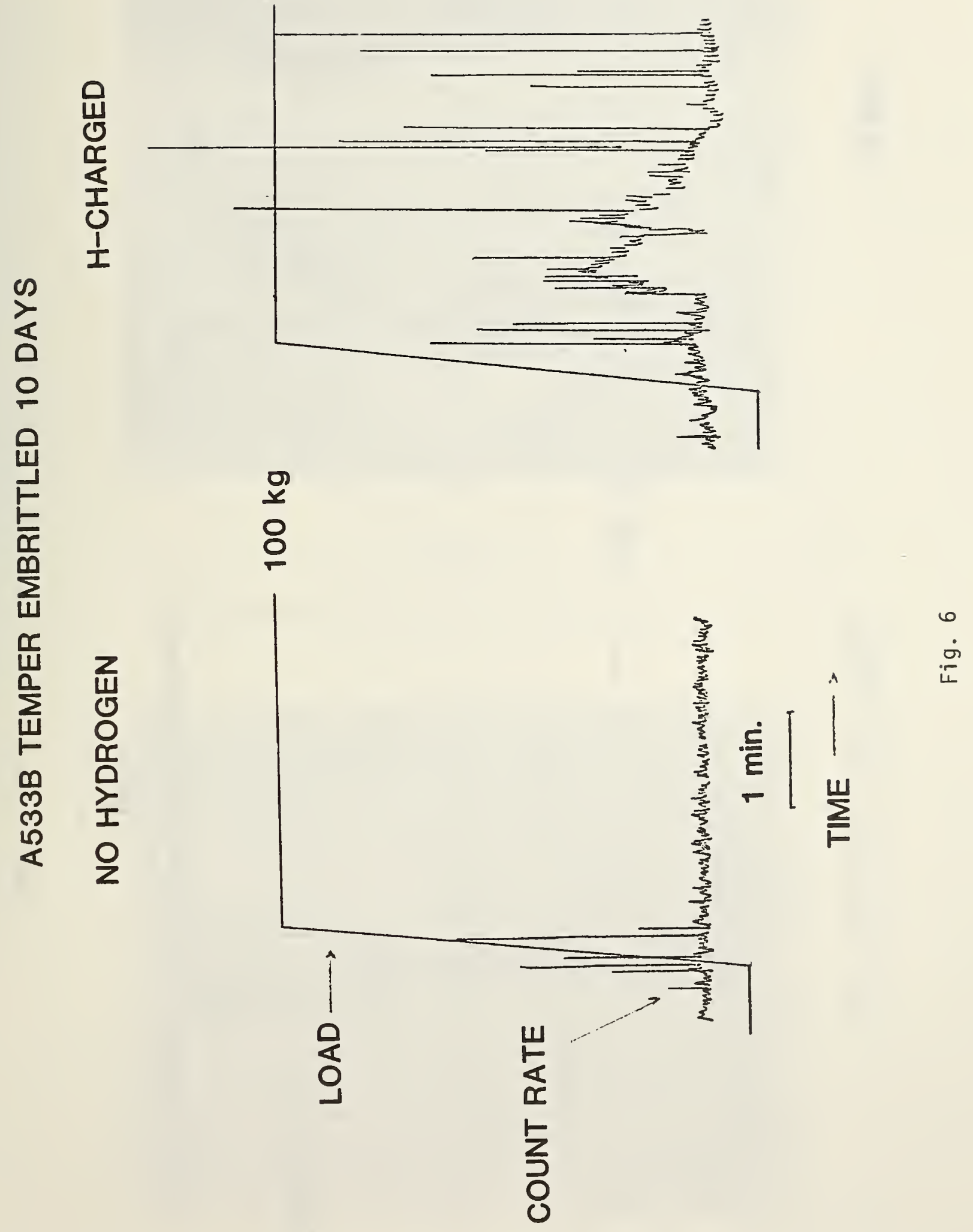




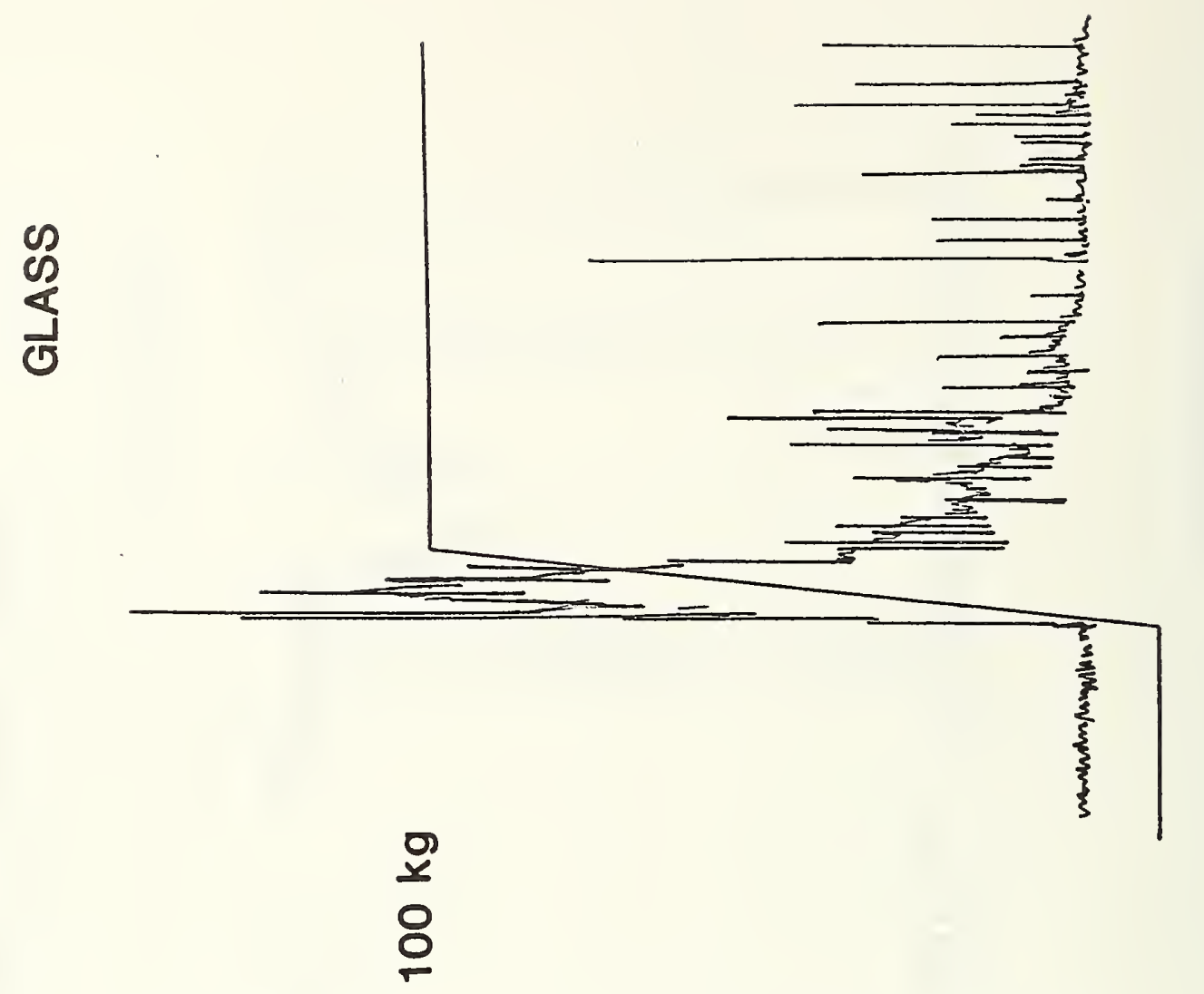

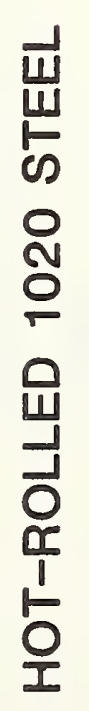

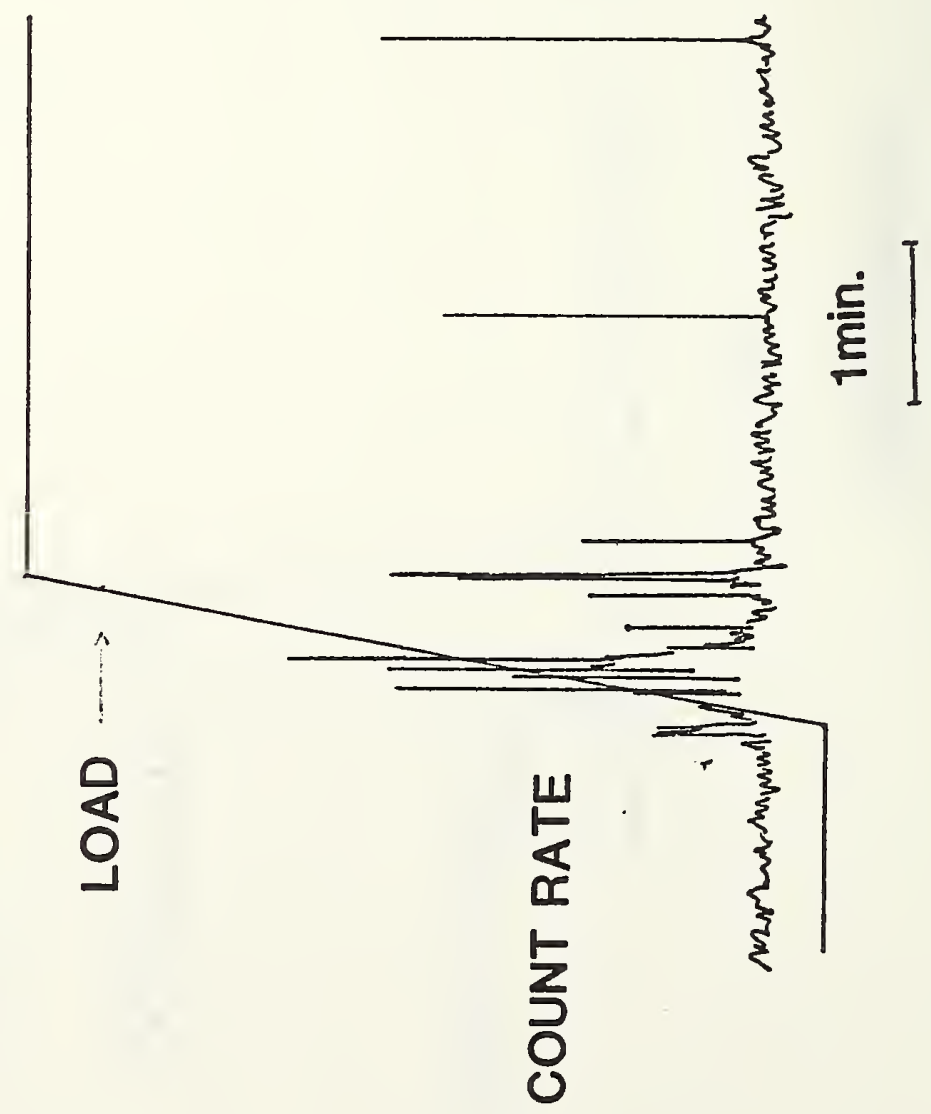




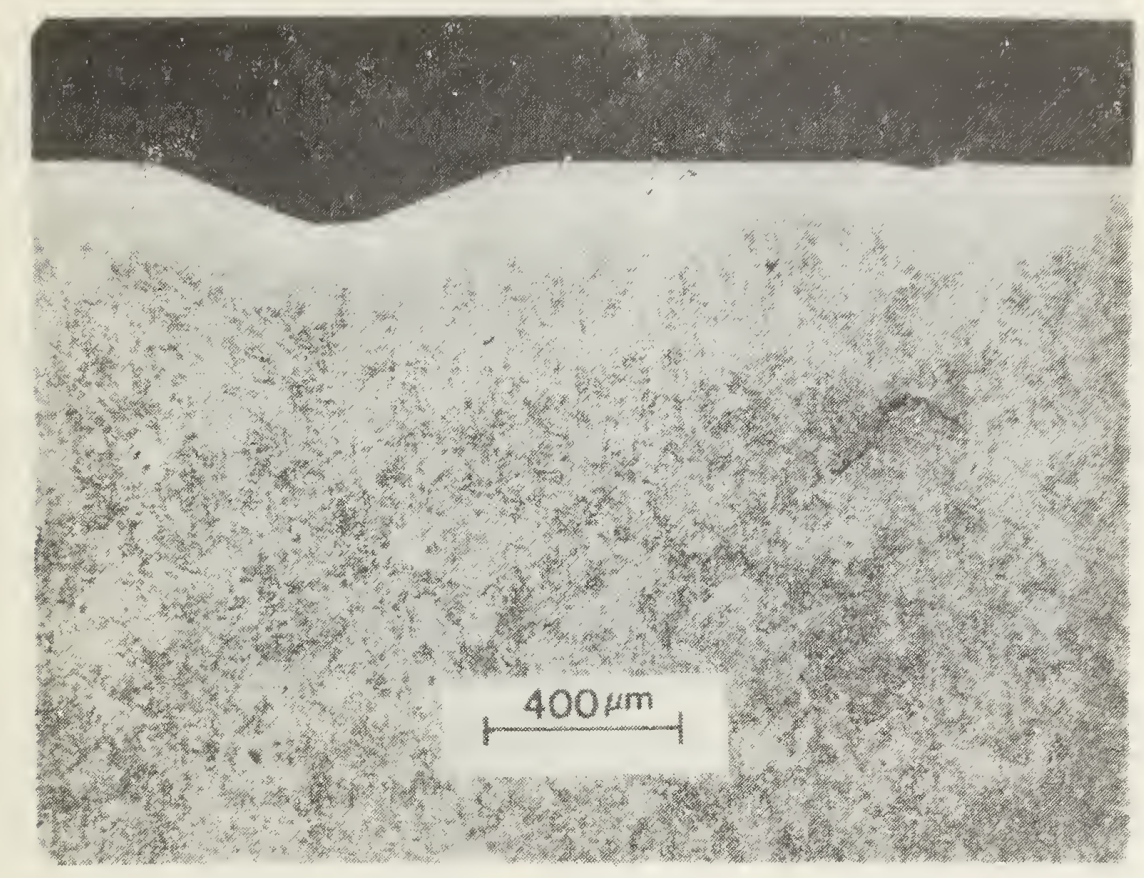

Fig. 8

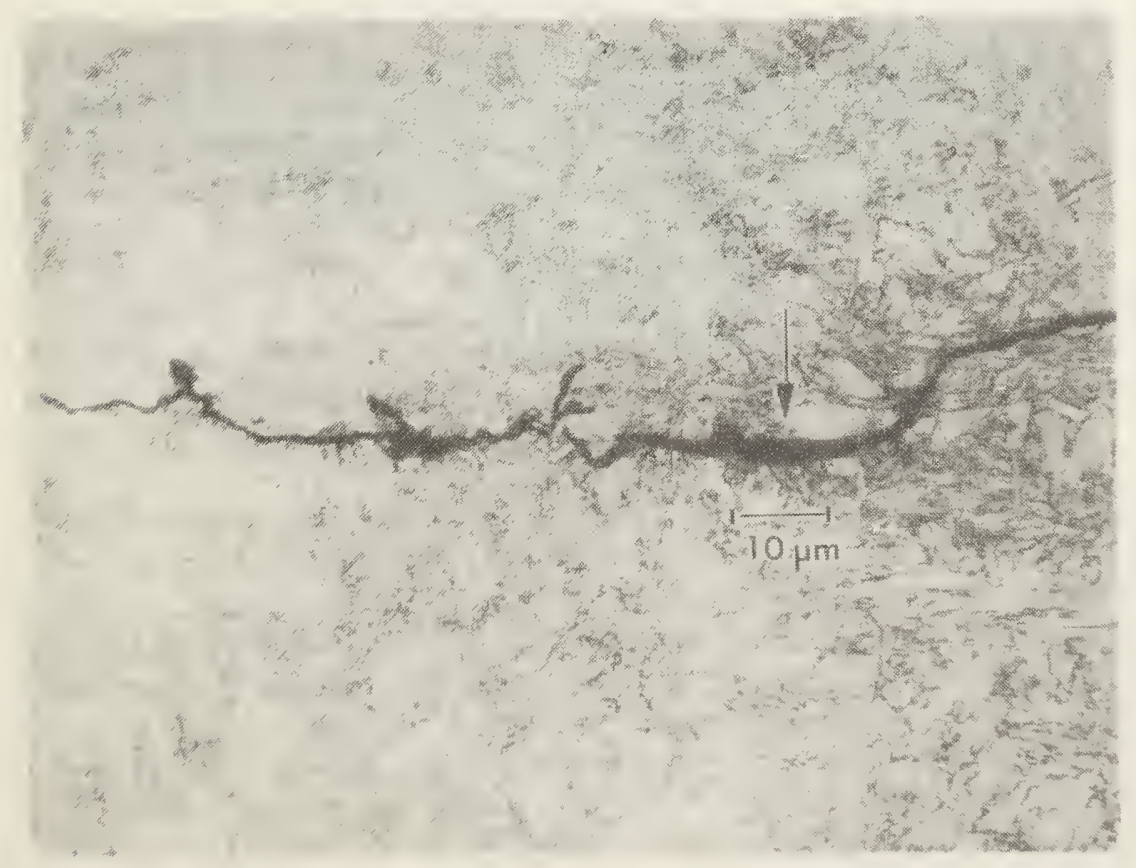

Fig. 9 


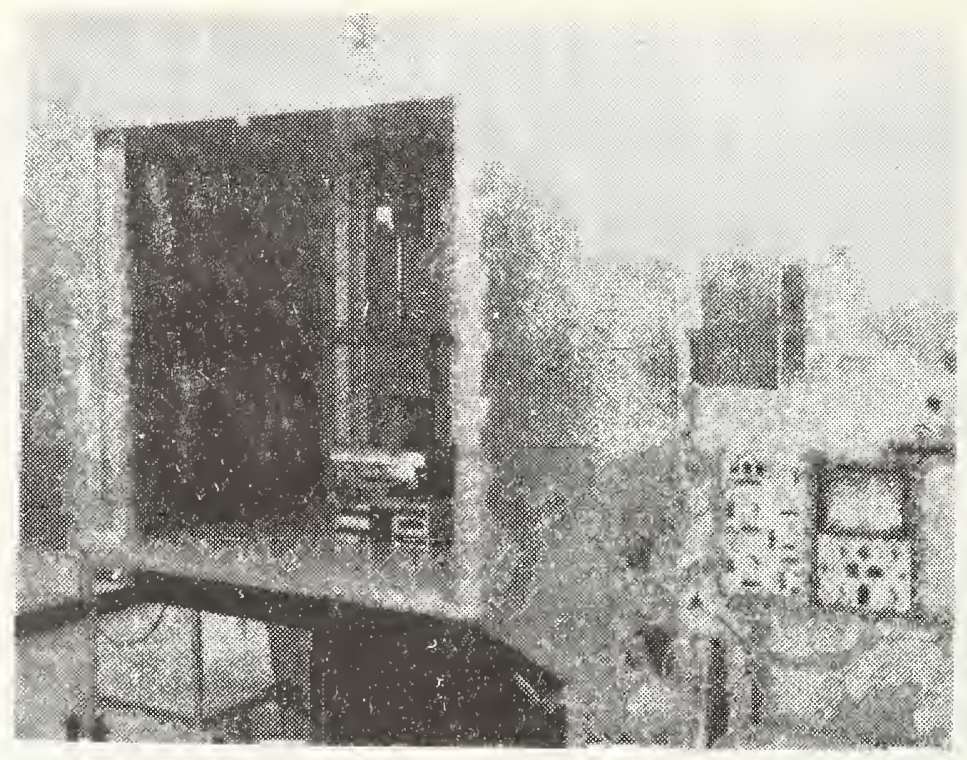

Fig. 10

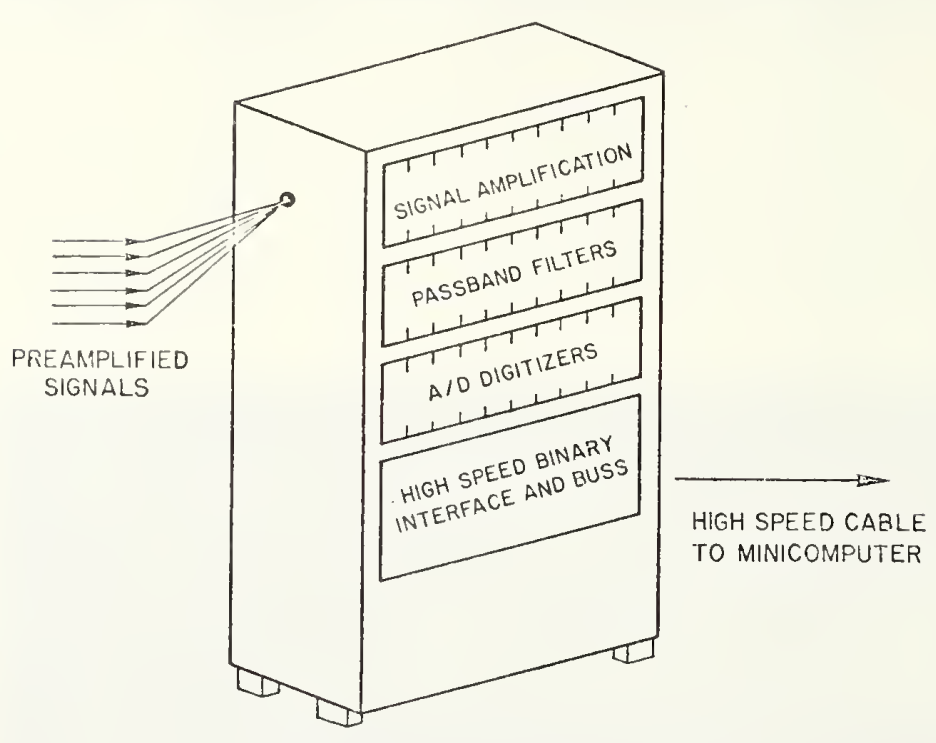

- signal dump and pearm inj $<100 \mathrm{~ms}$

- 00 de UYPAAISIi, RANGE

Fig. 11 


\section{NBS Conical Transducer: A Quantitative Acoustic Emission Sensor}

Thomas M. Proctor

Mechanical Production Metrology Division

Center for Manufacturing Engineering

Initial investigations with regard to new concepts in acoustic emission (AE) transducers have taken place. The capacitance transducer used in the acoustic emission calibration facility is an excellent transducer for standards work. Its flat frequency response over a wide frequency range makes possible faithful reproduction of the mechanical displacement. Such capacitive transducers are, however, of low sensitivity. They are also difficult to use and require a highly polished surface on the mounting structure. Thus, there is a need for a transducer that does not have these limitations and yet has good transient response.

We have constructed such a transducer; it is a simple two-piece device with a conical piezoelectric element of about $1.5 \mathrm{~mm}$ diameter and an extending brass backing. The piezoelectric element has no wear plate and is small enough so that it behaves nearly as a point receiver for AE signals. The brass backing is a cylinder which is large in both dimensions compared to the piezoelectric element. The simplicity of design, small size of the element, and large size of the backing contribute to make it a very high fidelity device with significant improvement over the normal commerciallyavailable piezoelectric transducers. Usually, commercial transducers have severe internal resonances which obscure the received mechanical signals so badly that meaningful quantitative signal analysis is difficult. Certain physical design parameters are the cause of these interfering resonances.

A comparison is demonstrated by Figures 12, 13, and 14 . These figures show the electrical signals from three transducers responding to mechanical surface waves produced by a step force. Figure 12 is the output of the NBS standard capacitance transducer. Figure 13 shows signals produced by a commercially-available AE transducer. This waveform shows the serious problem caused by the transducer's internal resonances. The output should be like that of the standard if it is to be optimally useful for signature identification. Figure 14 is the signal from the piezoelectric transducer constructed in this laboratory. This waveform demonstrates the feasibility of designing better transducers for use in signature identification of transient events.

Another comparison further demonstrates the quantitative potential of the new NBS Conical Transducer. Referring to Figure 15, the upper curve is the theoretical prediction of the normal displacement, at a particular location, of a plate surface due to an AE source. The location for the dynamic displacement is two plate thicknesses away from the source. The assumed source is a point-force step-function. The theory is from Pao, Gajewski and Ceranogla and was independently confirmed by Simmons, Hsu and Willis. The lower trace is the digitized signal from the NBS Conical Transducer which was mounted on a $25.4 \mathrm{~mm}$ thick plate, $50.8 \mathrm{~mm}$ away from a breaking-glass capillary source. The agreement is obviously very striking. Continuing work on this new type of transducer will help to determine its usefulness as a transfer or secondary standard and as a field device for acoustic emission NDE. 


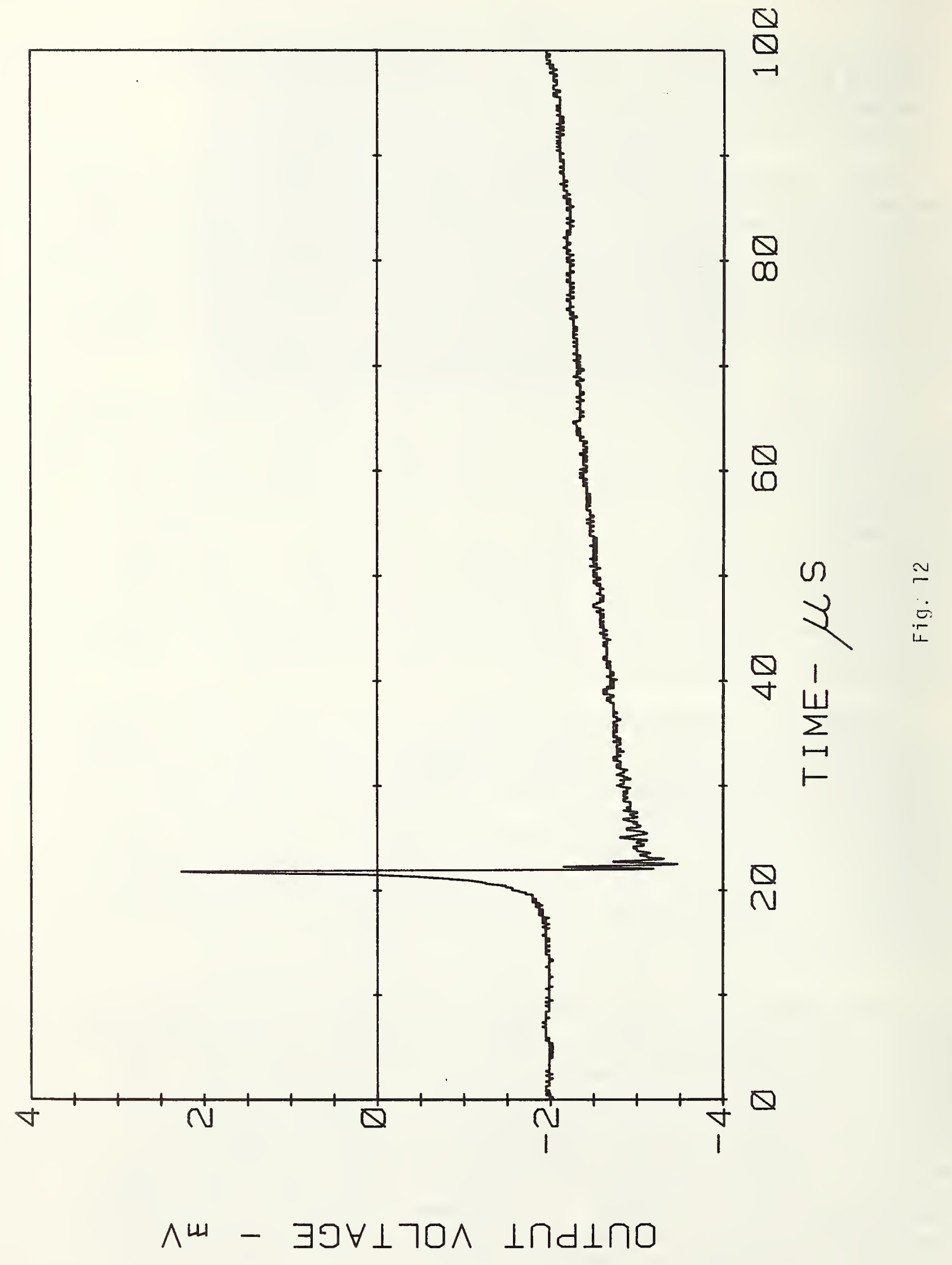




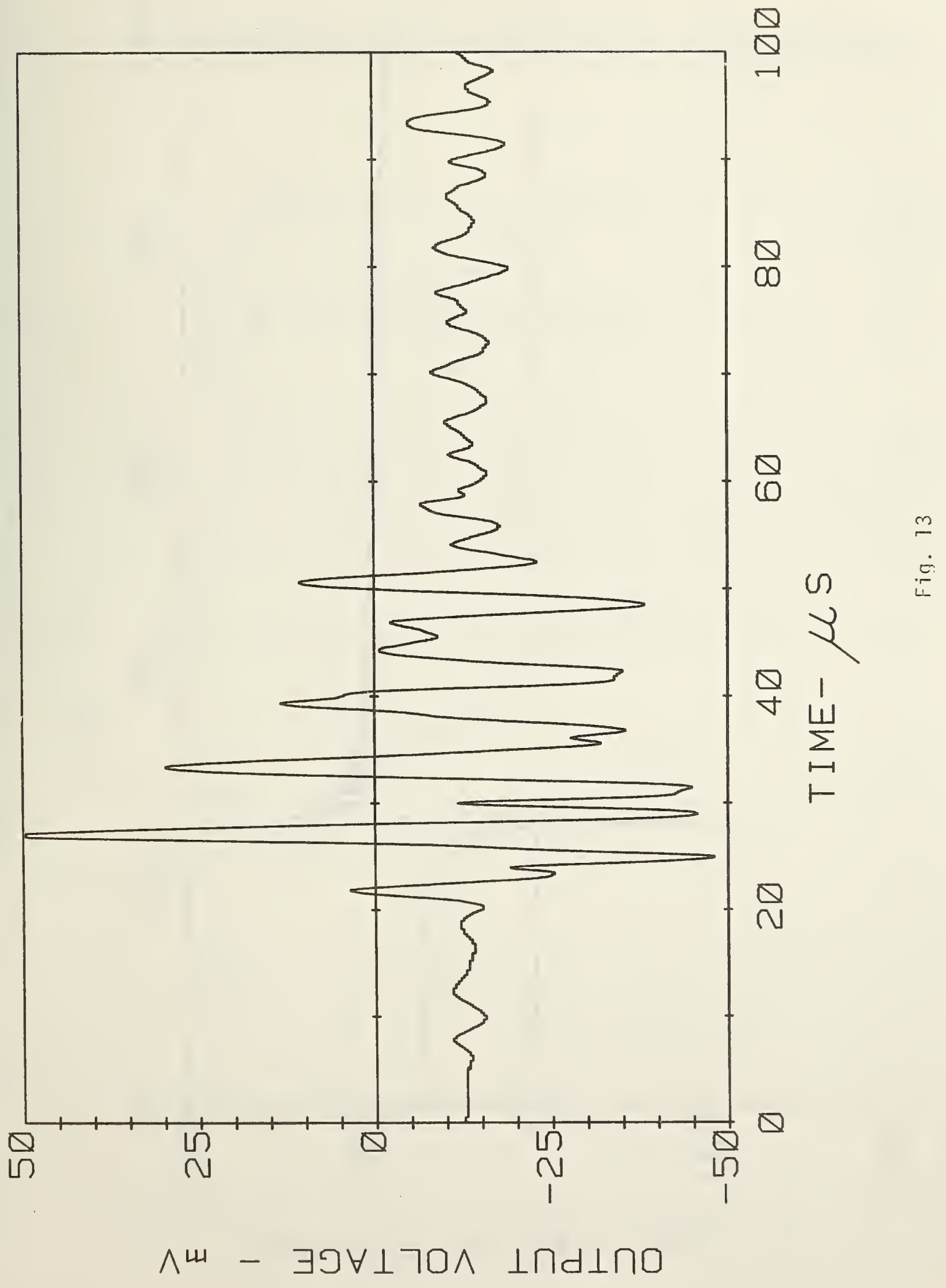




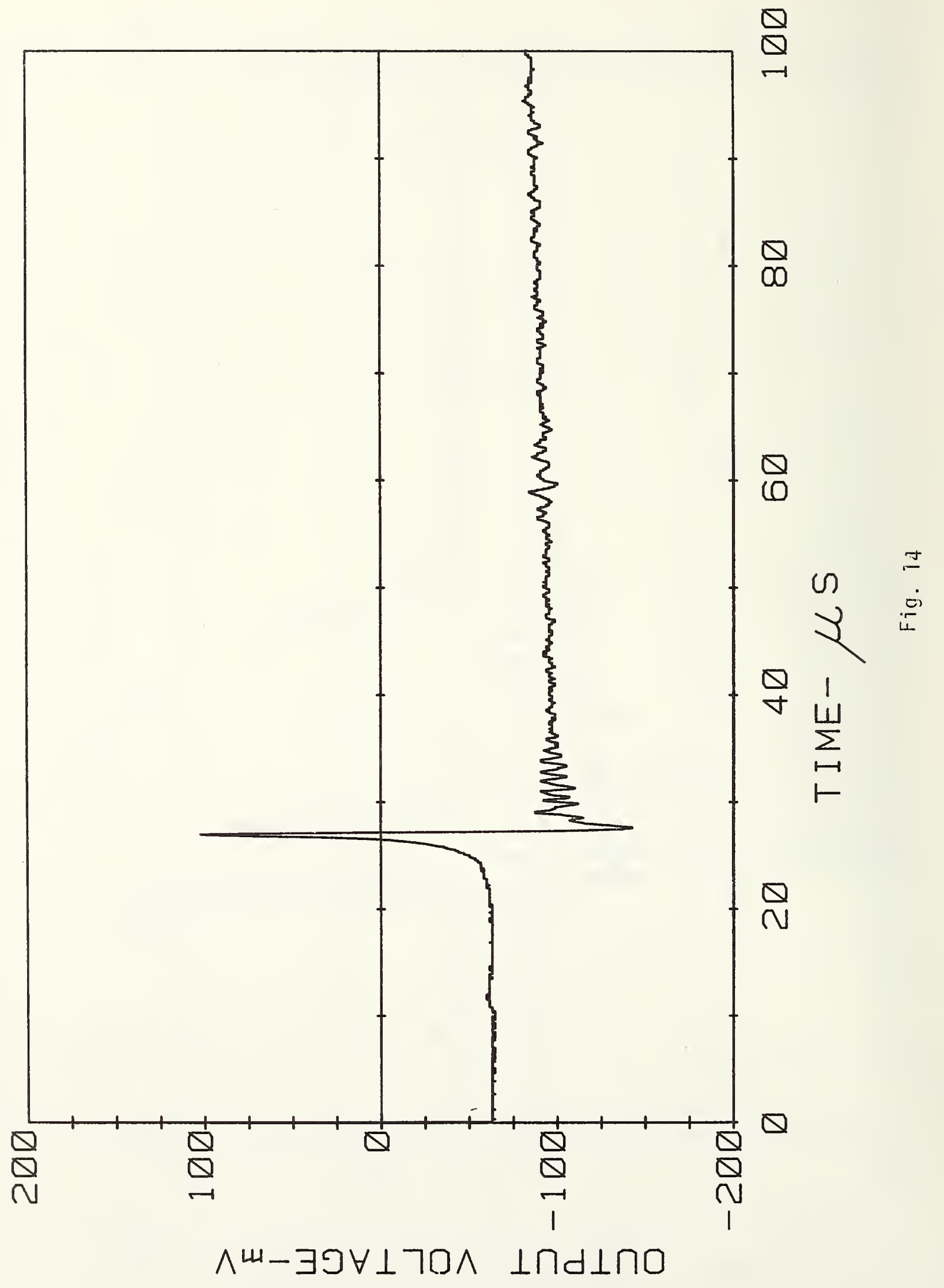




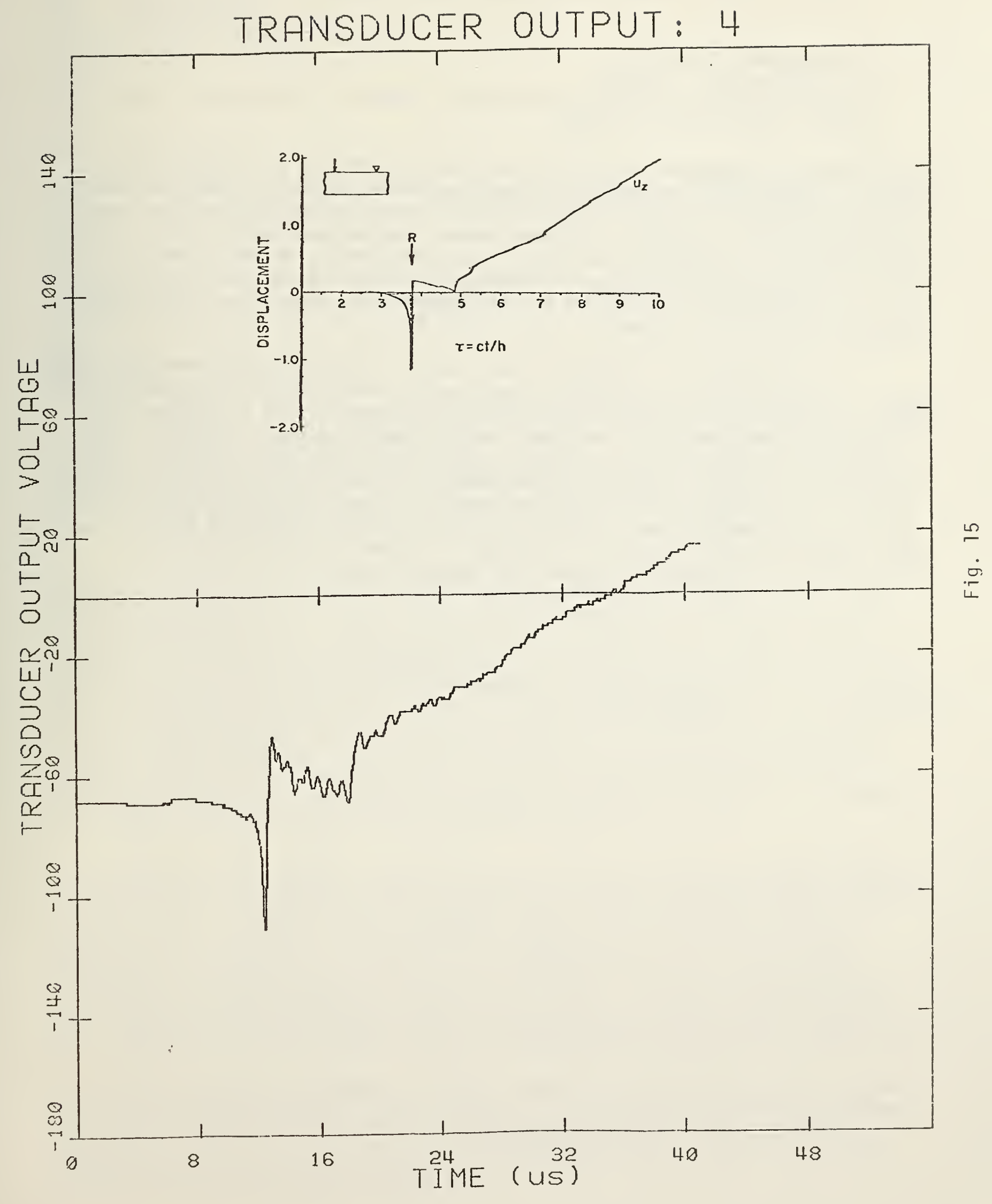


4. Electrochemical Noise

U. Bertocci and J. Kruger

Chemical Stability \& Corrosion Division

Center for Materials Science

The noise measurement apparatus has been interfaced to a computer so that the calculations necessary to separate the deterministic response of the electrode to the input signal from the random fluctuations, as developed and described in a recently published paper*, can be done rapidly and automatically, considerably increasing the productivity of the system. Measurements are now being taken on specimens of nature similar to buried cables to determine if the noise pattern depends significantly and reliably on the corrosive environment.

Noise measurements on buried cables under field conditions have been taken also. Although the instrumentation employed in the field is less sophisticated than that used in the laboratory, limiting the observations to fluctuations in the electrode potential, the use of a two-channel spectrum analyzer, with capability to measure the cross-power spectrum, has enabled us to take some preliminary readings which seem to indicate that the noise signals observed in the frequency range of a few hertz originate from specific portions of the cable. Although these results are insufficient to show that noise measurements can be successfully employed to detect localized corrosion in the field, they do not preclude this possibility.

*U. Bertocci, J. Electrochem. Soc. 128, 520 (1981) 
5. Characterization Kinetics Study of Amorphous Pd-Cu-Si by Ultrasonic Measurements

M. Rosen* and R. Mehrabian

Metrology Division

Center for Materials Science

Metallic glasses exhibit interesting physical and mechanical properties. A glass is generally not in a state of thermodynamic equilibrium and, therefore, tends to structurally relax and ultimately crystallize into a more stable structure when thermal conditions are appropriate. Changes in bulk density and electronic structure associated with the crystallization process are rather insignificant. Nevertheless, appreciable, and in some instances extraordinary, variations in several physical properties were observed. Recently, there has been an increasing interest in the behavior of the elastic properties of metallic glasses in relation with the glass transition and crystallization temperatures.

The objective of the present investigation was to study the crystallization kinetics of $\mathrm{Pd}_{0.775} \mathrm{CH}_{0.06} \mathrm{Si}_{0.165}$ ribbons (about $50 \mu \mathrm{m}$ in thickness) by means of laser-generated and piezoelectrically detected, ultrasonic waves whereby the extensional wave velocity and, consequently, Young's modulus can be determined as a function of crystallization time. The amorphous-to-crystalline kinetics could thus be determined to a high degree of accuracy.

The inherent difficulty of measuring sound-wave velocities in thin ribbons was overcome by developing a technique whereby a load was applied on the specimen by means of rapid deposition of energy from a single-pulse of a Q-switched Nd:YAG laser. A state of unbalanced compressive thermal stress is produced. The stress gradient propagates along the ribbon in the form of an elastic wave. The piezoelectric crystal, at a distance from the spot where the laser radiation was deposited, detects the local disturbance. Thus, the transit time of the propagating wave could accurately be determined by means of a transient-pulse recorder. The complete waveforms, stored in a Perkin-Elmer computer, have not yet been analyzed since they require definition of the theoretical criteria with respect to specimen geometry, conversion of propagation modes and contribution of the detection sensor.

Results of the present investigation indicate that the transition from the amorphous-to-crystalline state in $\mathrm{Pd}-\mathrm{Cu}-\mathrm{Si}$ is time and temperature dependent (Fig. 16). The kinetics were determined at three isothermal-hoiding temperatures. Therefrom, the activation energy for recrystallization was found to be $55 \mathrm{Kcal} / \mathrm{mole}$. From the time dependence of the transformation kinetics an insight was gained into the nucleation and growth mechanisms. Corroborative metallographic evidence was found to be in qualitative agreement with the ultrasonic data (Fig. 17) suggesting a linear growth law whereby the crystallization front moves from the free surfaces toward the interior.

The present investigation has also revealed an initial increase of about $7.4 \%$ in the elastic modulus, out of $40 \%$ for the complete transition from the amorphous to the crystalline state. It appears that this initial increase can be related to a structural relaxation of the amorphous phase that may precede the crystallization process. 


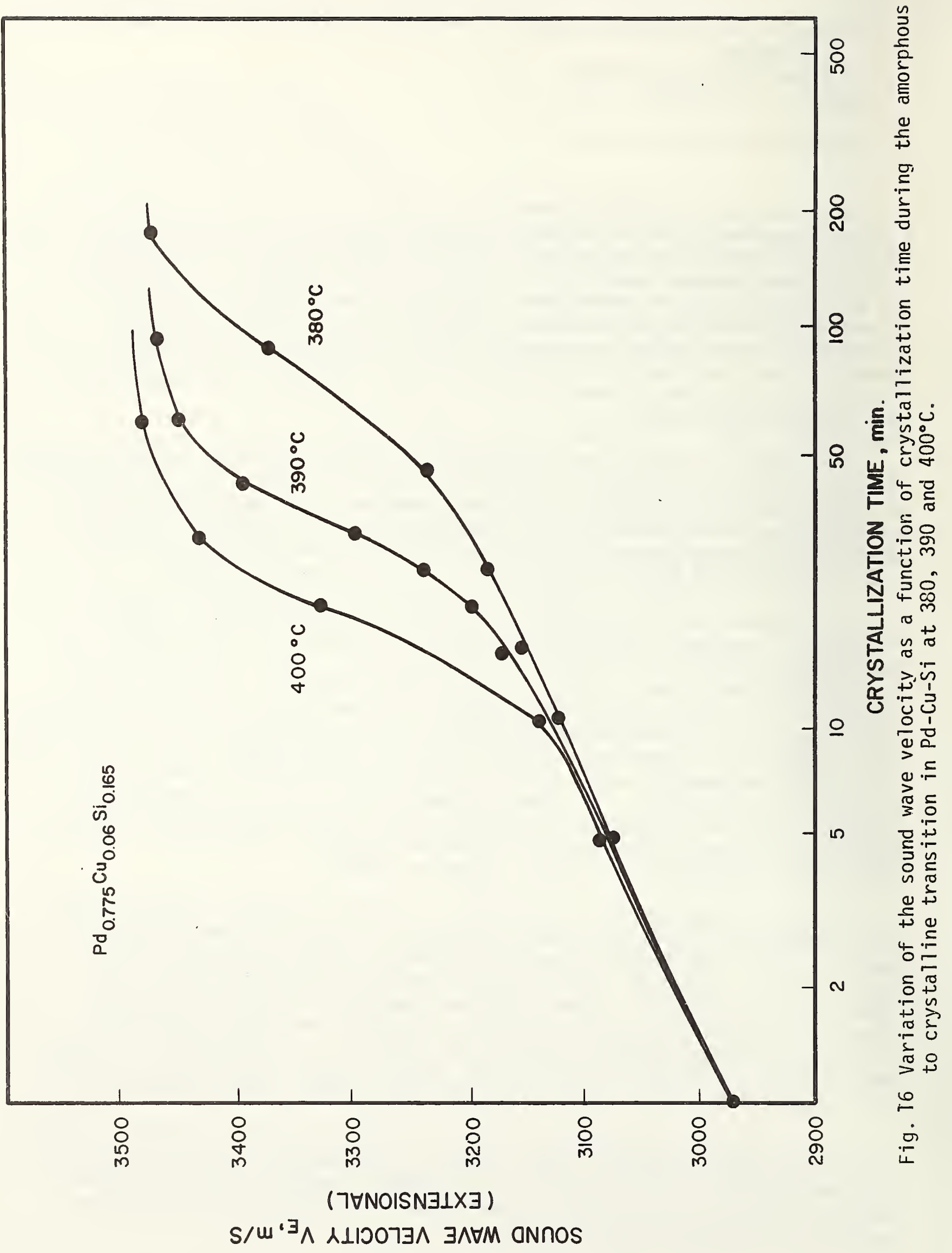




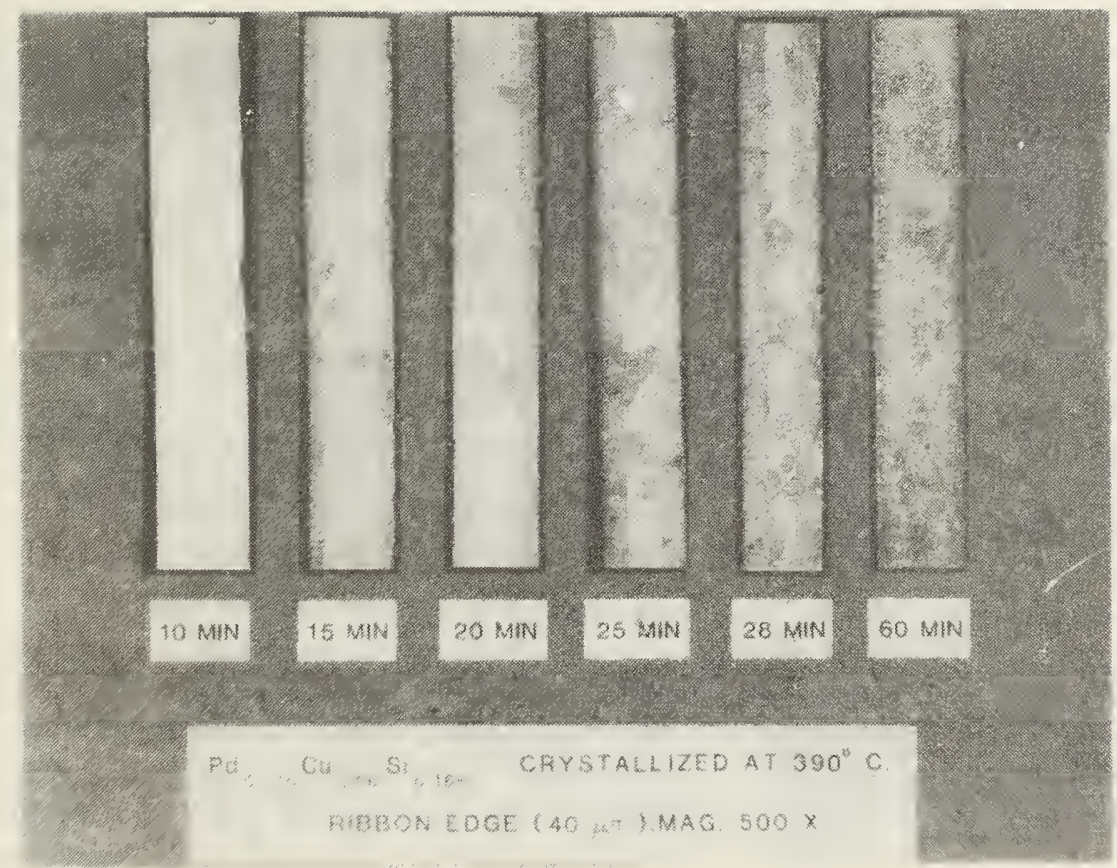

Fig. 17 Changes in microstructure as a function of time during the amorphous-to-crystalline transition in $\mathrm{Pd}-\mathrm{Cu}-\mathrm{Si}$ at $390^{\circ} \mathrm{C}$. View from the ribbon edge. 
6. Nondestructive Evaluation of Composites

H. M. Ledbetter, J. C. Moulder, and S. K. Datta ${ }^{\dagger}$

Fracture and Deformation Division

Center for Materials Science

Detection of low damage levels in composites is a serious, unsolved technological problem; appropriate, reliable nondestructive methods do not exist. Internal friction, which is a gauge of energy dissipation within a material, should be sensitive to this type of damage, particularly at low frequencies where the wavelength is comparable to specimen dimensions. In order to study the effects of small amounts of mechanical damage on the Young's modulus and internal friction of composites, an existing forcedvibration apparatus was modified to permit operation as a freely vibrating reed pendulum. Relative changes in Young's modulus were determined from changes in the specimen's frequency of vibration; internal friction, $Q^{-1}$, was determined by measuring the logarithmic decrement during the free decay of oscillations.

A uniaxial graphite/epoxy composite (HMS/934) was chosen for study. Three specimens $10 \mathrm{~cm}$ long and $2 \mathrm{~cm}$ wide were cut from a $0.85-\mathrm{mm}$ thick panel of graphite/epoxy with the fiber direction oriented parallel to the specimen length. Young's modulus and internal friction were determined by several measurements on each specimen before introducing any damage by mechanical deformation.

Three types of deformation were used to introduce small amounts of damage into the specimens: static tensile loading to approximately 90 percent of the ultimate tensile strength (U.T.S.), tension-tension fatigue at a maximum stress of 60 percent U.T.S. for $10^{5}$ cycles at 20 hertz, and thermal cycling between room temperature and $77 \mathrm{~K}$.

One result observed during the preliminary experiments was that the specimens exhibited a linear dependence of internal friction on strain amplitude at the strain levels used (maximum surface strain $<3 \times 10^{-5}$ ). To remove the influence of strain amplitude on the measured internal friction, data were taken at several different strain levels and extrapolated to zero strain by a linear least-squares fit. Young's modulus, as indicated by the frequency of free vibrations, did not depend on strain amplitude within the precision of the measurements.

The derivative of internal friction with respect to strain, $\alpha$, was observed to change following mechanical deformation, often by an even larger percentage than the zero-strain internal friction. This is illustrated in Fig. 18, which shows the internal friction as a function of strain amplitude for a graphite/ epoxy specimen before and after tensile loading to 90 percent of the U.T.S. As the figure shows, both the zero-strain internal friction and the slope of the strain dependence increase by about 30 percent following mechanical deformation. Young's modulus decreased following deformation by a much smaller amount $(-0.13$ percent $)$.

${ }^{\dagger}$ Consultant to NBS, Department of Mechanical Engineering, University of Colorado, Boulder, Colorado. 
The deformed graphite/epoxy specimen was measured again 24 hours after tensile loading and the changes induced by the deformation were found to have "relaxed." Young's modulus increased to essentially the same value as before deformation. Internal friction decreased from 30 percent to only 11 percent larger than the value for the undeformed specimen. The strain dependence of the internal friction increased over the 24-hour period from 30 to 60 percent greater than the undeformed specimen.

To further substantiate and elucidate these changes in Young's modulus and internal friction with time following deformation, the specimen was loaded to the same stress level and the modulus and internal friction were monitored for several days following deformation. This procedure was repeated three times. The results of one such experiment are shown in Fig. 19, where Young's modulus and internal friction are plotted versus time. All of the results are summarized in Table II, which lists the relative values (referred to the undeformed specimen) of Young's modulus, E, internal friction, $Q^{-1}$, and the derivative of internal friction with respect to strain, $\alpha$, in both the unrelaxed and relaxed states following deformation. In the present coniext, "unrelaxed" refers to measurements taken within 15 minutes of deformation while "relaxed" refers to measurements made at least 95 hours after deformation.

The effect of subsequent mechanical deformation was to produce successively larger decreases in Young's modulus, followed by relaxation to a slightly higher value. Internal friction increased by approximately the same amount after each cycle of deformation, but the component of the increase remaining after relaxation grew larger with successive cycles. The strain coefficient, $\alpha$, showed the largest effect, increasing by more than 200 percent after the second cycle of deformation. The increase in a seemed to saturate after three deformation cycles and the degree of relaxation following deformation became insignificant after four.

The effect of thermal cycling on the Young's modulus and internal friction of graphite/epoxy was just the opposite of tensile loading: modulus increased and internal friction decreased, as Table II indicates. Curiously, the direction in which relaxation proceeded was the same as for tensile loading: modulus increased slightly and the internal friction decreased with time following deformation.

Contrary to expectation from the results on static tensile loading, fatigue induced an increase in Young's modulus of about one percent and a 10 percent decrease in internal friction. Once again, relaxation proceeded in the same direction as for other deformation modes: modulus increasing slightly and internal friction decreasing somewhat more with time following deformation.

The conclusions that emerge from these preliminary studies are both encouraging and sobering: encouraging in that dynamic elastic properties of composites provide a useful tool for studying changes induced by mechanical deformation; but sobering in the complexity of behavior that is revealed. Further studies, both experimental and theoretical, are required to elucidate this complex behavior. 


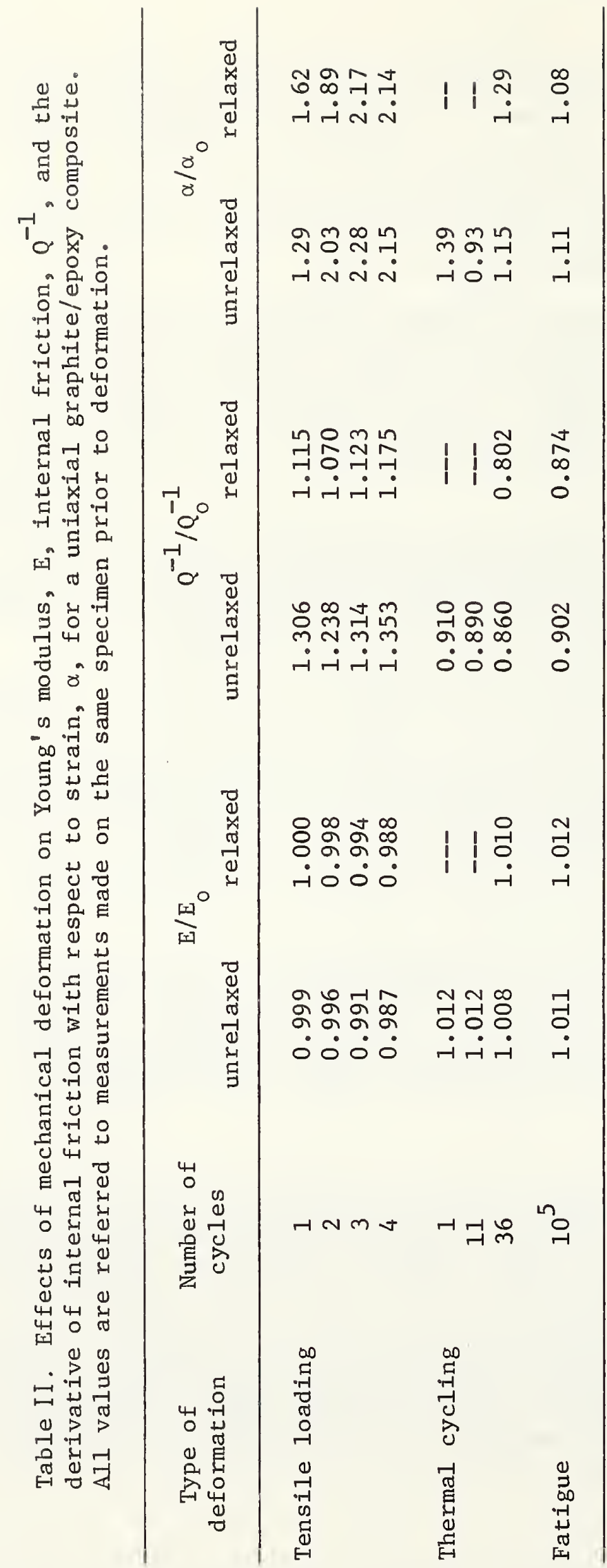




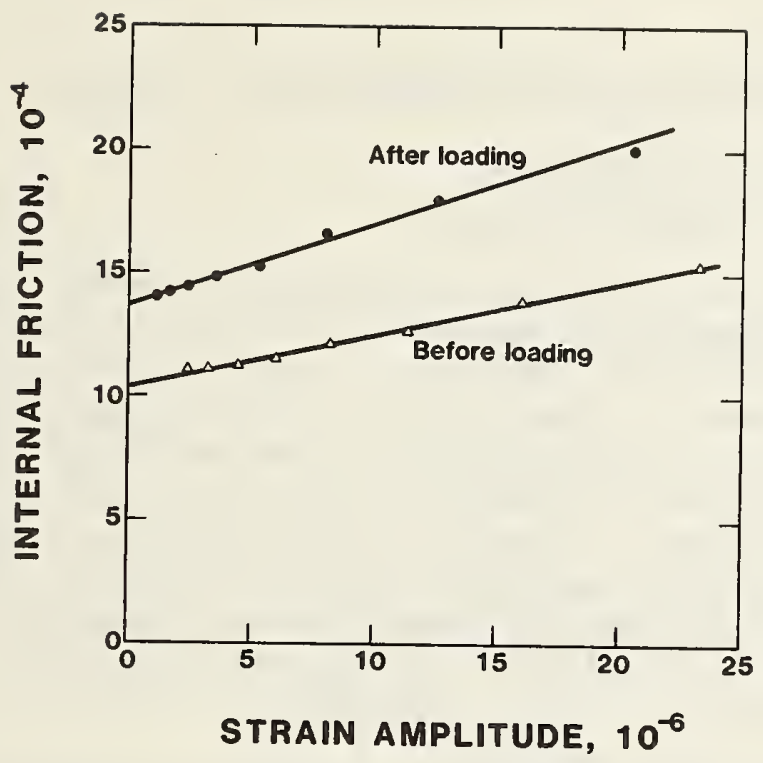

Fig. 18 Internal friction as a function of strain amplitude for a graphiteexopy composite before and after tensile loading to 90 percent of the ultimate tensile strength.

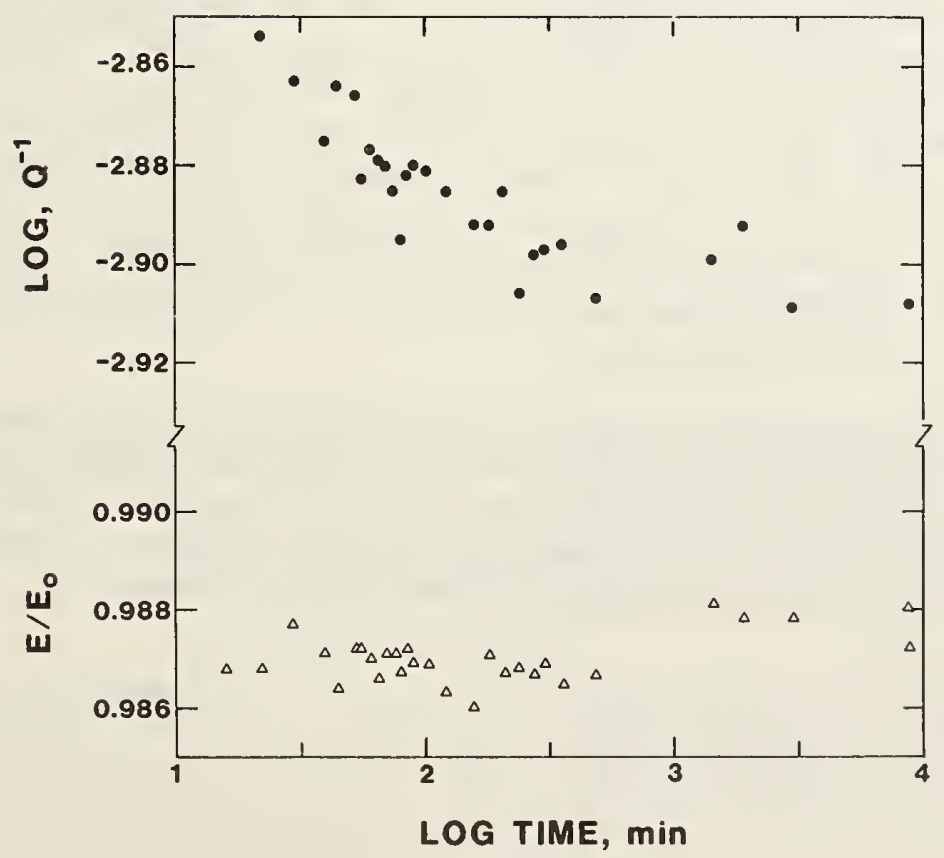

Fig. 19 Internal friction and Young's modulus of graphite/epoxy as a function of time following tensile loading to 90 percent of the ultimate tensile strength. 
7. Application of NDE to Construction/Army

J. R. Clifton and N. Carino

Structures and Materials Division

Center for Building Technology

The use of NDE methods in quality acceptance of installed materials in buildings has been investigated for the U.S. Army Construction Engineering Research Laboratory. At present, when the military has a building constructed, it depends largely on construction inspectors to determine if design specifications and acceptable construction practices are being followed. However, often these inspectors do not have the necessary skills and experience to be effective. Therefore, the military is seeking alternative approaches. An approach being considered is the incorporation of quality acceptance testing of the installed building materials prior to acceptance of the completed building. This quality acceptance testing approach is based upon the use of NDE techniques.

NDE techniques applicable to quality acceptance inspection of commonly used building materials were identified and assessed. Selection of NDE methods were based on considerations of the important properties of the materiais which control their performance and durability. NDE techniques were assessed according to usefulness of information obtained, reliability of the technique, level of expertise required to use the equipment and to interpret the results, and cost effectiveness.

Building materials, components and systems covered in this study are listed in Table III. Their important design factors or properties are also given. Selection of the materials, components and systems was largely based on considerations of the level of their usage; frequency and severity of problems caused by deficiencies in their quality, uniformity, or performance; and the value of using NDE methods to inspect the material, component, or system. For example, NDE inspection is not necessary to determine that a glass window is broken, while the location of reinforcing steel in concrete is more readily accomplished by NDE inspection than by coring. Staff members of the National Bureau of Standards, and of the U.S. Corps of Engineers assisted in the selection process.

The results of the assessment of the NDE methods will be given in the paper "Nondestructive Evaluation Methods for Quality Acceptance of Installed Building Materials", which is being prepared for publication. The report NBSIR 80-2163 "Nondestructive Evaluation Methods for Qual ity Acceptance of Hardened Concrete in Structures", was published January 1981. 
TABLE III. FACTORS CONSIDERED IN QUALITY ACCEPTANCE OF BUILDINGS

\section{MATERIAL OR COMPONENT}

1. Concrete

2. Brick and Mortar Units

3. Wood and Lumber

4. Metals

A. Structural

B. Welds

C. Pipes and Tanks

5. Roofing Systems

6. Paints and Coatings

7. Soils

8. Sealants

9. Thermal Insulation

10. Pipe and Drainage Systems

11. HVAC

12. Building Components

\section{DESIGN FACTORS OR PROPERTIES}

Strength, quality, density, reinforcing steel, cracking.

Integrity, thickness, reinforcing steel, presence of inner grout.

Integrity and general quality, density, moisture content, adhesive bond for laminated wood.

Location, type of metal, cracks, corrosion condition, loose connectors.

Weld defects.

Wa11 thickness, leaks and continuity, corrosion condition.

Composition, moisture content, permeability, slopes, self supporting.

Number of layers, film thickness, integrity, adhesion, quality.

Permeability, proper backfill.

Adhesion.

Thermal performance, location, moisture contents.

Flow rate and pressure, no leaks, presence of dielectric joints, no back flow of water.

Proper flow rate.

Design factors or properties of walls and ceilings, foundation and basement, roof, building envelope and floor. 
8. Monitoring of Segregation Processes in Metals by Electrical Resistivity M. B. Kasen

Fracture and Deformation Division

Center for Materials Science

A nondestructive resistivity technique has been developed for quantitatively following the redistribution of solute during grain growth in dilute copper and aluminum al7oys. Unlike Auger and other techniques based on analysis of fractured surfaces, the resistivity technique provides information on the shift of solute from the interior of the grain boundaries continuously during the segregation process.

The method involves careful monitoring of resistivity changes in the $10^{-10}$ ohm- $\mathrm{cm}$ range during systematic grain growth of recrystallized alloys and a correlation of such changes with changing density of grain boundaries. The influence of segregation on resistivity is determined from the separation between experimentally observed curves relating resistivity to annealing temperature (or grain size) and curves reflecting the predicted resistivity change in the absence of segregation. The latter are calculated from knowledge of the specific electron scattering probability of pure (unsegregated) grain boundaries and from knowledge of the boundary density existing at any point in the experiment. Electrical resistivity is sensitive to segregation levels because removing solute from solid solution removes it as an electron scattering source.

Although, theoretically, any resistivity method capable of detecting changes in the $10^{-10} \mathrm{ohm}-\mathrm{cm}$ range could be used, the experimental procedure uses the eddy current decay method to assess changes in residual resistivity at $4 \mathrm{~K}$. This permits rapid measurement of a series of specimens without the need to attach electrical leads.

A typical result obtained during grain growth annealing of ultrapurity aluminum containing $6 \mathrm{ppm}$ atomic of iron impurity is illustrated in Figure 20 along with the calculated change in the amount of solute out of solid solution, $C_{s}$, during the segregation process. The perturbations observed in the experimental curve (solid dots) provide useful information on the details of the segregation process. For example, the first minimum in the curve is found to correspond to development of a segregate concentration of 2.2-2.6 $\times 10^{14}$ impurity atoms $\mathrm{cm}^{-2}$ of existing boundary, regardless of the type or quantity of impurity. This is associated with attainment of a segregate level equal to full boundary saturation. Analys is of subsequent resistivity perturbations is interpreted as a change in boundary structure reflected in a change in boundary scattering probability.

This work is expected to significantly influence the theory of segregation processes and theories of solute-controlled grain boundary migration rates and to eventually impact on the understanding of the atomistics influencing brittle fracture and stress corrosion behavior. 


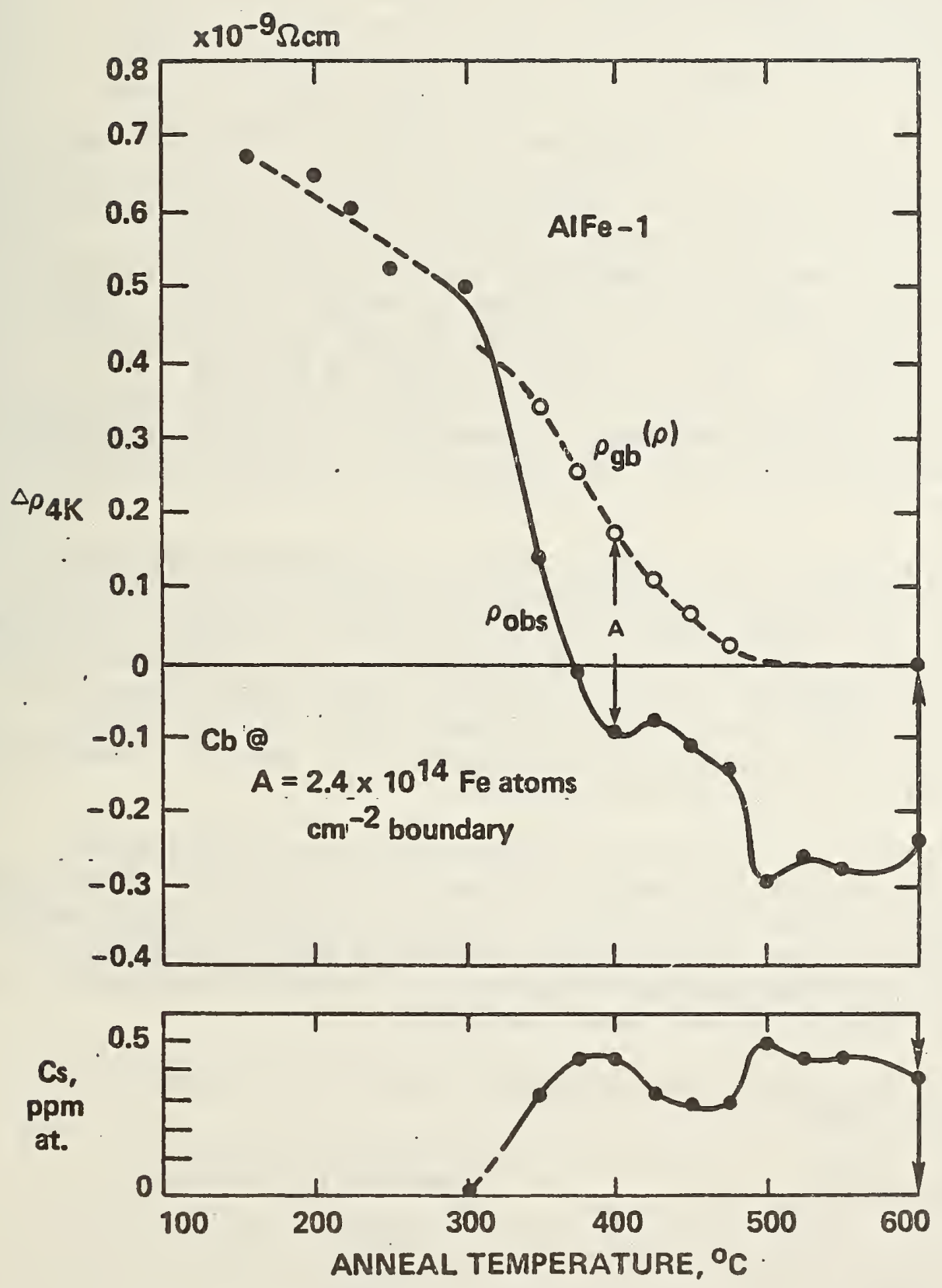

Fig. 20. Experimentaliy observed change in electrical resistivity, $P_{\text {plas }}$,
and the resistivity change predicted from the changing densply and the resistivity change predicted from the changing densple isochronal grain growth annealing of ultrapurity aluminum doped with $6 \mathrm{ppm}$ at. Fe. $C_{S}$ is the concentration of solute removed from solid solution by segregation. Zero on the ordinate scale represents the single crystal resistivity of the material. This resistivity is recovered after extended annealing at the terminal temperature. 


\section{Electrical Conductivity Standards \\ A. J. McAlister \\ Metallurgy Division \\ Center for Materials Science}

The eddy current method is widely used in both the ferrous and the nonferrous metals industries for nondestructive detection of flaws such as cracks, inclusions, and variations in composition and microstructure in metals and metal parts via local changes in electrical conductivity. Primary conductivity standards are needed for reliable execution of this work but, to date, this need has been supplied by industry itself. The necessary range of conductivity for industrial testing is from 0.585 to $58.5 \mathrm{MSm}^{-1}$ (1 to $100 \%$ IAC). NBS is currently acting to provide Al a 1 loy standards in the mid-range of 17 to $35 \mathrm{MSm}^{-1}$ ( 29 to $60 \%$ IACS). One hundred sets of four aluminum samples covering this conductivity range have been supplied to the Electrical Measurement and Standards Division for final calibration.

The properties desired in a primary conductivity standard are longterm stability, durability, and low magnetic susceptibility. (In testing of high susceptibility steels, normal practice has been to apply a saturating magnetic field to reduce the differential permeability to a value near that of vacuum). Hence, elemental metals and alloys of equilibrium phase composition, upon which stable nonconducting surface layers can be formed, are desirable. The surface properties are important not only for durability but for reproducible lift-off behavior as well.

The overall aim is the selection of metals and alloys suitable for primary conductivity reference standards in the 5.85 to 14.6 and 35 to $58.5 \mathrm{MSm}^{-1}$ conductivity ranges, and the preparation of specifications for their preparation and fabrication. Nine candidate materials which appear suitable on the basis of their reported physical properties and probable homogeneity and phase and surface stabilities have been identified.

The following metals and alloys display conductivities within $1 \%$ IACS of the stated values:

$\begin{array}{lc}\text { Material (w/o) } & \text { Conductivity (\% IACS) } \\ \text { OFHC Cu } & 101 \\ 1.3 \mathrm{Zn}+\mathrm{Cu} & 85 \\ 2.4 \mathrm{Zn}+\mathrm{Cu} & 72 \\ 21.5 \mathrm{Zn}, 2.5 \mathrm{Al}+\mathrm{Cu} & 23 \\ 7 \mathrm{Al}+\mathrm{Cu} & 17 \\ 1.5 \mathrm{Si}+\mathrm{Cu} & 12 \\ 3.0 \mathrm{Si}+\mathrm{Cu} & 7 \\ \text { Commercially pure } \mathrm{Ti} & 3.5 \\ 8 \mathrm{Al}, \mathrm{MO}, \mathrm{V} \mathrm{V}+\mathrm{Ti} & 1\end{array}$


For these materials we are investigating the likelihood of attaining the desired metallurgical and surface properties. Questions concerning these materials and their preparation -quality of starting materials, conditions of melting and solidification, heat and mechanical treatment, possible adverse effects in cutting, machining, and surface preparation -are being addressed by search of the literature, and inquiry of industrial practitioners about their experience and methods, and by specific research as necessary. 
10. Eddy Current Standards and Instrumentation

George Free and Bruce Field

Electrical Measurements and Standards Division

Center for Absolute Physical Quantities

Efforts to make available to the general public electrical conductivity Standard Reference Material (SRM) are nearing completion. Two primary standards which will maintain the unit of resistivity have been calibrated, and initial measurements have been made on some SRMs. Four electrical conductivity SRMs will be available in the conductivity range 30\%-60\% International Annealed Copper Standard (IACS).

The experiment determining the resistivity of the two primary standards consists of two types of measurements, length and resistance. The resistivity of a metal bar of rectangular cross-section is calculated using the relation $\rho=(R \times A) / L$. $R$ is the resistance between two points on the bar, $A$ is the cross-sectional area of the bar and $L$ the distance between the two points at which the resistance is measured. Instead of measuring the resistance of the total bar it was measured at a number of points on the bar. All of these points were contained within a $0.12 \mathrm{~m}$ length. The relation $R=R^{\prime}+(a \times L)$ is established, in which $R$ is the resistance measured at specific points, $L$ is the position of the potential contact on the bar, $R^{\prime}$ and a are constants determined by a least squares fit of the data. Since $a=d R / d L$ the resistivity is then $\rho=a \times A$. This is the resistivity at a specific cross section of the bar and not the resistivity over the length of the bar. This calculation permits a more accurate determination of resistivity. Variations in $\rho$ and in the cross-sectional area which take place over the length of the bar are minimized by measuring a portion of the bar which has predetermined uniformity of geometrical and electrical characteristics.

The cross section of the bar (nominal dimensions are $44.4 \times 5.08 \mathrm{~mm}$ was measured as follows: six measurements of the bar thickness and a measurement of the width were done at $25.4 \mathrm{~mm}$ intervals over the total length of the bar. Approximately 200 thickness measurements and 35 width measurements were made. This measurement scheme was repeated four times on each bar to gain some idea of the random error present in the dimensional measurements. The systematic and random errors of the resistivity determinations are being evaluated.

The transfer of the unit of resistivity from the metal bars to the SRMS is done using eddy current instrumentation. The eddy current instrument has been undergoing various refinements to increase sensitivity and make the instrument simpler to use. The present configuration is shown in Fig. 21 The sensitivity to changes in electrical conductivity is about $7 \mathrm{ppm}$. Several problems were resolved during the past year. Lack of correlation was noticed when measurements were made on a set of samples using coils of different geometry. It was found that, although there is negligible coil heating due to the low power levels used, the coil would change resistance value due to 
heat transfer between the coil and the metal sample, i.e., the coil would come to equilibrium at the temperature of the test sample. Temperature coefficient corrections were determined for each coil and the temperature of test samples is determined at the time of measurement.

The eddy current bridge measurements are made by varying the frequency of the source voltage. It was found that any bridge unbalance, i.e., a difference of voltage between the two detector points, caused significant error due to ground capacitance. Thus, the phase discrimination method of eliminating lift-off errors had to be replaced. To make the bridge zero balancing for various amounts of coil lift-off, the coils have been calibrated for changes due to lift-off and a computer algorithm has been written which calculates the correction to frequency necessary to compensate for lift-off and gives a true value of electrical conductivity. These calculations are based on the measured frequency and resistance when bridge balance is obtained.

In the FY 80 report a coil design, Fig.22, using both driving and sensing coils, was discussed. This coil was to be used both for comparison measurements and for an absolute measurement of electrical conductivity. Numerous coils using this arrangement have been made during the past year. The results of tests on these coils show differences between measured values and theoretical predictions of about 1\%. The driving coil was placed directly over the sensing coil. In making the complete coil it was extremely difficult to make surface $(A)$ in the diagram flat. The outer surface of the sensing coils was either convex or concave due to the coil winding process. The difference from the theoretical surface was enough to give the differences that were measured. Due to this error and the precise nature of the planned experiments, it was decided to discontinue this approach to eddy current measurements.

The real-time eddy current flaw detection and characterization system described in FY 80 has required the development of a suitable magnetic field detector. Several types of devices have been investigated, including Hall effect sensors, printed circuit coils and wire-wound coils. The major requirements of any sensor used in the system are resolution of magnetic fields at the microgauss level and "matched performance" between devices. Hall effect devices were found unsuitable due to self-heating of the device, and stray field pickup problems occurred with printed circuit coils. Thus, recent work has involved building matched pairs of wire-wound coils.

The flaw detection system concept relies heavily on using sets (an array) of matched magnetic field sensors. The dynamic range of the signal processing instrumentation requires the sensor outputs to be matched within one percent. We have been unable to produce wire-wound coils with matched responses under the required operating conditions.

Traditional eddy current systems use self-excitation of the coil whereas this system excites the pickup coil using an externally applied field. We have been able to produce coils with nearly identical geometries that are well matched when not in contact with an aluminum plate but produce anomalous results when placed near the plate (i.e., the coils may produce signals of equal magnitude but as much as $180^{\circ}$ out of phase). The degree of mismatch is 
a function of magnetic field orientation, frequency and thickness of the aluminum plate. Fig. 23 shows the magnitude of the difference of two coils when excited in an externally applied, horizontally oriented field separated from any metal (air), and the difference when the coils are placed on an aluminum plate and excited with either a horizontal or a vertical magnetic field.

Consultations have failed to provide any answers to why the coils are mismatched when placed on a metal plate. Until this problem can be resolved we are not able to make any significant progress on the flaw detection and characterization system.

Further investigation of coil geometries will have several objectives. of primary importance will be improvement in 1 ift-off discrimination and reduction of coil resistance change due to temperature variations. Finally, several new approaches to the absolute measurement of electrical conductivity using eddy currents will have initial testing to determine if further investigations are warranted.

Calibration of conductivity standards and of tensile specimens have been done throughout the year to support the Metallurgy Division in its project on the properties of various aluminum alloys. Conductivity measurements were also made for Dr. C. V. Dodd of Oak Ridge National Laboratories. An absolute determination of electrical conductivity using eddy currents is being done at Oak Ridge and NBS examined the test pieces for uniformity of electrical properties over front and back surfaces and measured the difference in resistivity between the samples.

During FY 82 several projects are planned. Two more metal bars will be measured by dc methods. The resistivity of these bars will be greater than that of the present primary standards. Intercomparison of the bars using eddy current methods will serve as a check on transfer techniques and should decrease the total uncertainty of the dc measurement.

Work will be started on the manufacture of SRMs in the range $60-100 \%$ IACS. This will be part of the continuing effort to have SRMs available throughout the commonly used conductivity range. 


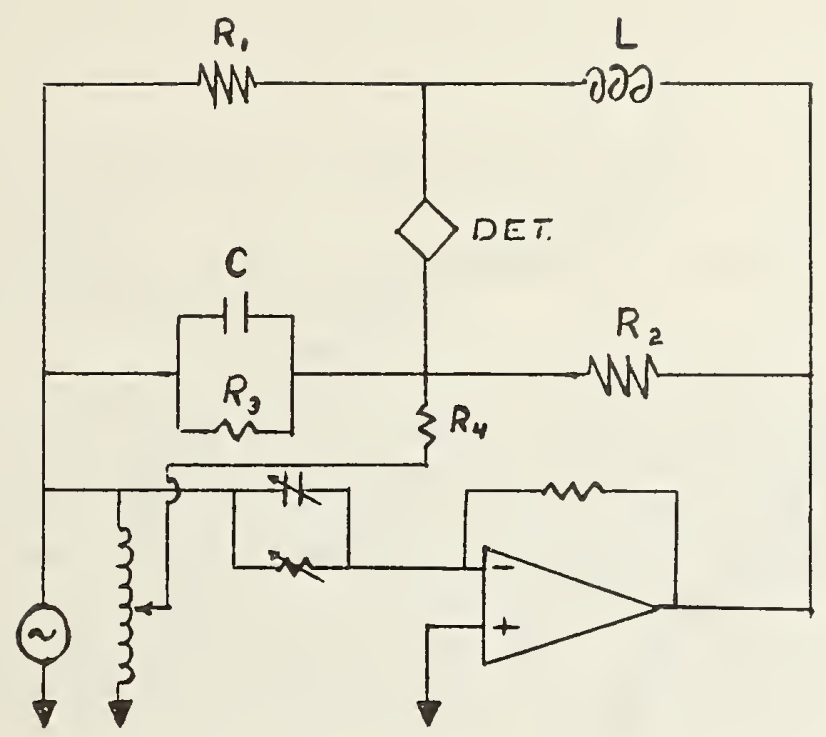

Fig. 21

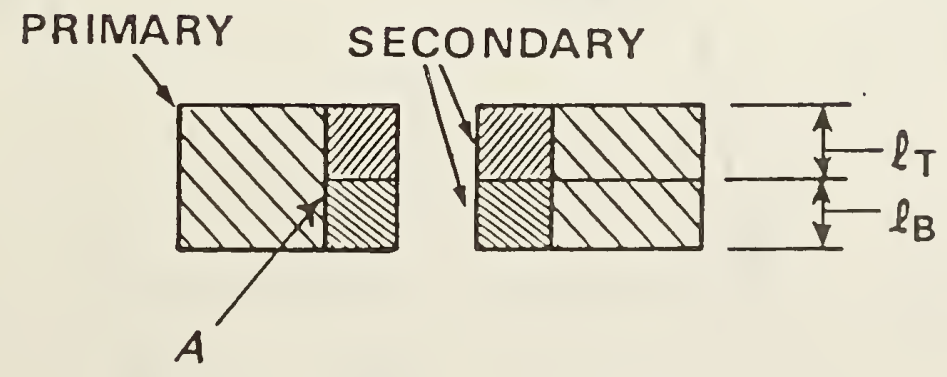

Fig. 22 


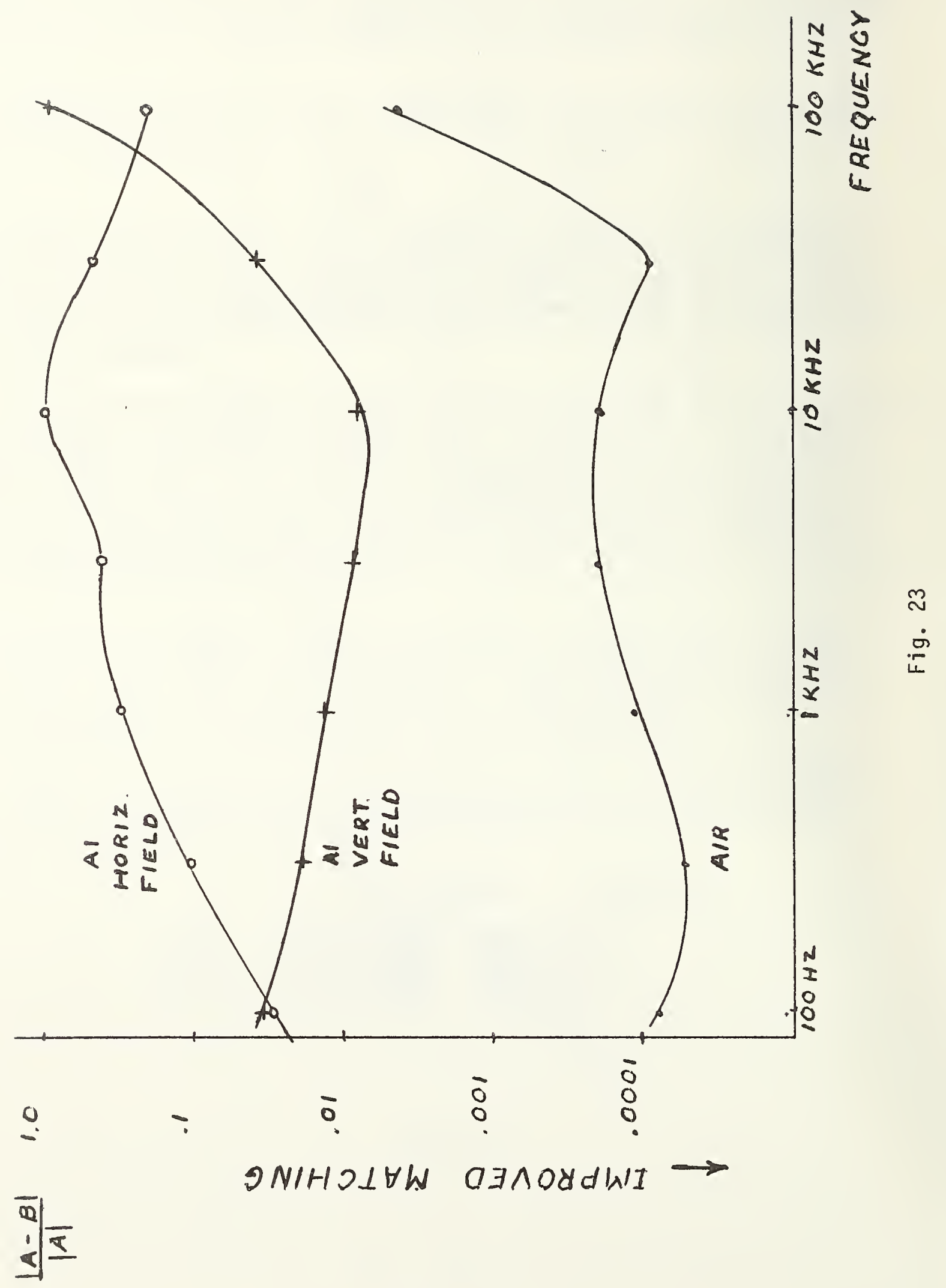


11. Theoretical Modelling in Eddy Current NDE Arnold H. Kahn

Ceramics, Glass and Solid State Science Division

Center for Materials Science

Calculations have been performed of the eddy current density in the vicinity of a crack in a plane slab of conducting material. This work extends previous research performed here, and outside NBS, in covering the cases of arbitrary depth and angle of inclination of the crack with respect to the slab. The calculations were performed by conversion of the eddy current partial differential equation to a boundary integral equation for which numerical methods could be applied. The results are in good agreement with microwave experiments performed at Stanford University, and provide insight into the effects contributing to eddy current signals.

The results of a typical computation are shown in Fig. 24, which is for a perpendicular crack. The circles indicate the real part of the calculated surface impedance and the triangles indicate the imaginary part. The distance $X$ is measured along the slab, and the distance $Y$ along the crack. In this particular case, the depth of the crack is four skin depths. Calculations like this have been performed for a variety of depths and angles of inclination. The technique has given a wide range of results at low cost.

The total impedance change due to a crack is found by integrating the curves of Fig. 24. The dependence of this change on crack depth is shown in Fig. 25 for a perpendicular crack and in Fig. 26 for a crack inclined at $45^{\circ}$ to the surface. 
PLANE SURFACE

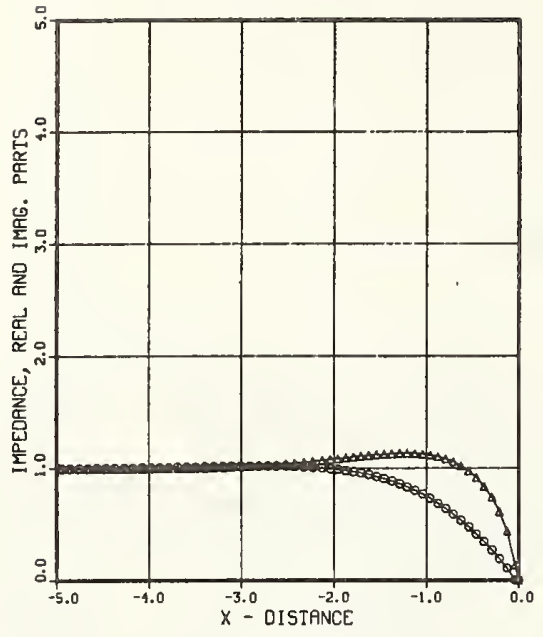

CRACK

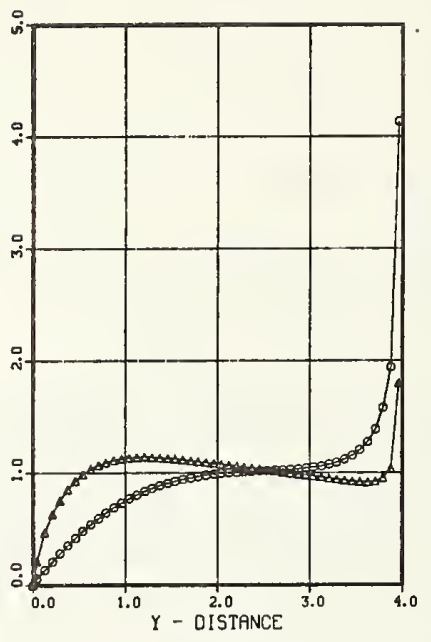

Fig. 24. Normalized surface impedance as a function of position in the vicinity of a perpendicular crack. The real part is marked by circles and the imaginary part by triangles. The crack depth for this case is four times the skin depth. The normalization is such that the curves approach unity far from the crack on the surface.

Normalized Impedance Change

vs.

Crack Depth

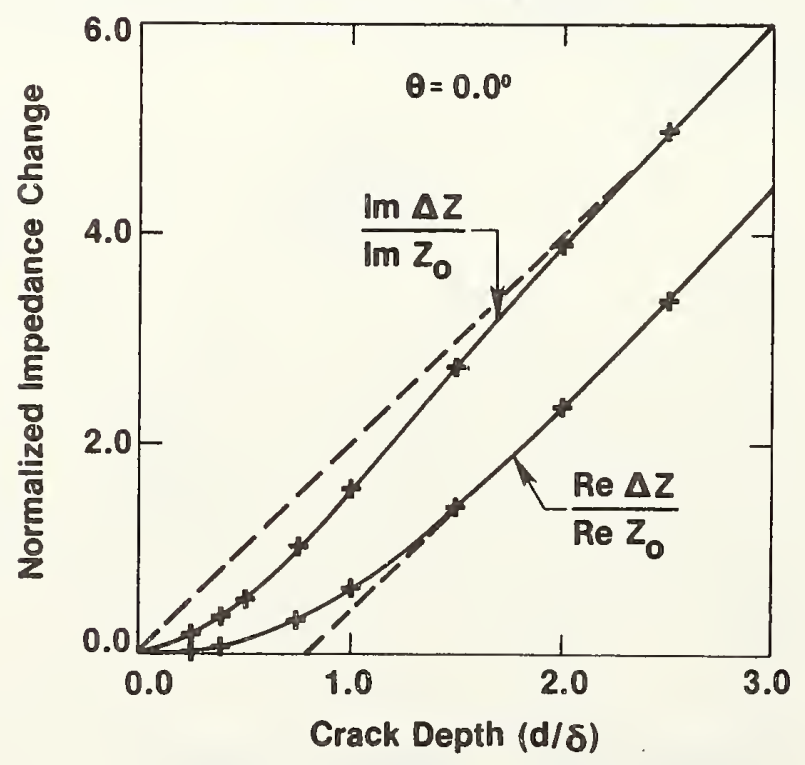

Fig. 25. Normalized total impedance change as a function of crack depth for a perpendicular crack. $(\delta=$ skin depth) 
Normalized Impedance Change

vs.

Crack Depth

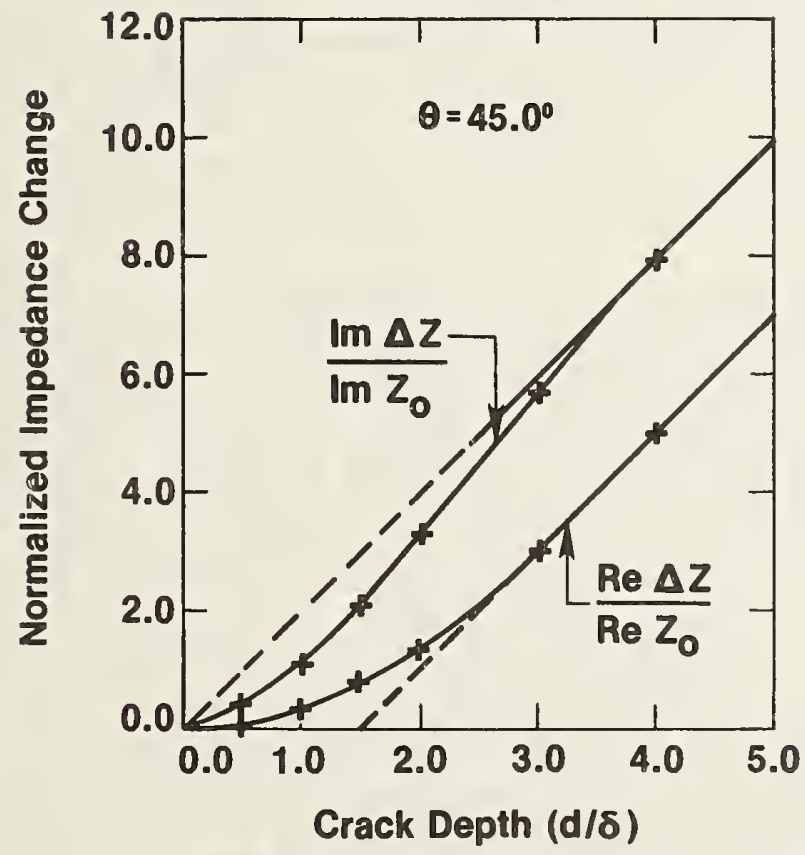

Fig. 26. Normalized total impedance change for a crack inclined at $45^{\circ}$ as a function of crack depth. ( $\delta=$ skin depth) 
The first phase of our effort toward improved reference standards was for the standard leak range of 1 to $1 \times 10^{-5} \mathrm{~atm} . \mathrm{cm}^{3} / \mathrm{s}$. The second phase was to extend the measurements range from $1 \times 10^{-5}$ to $1 \times 10^{-7} \mathrm{~atm}$. $\mathrm{cm}^{3} / \mathrm{s}$. We have obtained a capability to $1 \times 10^{-6} \mathrm{~atm} . \mathrm{cm} 3 / \mathrm{s}$ on an absolute basis. A concept under consideration for extending our measurement range to $1 \times 10^{-7} \mathrm{~atm} . \mathrm{cm}^{3} / \mathrm{s}$ is to employ a mixed gas passing through a larger leak, as measured by the rate-of-rise method, and the calibration of a mass spectrometer by the gas components. Under continuum flow, all gas components would be transported at the same rate so that the flow rate of any constituent would simply be that fraction of the total flow equal to its relative concentration. For example, a $1 \% \mathrm{He} / \mathrm{N}_{2}$ mixture in a $1 \times 10^{-5} \mathrm{~atm} . \mathrm{cm}^{3} / \mathrm{s}$ leak size capillary should produce a $1 \times 10^{-7} \mathrm{~atm} . \mathrm{cm}^{3} / \mathrm{s}$ partial leak rate of helium. However, standard leak rates are determined with one atm pressure upstream and zero pressure downstream, where no flow is completely continuum. Instead the nature of the transport mechanism changes along the length of the channel from laminar viscous flow at the entrance to combined slip flow and then free molecular flow as the exit is approached; thus, fractionation of the gas components can be expected. The effect of this fractionation on the accuracy of the expected partial flow rate is a prime issue for this method.

Theoretical guidelines for anticipated behavior are appropriate to reduce the amount of experimental effort that would be required otherwise. Unfortunately, the theory of gas flow through capillary tubes has only been treated satisfactorily in two distinct and separated pressure regions: the low pressure region of free molecular flow where the mean-free-path is large compared to the tube diameter; and the moderate pressure region of continuum flow where the mean-free-path is much less than the diameter but where the pressure is low enough to avoid turbulence. No solution has been obtained from first principles for the complete pressure range that is required for leak size measurement. In addition, both slip coefficient and the transmission probability of free molecular flow are significantly affected by wall condition, which factor has not been formulated theoretically. We have used two semiempirical formulations for analyses. One by Fryer(1), which modifies the viscous flow with slip formulation as based on a phenomenological argument, is appropriate for long tubes, does not account for wall condition, and is in differential form. It predicts the nature of the transport pressure dependence. The second, by Lund and Berman(2), is a superposition of diffuse and drift flows with modifying constants derived from a nonlinear least-square fitting to experimental data, is applicable to any length-to-radius value, and incorporates adjustable parameters to account for deviation from theoretical transmission probability and slip coefficient.

Detailed calculations have been made. The total flow rate, the fractional contribution to flow rate of the several flow mechanisms, the pressure profile along the capillary channel, and the pressure dependence of these 
single gas species. A systematic examination of practically obtained capillary dimensions indicates a severe constraint on choice. Figure 27 represents typical results for a series of capillary tube diameters and Table IV 1 ists the net values. The Fryer equation was first integrated to obtain the total flow rate for one atm $\mathrm{N}_{2}$ upstream and zero pressure downstream, and then the differential form was used in an iteration procedure to derive the profile along the tube lenath. Expected standard leak rate (leak size) is $3.35 \times 10^{-6} \mathrm{~Pa} \cdot \mathrm{m}^{3} / \mathrm{s}\left(\sim 3 \times 10^{-5} \mathrm{~atm} \cdot \mathrm{cm}^{3} / \mathrm{s}\right)$ of which $84.7 \%$ is laminar flow, $14.5 \%$ is slip flow, and $0.8 \%$ is molecular flow. Increasing the diameter increases the percentage of laminar flow, but then longer lenqths are necessary to reduce flow rate. For example, a $16 \mu \mathrm{m}$ diameter by $1-\mathrm{cm}$ length produces a $4.92 \times 10^{-5} \mathrm{~Pa} \cdot \mathrm{m}^{3} / \mathrm{s}$ flow rate with $92.1 \%$ laminar, $7.7 \%$ slip, and $0.2 \%$ molecular flows; however, we would need a capillary length of $\sim 10 \mathrm{~cm}$ to reduce the flow rate to the $1 \times 10^{-6}$ $\mathrm{Pa} \cdot \mathrm{m}^{3} / \mathrm{s}$ value desired, which length cannot be drawn with any feasibility For $16 \mu \mathrm{m}$ diameter, the laminar flow profile is extended closer to the exit as are the slip and molecular flows. Selection of long precision bore capillaries require reduced upstream conditions with no consequent improvement.

In order to assess the amount of fractionation with mixed gas flow, we have to resort to guesswork, for mixed gas flows under these conditions have apparently not been approached theoretically. To do this, we substituted values for mixed gas viscosities into the Lund and Berman formulation, selected midband values for slip coefficients, and chose appropriate values for collision parameters. The determination of mixed gas viscosities is usually not obtained from first principles because of the detailed collision analysis required, and suitable approximations or empirical formulas must be used. Experimental observations show that the net viscosity can be altered significantly by small concentrations of a second gas and not in readily predictable fashion for asymmetric molecules. We calculated the viscosities from a Chapman-Enskog(3) formulation and approximated collision cross sections with equivalent hard sphere diameters. Significant changes in flow are predicted. Only when the tracer gas is held to small concentrations are the changes reasonable. For example, a $1 \% \mathrm{He} / \mathrm{N}_{2} \mathrm{mix}$ would increase the flow rate in the $8-\mu \mathrm{m}$ diameter capillary by $\sim 5 \%$ but decreases the slip contribution by $\sim 1 \%$. Although the change in total flow rate could be measured by rate-of-rise, the amount of fractionation is not apparent from these formulations.

An alternate method which has been used to generate leak rates of less than $1 \times 10^{-5} \mathrm{~atm} . \mathrm{cm}^{3} / \mathrm{s}$ utilizes a porous column for which the pore diameter is much less than the mean-free-path of the upstream pressure. However, it may take repeated processing and periods of exposure of up to one year before the flow conductance stabilizes to $\sim 7$ percent. Replacement of the porous plug with a fixed length of precision bore capillary and precise low pressure generation upstream(4) to achieve pure molecular flow have been analyzed similarly as above. Again, selection of capillary dimensions to allow a measurable $10^{-6} \mathrm{~Pa} \cdot \mathrm{m}^{3} / \mathrm{s}\left(10^{-5} \mathrm{~atm} \cdot \mathrm{cm}^{3} / \mathrm{s}\right)$ ieak rate at the upper limit, while holding necessary pressures upstream, are constrained, but prognosis is more positive than for mixed gas flow. A third method utilizes a flow divider; this is susceptible to greater accuracy eventually but is more complex and requires extended apparatus. 


\section{References}

1. G. M. Fryer, "A Theory of Gas Flow Through Capillary Tubes," Proc. Roy. Soc., A293, 329-341 (1966).

2. L. M. Lund and A. S. Berman, "Flow and Self-Diffusion of Gases in Capillaries, Part I and Part II". J.A.P. 37, 2489-2495 and 2496-2508 (1966).

3. See E. H. Kennard, "Kinetic Theory of Gases", 1st ed., 4th impression (McGraw-Hi11, New York, 1938) p. 161.

4. S. Ruthberg, "Calibration to High Precision in the Medium Vacuum Range with Stable Environments and Micromanometer", J. Vac. Sci. Technical 6, 401-412 (1969).

Table IV

Leak size and net fractional flow for small diameter capillaries, 1-cm length, nitrogen gas

$\begin{array}{ccccc}\text { diam } & \text { leak rate } & \text { laminar } & \text { slip } & \text { molecular } \\ (\mu \mathrm{m}) & \left(\mathrm{Pa} \cdot \mathrm{m}^{3} / \mathrm{s}\right)^{*} & \% & \% & \% \\ & & & & \\ 4 & 2.26 \times 10^{-7} & 71 & 27 & 2 \\ 8 & 3.35 \times 10^{-6} & 84.7 & 14.5 & 0.8 \\ 16 & 4.92 \times 10^{-5} & 92.7 & 7.74 & 0.24\end{array}$

${ }^{*} \mathrm{~Pa} \cdot \mathrm{m} 3 / \mathrm{s}=9.872 \mathrm{~atm} \cdot \mathrm{cm}^{3} / \mathrm{s}$ 
Figure 27. Pressure and flow profiles for nitrogen gas through a series of capillary tubes of $4 \mu \mathrm{m}, 8 \mu \mathrm{m}$, and $16 \mu \mathrm{m}$ diameter by $1 \mathrm{~cm}$ length. One atm gas pressure is applied upstream and zero pressure is maintained downstream. The fractions in percent of laminar, slip, and molecular flow are indicated for any cross-sectional plane along the capillary length from the downstream exit.

molecular flow; -.-- slip flow; _- -- - laminar flow; - - - pressure.

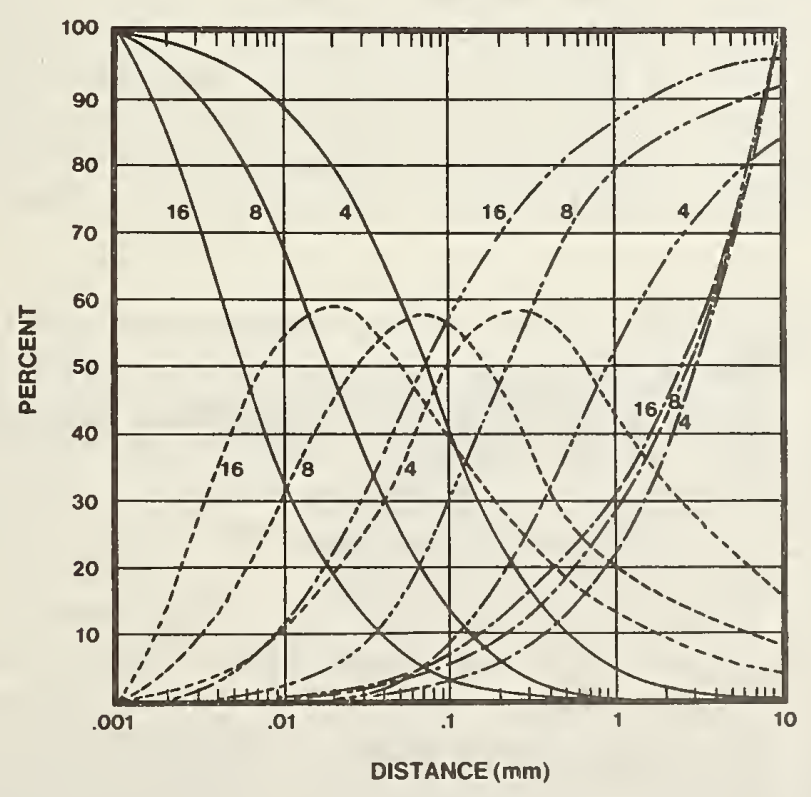

Fig. 27 
13. Magnetic Measurements

L. J. Swartzendruber

Metaliurgy Division

Center for Materials Science

Magnetic methods of nondestructive evaluation utilize the relationship between material properties and static or slowly varying magnetic fields. They find wide application both on the production line and in the field and, where useable, provide a sensitive, rapid, and often relatively inexpensive test method. Uses include measurement of important metallurgical properties, detection of harmful defects, and measurement of coating thickness.

One of the most extensively used techniques in magnetic NDE is the magnetic particle method. The application of this method is currently guided by empirical rules for the amount of magnetization required to achieve the desired results. Also, there is no acceptable method for independently testing magnetic inks. The first steps in placing magnetic particle inspection on a more quantitative and reproducible basis are in understanding of how the leakage field from a defect is influenced by the defect geometry and level of magnetization reached by the material, and a detailed description of the influence of the leakage field on the formation of an indication by the magnetic ink.

Previous investigations have led us to propose a detectibility criterion for defects under magnetic particle inspection. Using this criterion, and a phenomenological description of the magnetic leakage field from cylindrical defects, the detectibility of cylindrical defects as a function of defect depth, defect size, and applied magnetic field can be predicted. In order to apply this criterion, and to test its validity for more complicated geometries, it is necessary to calculate leakage fields from more complicated geometries. Computer modeling techniques to perform this function are being developed.

Results have been obtained from a finite difference model using a square grid and defects with a square cross section placed at various depths below the sample surface. In the initial form of this model a linear isotropic magnetic medium was assumed. After the model results were obtained they were least-squares fitted to the field expected from a magnetic dipole. (The field from a cylindrical defect in a linear isotropic medium can be calculated analytically and is a dipole field.) Figures 28 and 29 show the fields perpendicular and parallel to the surface of the material, respectively. It can be seen in these figures that the field from a square defect is aimost indistinguishable from a dipole field. Note that the field parallel to the surface has a large bias field of 30 gauss. This added field is just the applied field which was parallel to the surface. Using these results a quantitative comparison can be made with the field from a cylindrical defect. The square defect gives a leakage field approximately ten percent higher than that from an inscribed cylinder. 
It is planned to continue these calculations for different geometries such as rectangles and slots. In addition, the program is being modified to cover the more realistic case on nonlinear media. For this case, a finite element method will be employed in an attempt to reduce the expected large increase in computer time. The outcome of these calculations, along with further experimental measurements, will be used to develop a critically needed specification for the applied field necessary to detect defects of a given size and geometry. The results will also be useful for a leakage field measurement method which is a technique of growing use in the field of nondestructive evaluation.

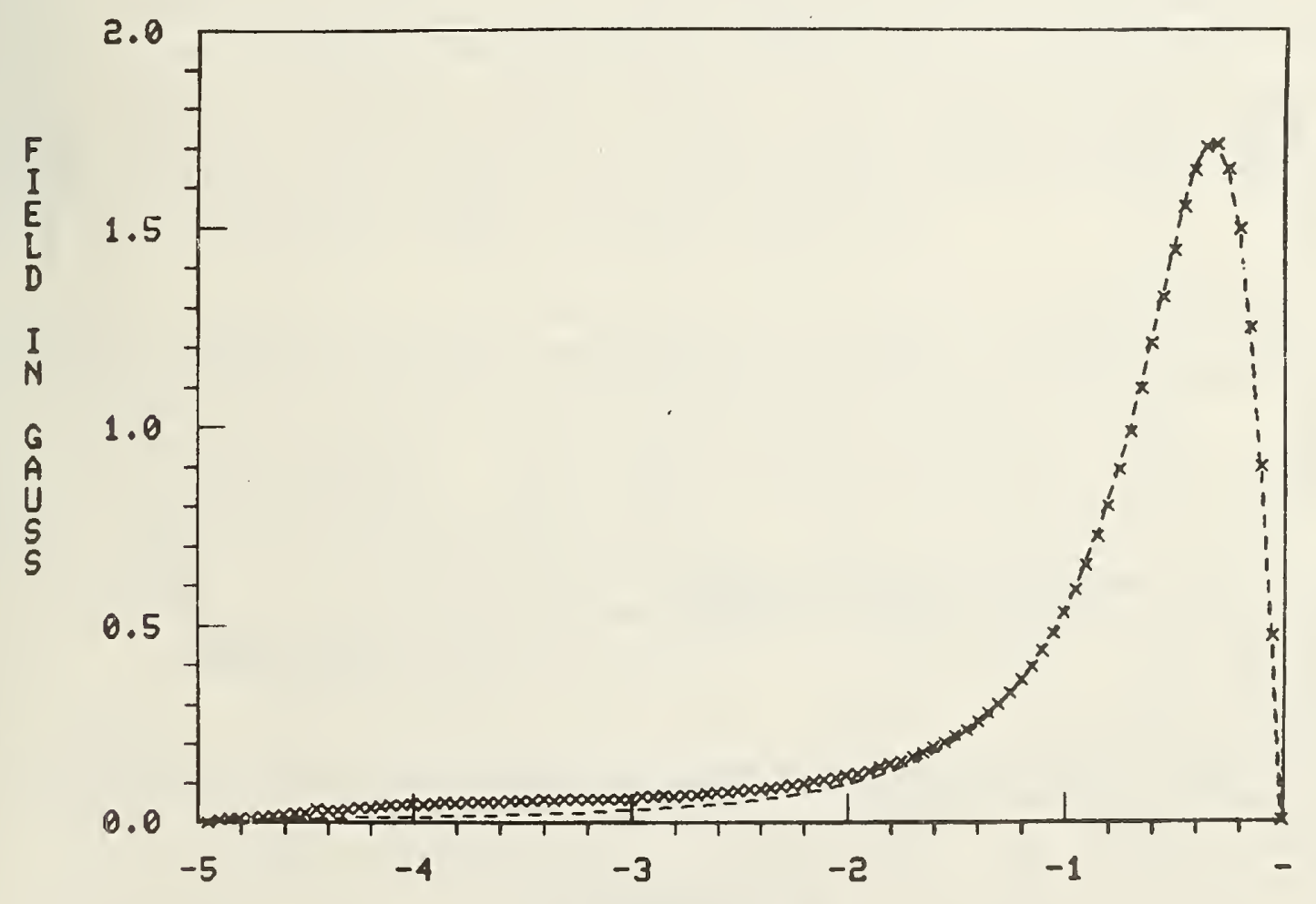

DISTANCE IN CM.

Fig. 28. Magnetic leakage field perpendicular to the sample surface versus distance from the defect center (the field is antisymmetric about the origin; only the left half is shown). The applied field is 30 gauss parallel to the sample surface and the leakage field is assumed to be measured $0.3 \mathrm{~cm}$ above the surface. The defect cross section is a square $0.2 \mathrm{~cm}$ below the surface. The crosses are the calculated field and the dashed line is a least squares fit to a dipole field. 


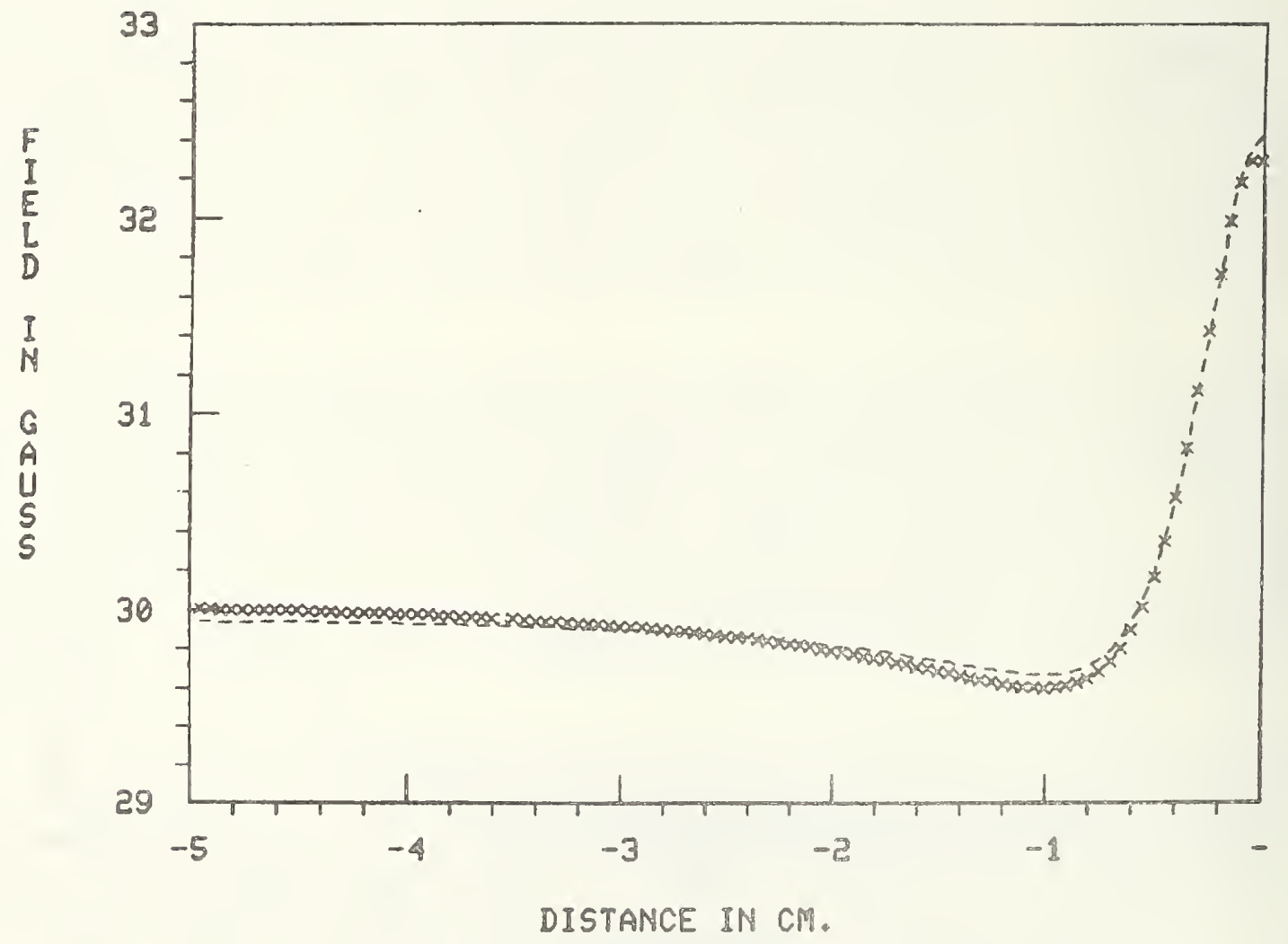

Fig. 29. Magnetic leakage field parallel to the sample surface versus distance from the defect center (the field is symmetric about the origin; only the left half is shown). The applied field is 30 gauss parallel to the sample surface and the leakage field is assumed to be measured $0.3 \mathrm{~cm}$ above the surface. The defect cross section is a square $0.2 \mathrm{~cm}$ on a side with the top $0.2 \mathrm{~cm}$ below the surface. The crosses are the calculated field and the dashed line is a least squares fit to a dipole field. 
14. Optical Measurement of Surface Roughness

E. Clayton Teague, Theodore Vorburger, Fredric Scire, Saul Baker, and Clare Trahan

Mechanical Production Metrology Division

Center for Manufacturing Engineering

There have been, over the last ten years, increasing efforts in the U.S. and the rest of the world to use light scattering rather than the traditional stylus instrument for nondestructive evaluation of the surface roughness of manufactured parts. The roughness of these surfaces typically has irregularity spacings of 10 to 1000 times that of visible light wavelengths, $\lambda$, and irregularity heights of $0.25 \lambda$ to $10 \lambda$. The theory of light scattering from surfaces with such properties is not well understood. The quantitative measurement of surface roughness characteristics from light scattering measurements in this regime is, therefore, not feasible with available information. To meet this need for improved information about light scattering from manufactured surfaces and to facilitate the anticipated transition from stylus to 7ight-scattering measurements we have begun both theoretical and experimental investigations of light-scattering techniques.

Toward the objective of establishing a solid basis for developing recommended methods for optical measurement of surface roughness we have: (1) Completed and published (1) the results of an extensive 1 iterature search and analysis of the papers on experimental optical techniques for measuring surface roughness. This paper (1) reviews state-of-the-art optical techniques including specular reflectance, total integrated scatter, diffuseness, angular scattering distributions, speckle, ellipsometry, and interferometry. (2) Completed an evaluation and distillation of available theoretical models that can be used to describe light scattering from rough surfaces. Results of this work (2) appear in the September 1981 issue of Annals of CIRP. (3) Designed a system for performing angular distribution measurements of light scattered from a test surface. Al1 major engineering drawings and, with the exception of a fiber optic-detector array, al1 major components of the system have been procured and are now being integrated into a total system; completion and first operation of the system is planned for December 1981. It is described more fully below. And (4) designed and constructed a high-precision $x-y$ stage for use in obtaining 3-D stylus profilometry images for the microtopography of a specimen surface. Finally, a general survey of optical nondestructive evaluation techniques was written and will be published next year (3).

A schematic of the system being constructed for performing angular distribution measurements is shown in Fig. 30. The system has been designed to serve as a versatile research instrument which permits measurements of the intensity of scattered light as a function of azimuth and elevation over the full hemisphere above the test surface. In addition, the system was designed to serve as a prototype on-line measuring device which allows a rapid snapshot of the angular distribution of light scattered in the plane of incidence. As shown in Fig. 30, the system consists of an array of 90 detectors interfaced through a 100-channel scanner/digital voltmeter to a Hewlett-Packard 9845T desktop computer. To facilitate the comparison of light scattering and stylus measurements of surface roughness, the HP9845T computer is interfaced with an RS-232 communication link to the computer, an 
Interdata 7/32, which is being used to acquire, display and analyze the 3-D stylus profilometry measurements.

The goal of the combined stylus/light scattering measurements is to measure a designated area of a test surface with iight scattering, compute from these measurements statistical characterizations of the surface roughness, such as the 2-D power spectrum, relocate the identical area for 3-D stylus profilometry and compute the same statistical characterizations from the stylus measurements. Comparison of characterizations from the two methods should then enable us to build a solid empirical base for developing better theoretical models for light scattering from manufactured surfaces.

The optical and mechanical aspects of the detector array for lightscattering measurements are shown more completely in Fig. 31. Fiber optics are placed on the yoke at angular intervals of two degrees with each aperture subtending an angle of 1.2 degrees. For measurements in the plane of incidence, defined by vectors along the incident light path and the surface normal, the angles of incidence will also be confined to multiples of two degrees because light must travel to the specimen along paths between the fiber-optic connectors. When the yoke of the fiber optics is rotated to permit measurements out of the plane of incidence, higher resolution measurements can be performed if necessary. If measurements in the plane of incidence at resolutions greater than two degrees are required for specific tests, the laser light source can be manually repositioned to permit measurements at intervals of less than 0.1 degree. The collected light as a function of angle is then transmitted by the fiber-optic cables to an array of photodiodes and amplifiers which, in turn, provide voltages that are input to the 100-channel scanner. By allowing the placement of the detectors in a compact isolated electrical unit, the fiber-optic approach minimizes any potential problems with electrical noise which could be very troublesome at the low levels expected for these measurements.

The $x-y$ stage for obtaining 3-D stylus profilometry maps of surface microtopography moves the specimen in a rastered motion beneath a fixed stylus location. As the specimen is moved beneath the stylus, the stylus "vertical motion is used to produce an electrical signal. This signal is converted to a digital value at a $512 \times 512$ array of $x-y$ positions. From this array of digital values an image of the topography is generated in which the intensity at each point is proportional to the surface height of the specimen at the corresponding point. Three-D stylus profilometry images have proven to be very useful in revealing features of the topography not easily detected with other methods, including electron microscopy. The quantitative representation of topography in these images is unique. Our present efforts in this area are to generalize the algorithms we have developed for characterizing 2-D stylus profiles to enable the computation of new characterizations applicable to 3-D topography. We are also carefully measuring the geometric errors present in the motion of the $x-y$ stage, i.e., pitch, roll yaw, and vibration. 


\section{References}

1. T. V. Vorburger and E. C. Teague, "Optical Techniques for On-Tine Measurement of Surface Topography," Precision Engineering 1981, $3,61-83$.

2. E. C. Teague, T. V. Vorburger, and D. Maystre, "Light Scattering from Manufactured Surfaces," Annals of CIRP, September 1981.

3. E. C. Teague, T. V. Vorburger, and G. Birnbaum, "Optical Nondestructive Evaluation," to be published in Encyclopedia of Materials Science and Engineering in 1982. 


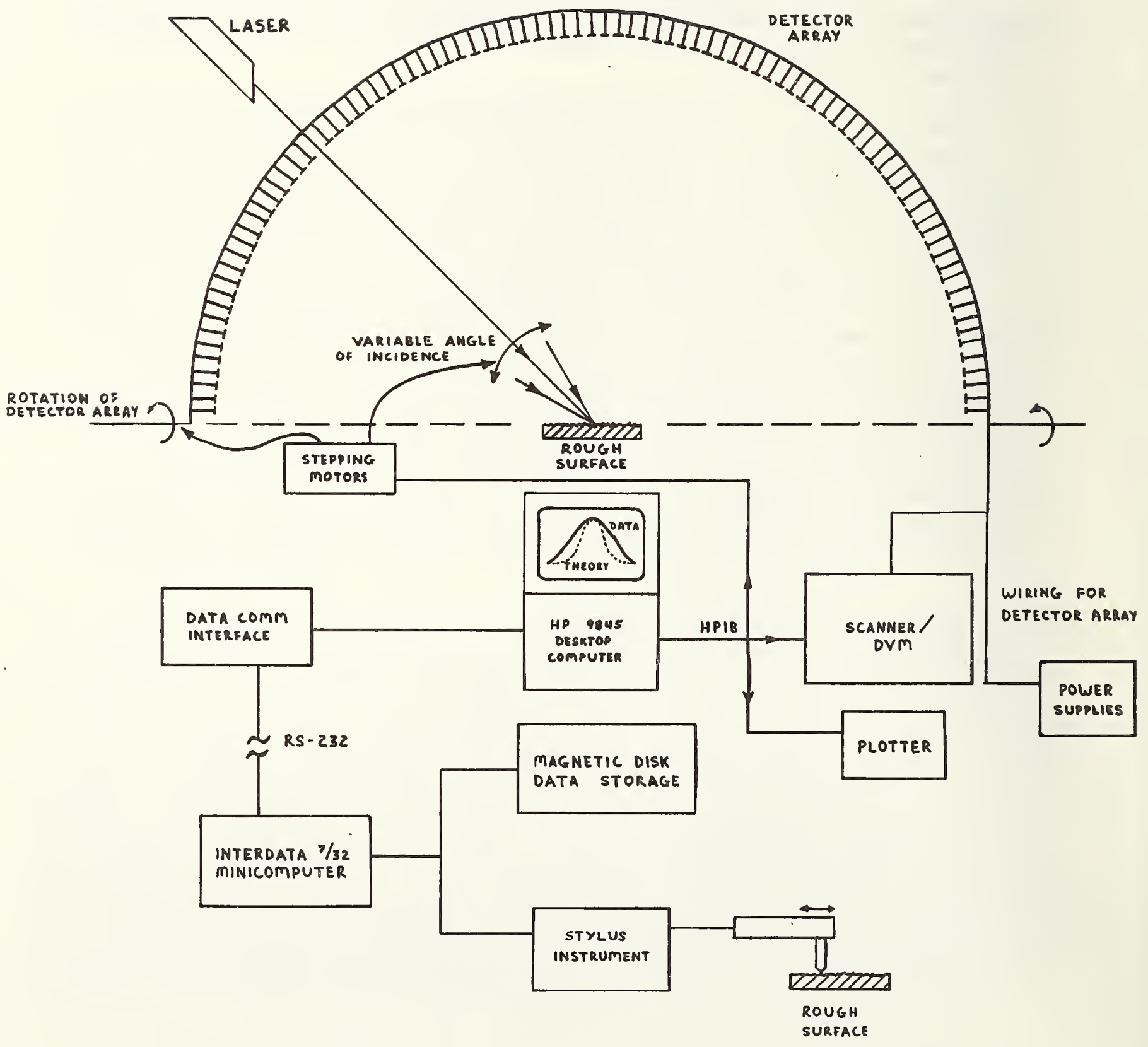

Fig. 30 Angular scattering systel: for measuring surface roughness 


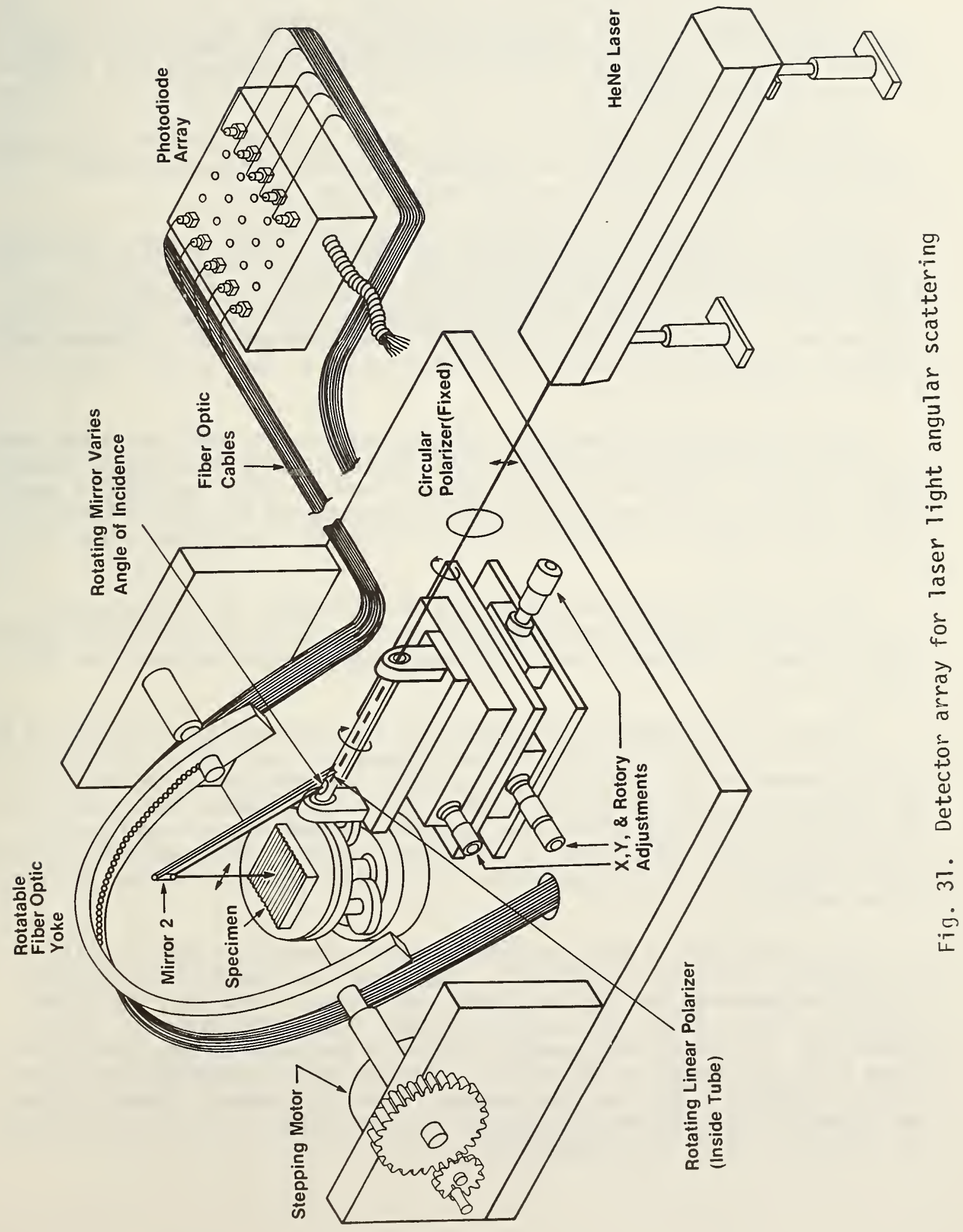


15. Optical Scattering for Nondestructive Evaluation

A. Feldman and G. S. White

Ceramics, Glass and Solid State Science Division

Center for Materials Science

Using the apparatus developed last year for measuring the scatter as a function of angle for $10.6 \mu \mathrm{m}$ wavelength radiation incident upon surface grooves, data were taken of scattering from shallow rectangular and deeper $\mathrm{V}$ - and trapezoidal grooves.

The scalar model developed for the scattering from shallow (depth $\sim 0.05 \lambda$ ) grooves accurateiy described the observed scattering and quantitatively incorporated certain possible misalignments of the system.

Three deep $V$-shaped and three deep trapezoidal shaped grooves also were studied (Fig. 32) and scattering data were collected with the incident electric field both parallel and perpendicular to the groove length. The groove itself was normal to the scattering plane swept by the detector. Differences in the scattering with polarization were observed. However, the scattered fields maintained the same polarization as the incident field with no measurable cross-over.

Scalar calculations used to fit scattering from the shallow grooves were completely inadequate for describing the scattering from these deeper grooves. Daniel Maystre, a visiting French scientist from the CNRS, developed a vector theory which could be used to calculate the scattering from any cylindrical cross section due to an incident electric field with $E$ polarized parallel to the cylinder axis.

With the help of Jay Marchiando and Ted Vorburger, in the Mechanical Production Metrology Division, the cross sections of the grooves were measured with a diamond profilometer and preliminary calculations were made for the expected scatter.

Figure 33 is a composite of scattering data measured as a function of angle for several locations along the narrow V-groove (crosses) and the calculated scatter intensity (solid line). The calculations were made by measuring the groove cross section at one position with a profilometer and calculating the scatter from that profile. The agreement between the observed and the calculated scatter intensities is excellent. Experimentally observed asymetries are reproduced in the calculations at the correct angular positions and with proper magnitudes.

The variability in the experimental data reflects the lack of uniformity of the groove. This lack of uniformity also causes the asymmetries observed in both the measured and the calculated scattering. The brass, during the scribing process, flows into mounds on either side of the grooves. These mounds are, in general, not symmetric and also change in height, width, and shape along the length of the grooves. Another feature contributing to the lack of symmetry in the observed scatter is that the grooves themselves are not symmetric but have one side steeper than the other. All of these effects are accounted for in the calculations. 
Similar calculations have been made for the medium-sized $V$-groove and the narrow and medium-width trapezoids with a $20-\mu \mathrm{m}$ bottom. In these cases, ideally shaped grooves were incorporated into the calculations because the depths of these grooves were greater than the range of the profilometer. Nevertheless, the calculated scattering presented the same qualitative features, including number and approximate sizes and peaks, as those which were observed experimentally. Quantitative features, such as angular positions of the maxima and minima and observed asymmetries, are not yet in agreement with experiment. However, it has been found that these features vary significantly with relatively minor $(\sim 0.1 \lambda)$ changes in groove shape.

Currently, work is progressing in fitting these data more quantitatively. In the same vein, scattering from known positions on the narrow $V$-groove samples is being compared with that predicted from profilometer measurements at the same positions. Some work is also being done on the inverse problem, using calculated phases and experimentally determined amplitudes in an attempt to reconstruct, mathematical1y, surface defects. 


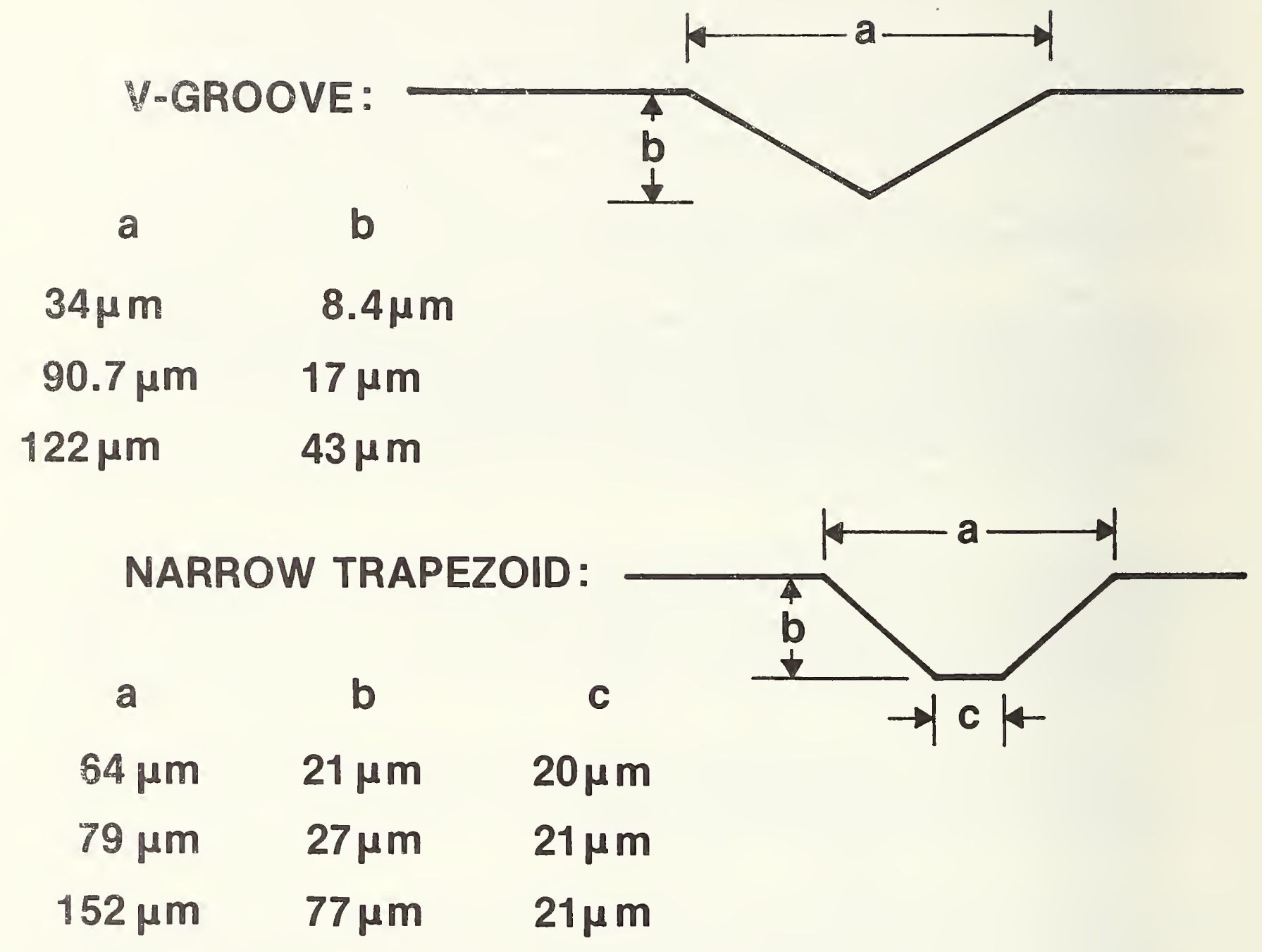

WIDE TRAPEZOID:

a

b

C

$89 \mu \mathrm{m}$

$15 \mu \mathrm{m}$

$51 \mu \mathrm{m}$

$128 \mu \mathrm{m}$

$57 \mu \mathrm{m}$

$51 \mu \mathrm{m}$

$48 \mu \mathrm{m}$

$69 \mu \mathrm{m}$

$51 \mu \mathrm{m}$

Fig. 32. Cross sections and nominal dimensions of ideal deep V-grooves
and trapezoids. 


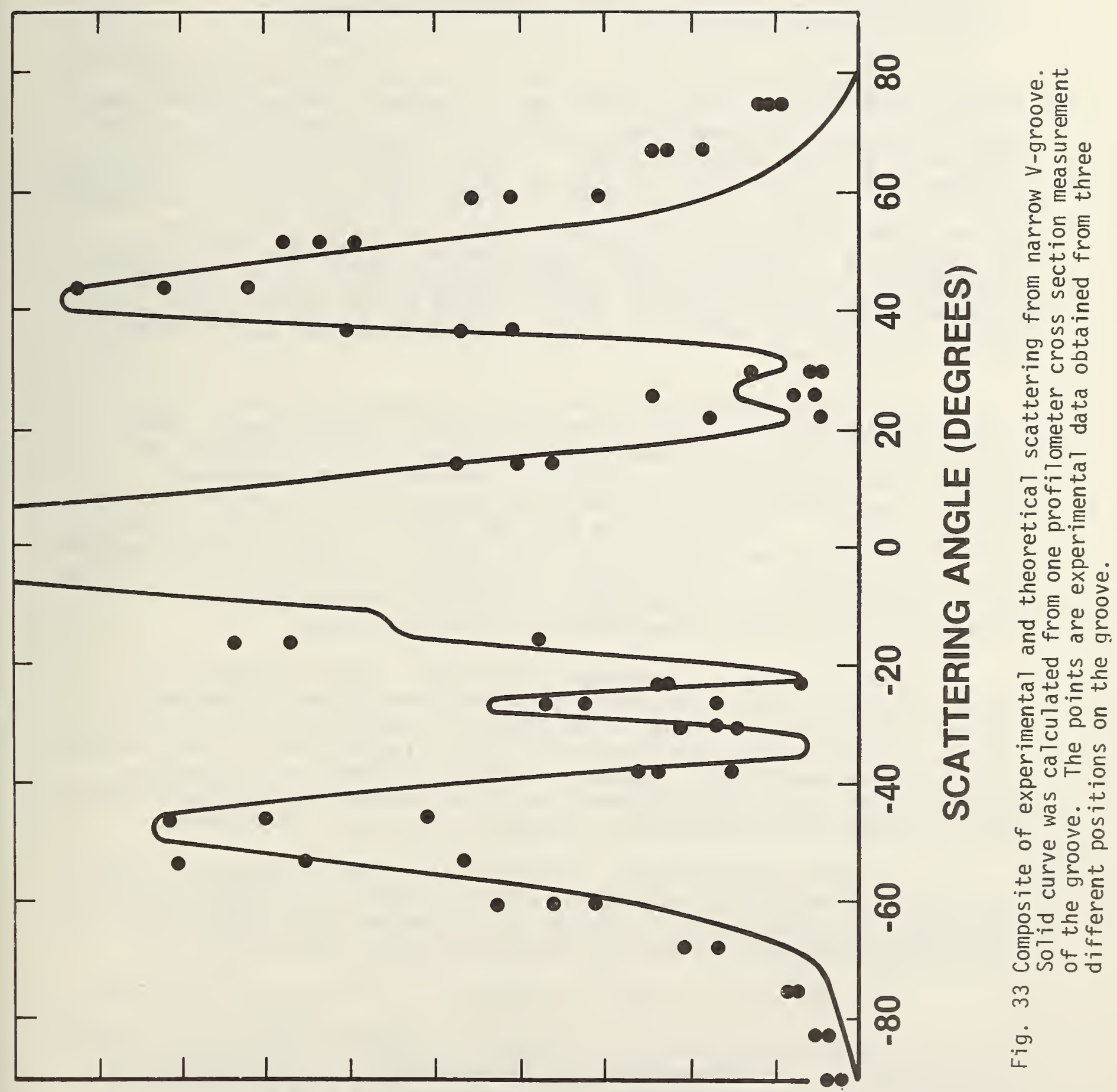

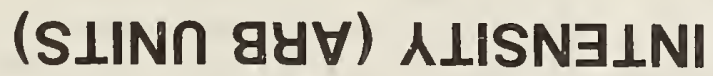


16. Signatures for Holograph NDE*

C. M. Vest

Dept. of Mechanical Engineering \& Applied Mechanics

The University of Michigan

The primary objective of this research program is to experimentally produce a series of "flaw signatures" of test objects with programmed flaws by holographic interferometry and to compare them with predictions made by the use of finite element analysis. The object under study has been an aluminum disk, outer radius $R=3.25 \mathrm{in}$. and thickness $t=0.25 \mathrm{in}$. The "flaws" are flat bottom holes of various radii, $r$, and depths, $d$, drilled from behind at the center of the disk. This radially-symmetric configuration was chosen because the costs of computing deformation are greatly reduced by the symmetry. In addition, the radially symmetric "flaw" is the "worst case" experimentally because the flaw is placed at the region of the lowest deformation gradient and because the flaw is concentric with the basic fringe pattern.

A test rig was assembled in which dry nitrogen gas can be used to pressurize the aluminum disks uniformly over their back surfaces. Each disk can be bolted by eight bolts, equally spaced on a 3 -in. radius, and sealed by an 0 ring against the housing. Four disks were fabricated; two had flaw diameters of $1-i n$; one had a depth of $1 / 8$ in. and one had a depth of $3 / 16 \mathrm{in}$. Two other disks had flaw diameters of $0.5 \mathrm{in.}$; one had a depth of $1 / 8 \mathrm{in}$. and one had a depth of $3 / 16 \mathrm{in}$. A steel ring was machined for use as a clamping surface around the periphery of the test plates. Using this clamping ring and a torque wrench to ensure uniform loading by the eight bolts, we obtained interferograms of the test plates.

In order to compare experimental and finite element results some normalization is required because the finite element solutions assumed a $2.5 \mathrm{in}$. radius, corresponding to the centers of the bolts, while a $2.56 \mathrm{in}$. radius, corresponding to the inner edge of the clamping ring, is more appropriate for the experimental results. It should be noted that the maximum deflection of a plate with clamped edges is proportional to the fourth power of the radius, so the results are strongly influenced by this choice.

i) Finite Element Results: Figure 34 is a plot of $W / W_{0 F E}$ versus $R / R_{0}$ where $W_{O F}$ is the maximum deformation of a plate with no flaw ${ }^{2}$ predicted by the $P E_{n}$ ite element analysis, and $R$ is the radius of the clamped edge. The dimensions and material properties used to generate these results are:

$$
E=10^{7} \text { psi, } \mu=0.333, t=0.25 \text { in., } R_{0}=2.5 \mathrm{in}, P=58.61 \text { psi }
$$

ii) Experimental Results: Figure 35 is a plot of $w / w_{0}$ versus $R / R_{0}$ for four test disks. The measured normal displacement, $w$, is normalized with respect to $w_{0 T}$, the displacement of the center of the disk predicted by plate theory. The displacement, $w$, is deduced from holograms.

The outer radius of the plate, $R$, was taken to be the inner radius of the steel clamping ring. When Figures 34 and 35 are compared, significant differences are noted between the finite element predictions and

*Work performed under contract to the NBS Office of Nondestructive Evaluation. 
the experimental results, particularly for the disk with the largest flaw. Although the accuracy of the finite element solutions may be questioned to some extent, the most likely cause of the discrepancy is incomplete realization of the "clamped" boundary conditions and subsequent improper normalization of the displacement. (Recall that $w$ is proportional to $\mathrm{R}^{4}{ }^{4}$.) This interpretation is supported by Fig. 36 in which $w / w_{0 E X P}$, where $w$ is the measured displacement and $w_{0 E X P}$ is the measured maximum displacement for the test plate with the largest flaw, is compared with the corresponding values determined by finite element analysis. These curves are in quite close agreement. The results for flaw free plates are also shown in this figure.

This work was undertaken to determine if holographic NDE results can be quantitative. The quantitative agreement between theory and experiment which was obtained is encouraging, although the sensitivity of the results to the exact loading configuration emerged. Future work will be concerned with other flaw geometries and locations. 


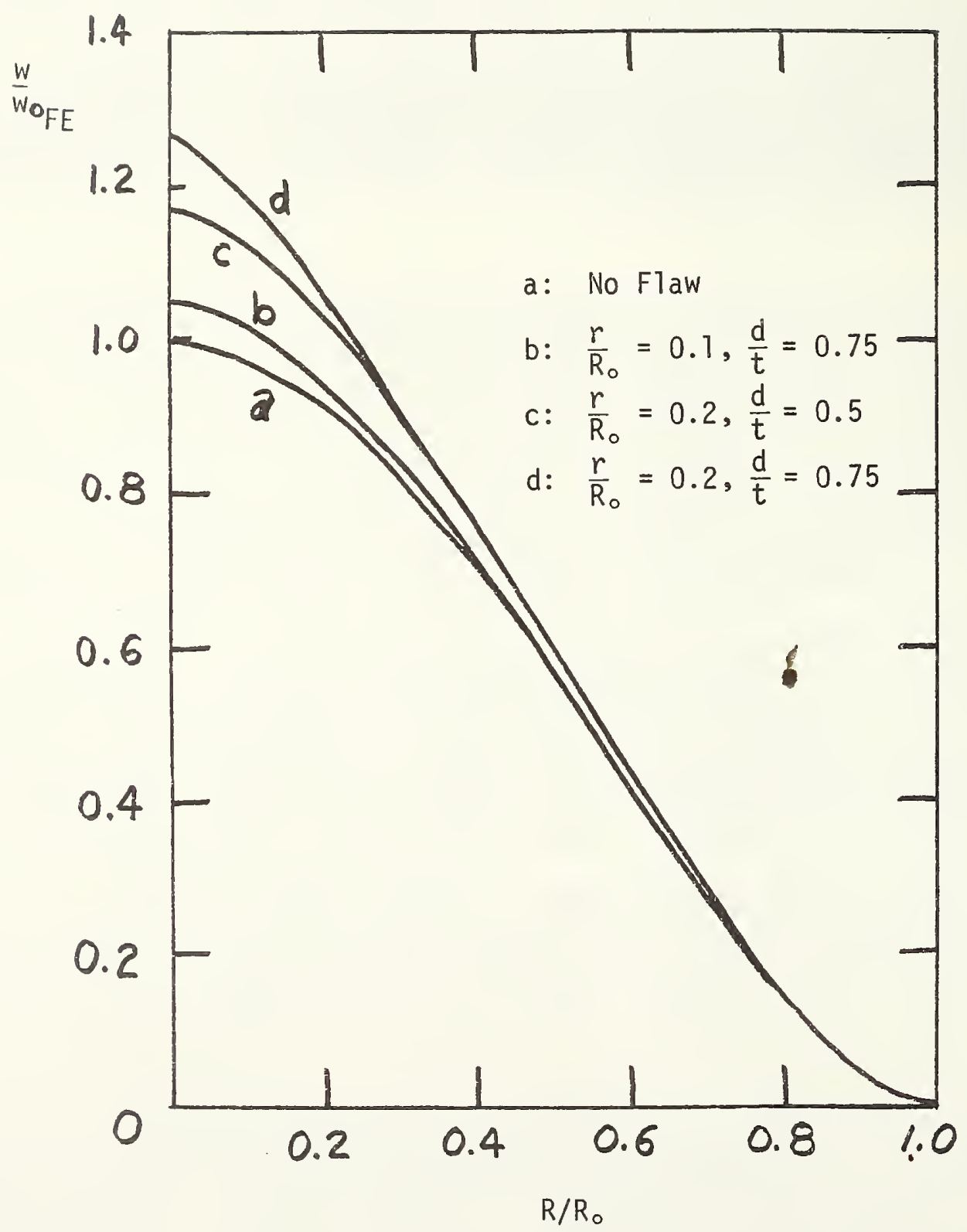

Fig. 34. Effect of flaws on normal deformation w (finite element computation). 


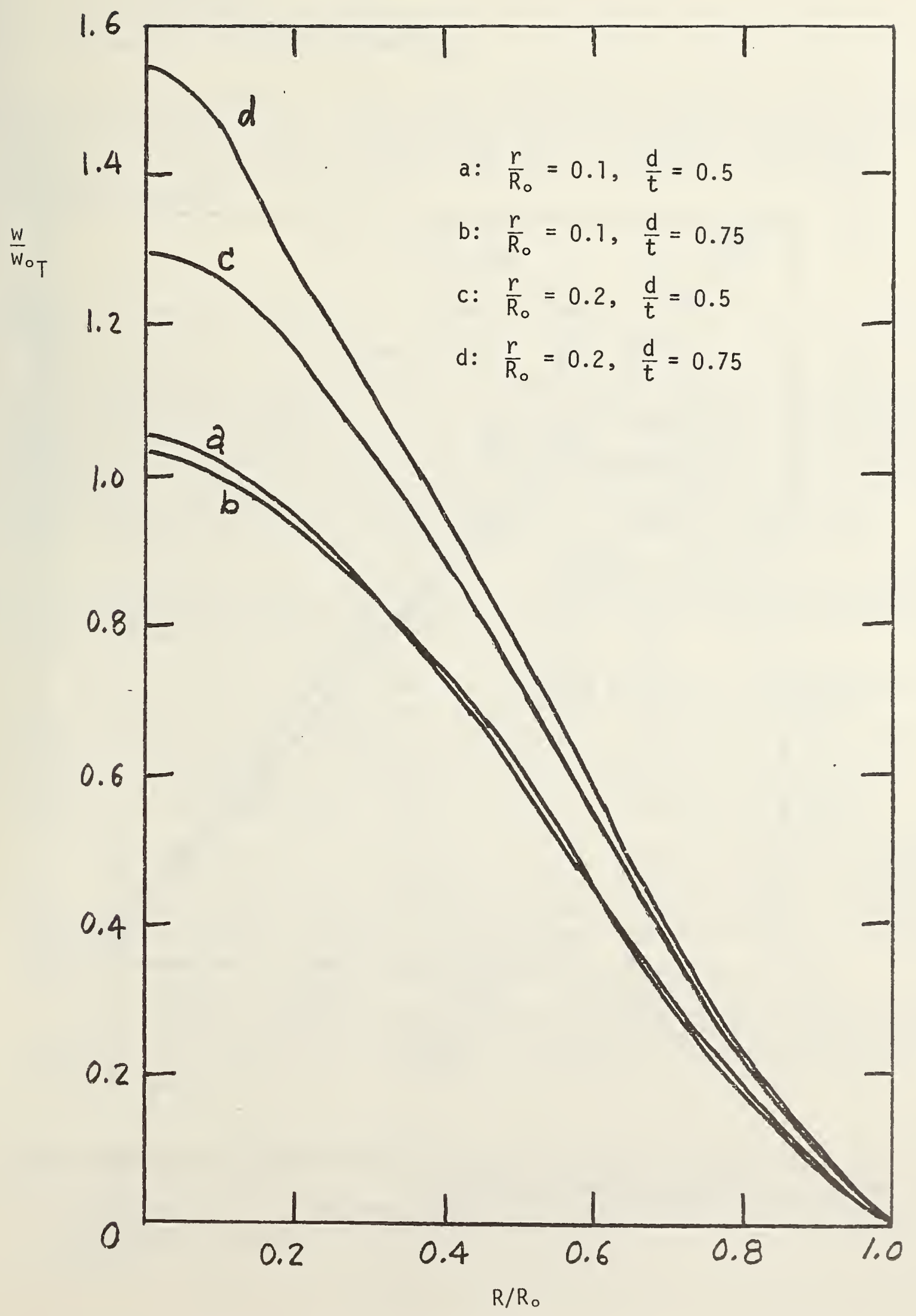

Fig. 35. Effect of flaws on normal deformation w (holographic interferometry). 


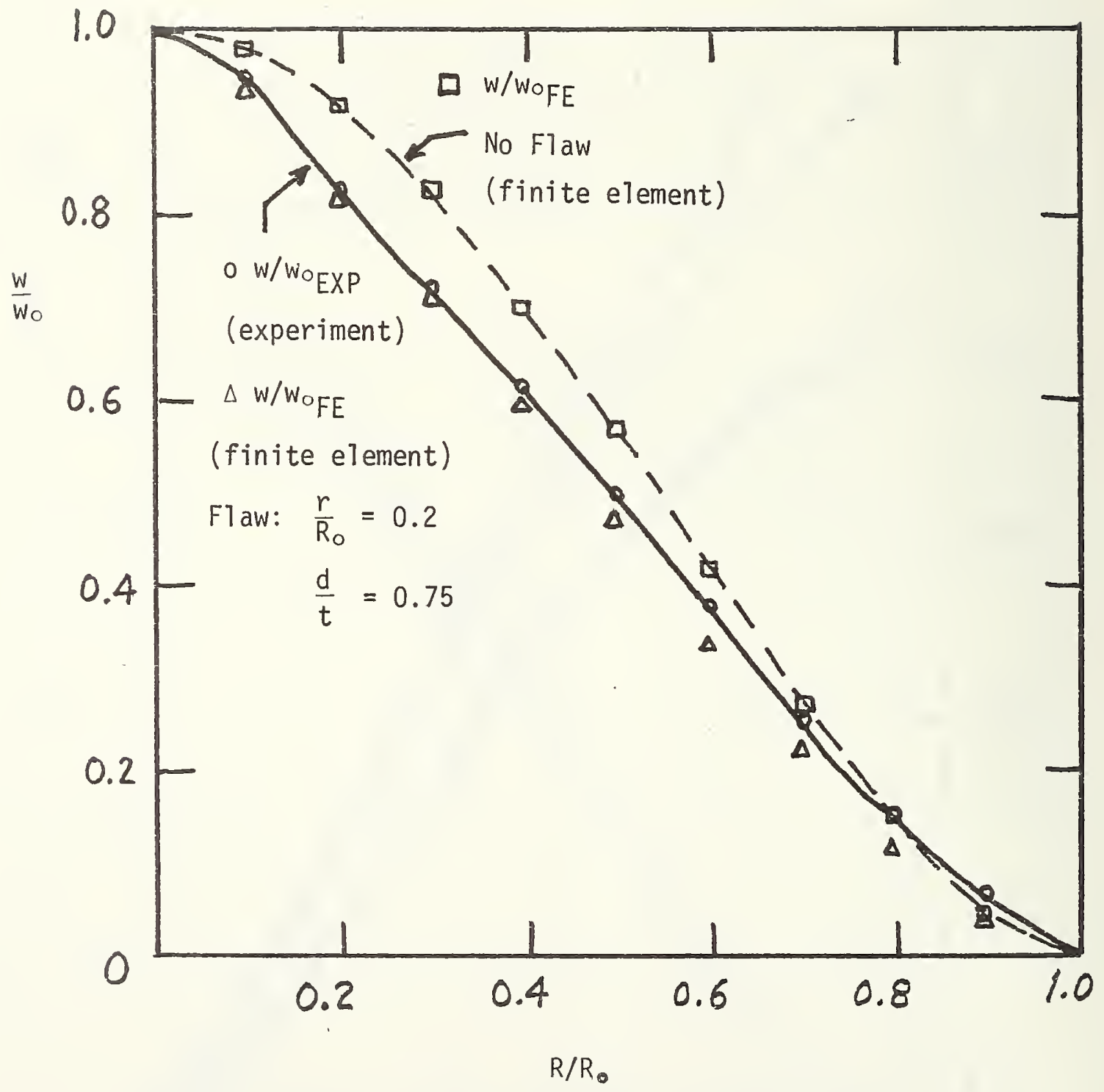

Fig. 36. Effect of flaws on normal deformation w (comparison of holographic interferometry and finite element results) 
17. Fluid Dynamics of Liquid Penetrant Flows

J. M. McMichael

Fluid Engineering Division

Center for Chemical Engineering

Liquid penetrants are often used to detect small surface cracks in various solid materials. A preliminary study* showed that once the penetrant reaches a depth ( $\ell$ ) which is Targe compared to a characteristic measure of the crack width $(R)$, penetration time $(t)$ is governed by the Ridea TWashburn relation

$$
t \sim \frac{e^{2} \mu}{\alpha R \cos \theta},
$$

where $\alpha$ is surface tension, $\mu$ is viscosity, and $\theta$ is the contact (wetting) angle.

However, this result implies an infinite initial penetration velocity, which is obviously incorrect. This singular behavior results from neglect of fluid inertial effects during the initial flow development for smali times, and it may lead to significant underestimation of penetration time. In addition, convective accelerations in the entry region of the crack will, in principle, decrease the effective pressure gradient which drives the flow, resulting in further underestimation of penetration time.

Initial studies were also limited to idealized geometries with uniform crack dimensions. However, variations in crack width along the direction of flow may also affect estimates of penetration time.

Current work is directed toward evaluating these two fluid dynamical aspects of liquid penetrant flows. The problem is posed as an initial value problem for time-dependent two-dimensional flow, and considers the effect of the reservoir of penetrant initially applied to the surface of the material. Asymptotic techniques are being applied to examine both the initial flow development, and the effects of variable crack width.

A complete solution of the general problem requires numerical simulation of the flow field with special attention to the interface boundary conditions which involve a moving contact line with dynamic contact angles. Estimates based on the asymptotic analyses underway should indicate the extent to which such detailed numerical analysis is warranted.

An additional question currently being studied is whether the flow remains two dimensional, or whether the advancing interface is susceptible to an instability resulting in the escape of trapped gases along some portion of the crack length.

S. Deutsch, "A Preliminary Study of the Fluid Mechanics of Liquid Penetrant Testing," Journal of Research, Vol. 84, No. 4, July-August 1979. 
18. Use of Uranium-Doped Glasses for the Calibration of Apparatus Employed

in the Evaluation of Fluorescent Dye Penetrants

R. W. Burke and D. K. Hancock

Inorganic Analytical Research Division

Center for Analytical Chemistry

Fluorescent dye penetrant testing is used extensively for the nondestructive detection of flaws in a wide variety of test objects. As currently practiced, the procedures lack both reproducibility and reliability. Undoubtedly, at the present state-of-the-art, many components with damaging flaws are now passed while others are wrongfully rejected.

In order to provide a more valid basis for using fluorescent dye penetrant testing as a reliable screening device, work has been undertaken to evaluate the use of uranium-doped glasses as comparative fluorescent standards. Two forms of standards are currentiy in different stages of preparation and evaluation. The first type consists of a single, rectangular piece of Corning 3750 glass mounted in a metal heat sink. An engineering drawing of this assembly is shown in Fig. 37. The main body of the metal block is 2024 aluminum that has been anodized flat black and the bottom plate is brass. The slot milled in one half of the aluminum holder has been precision machined to provide a close fit with the glass. Final positioning of the fluorescent glass exactly flush with the top of the holder is accomplished under a microscope. It is subsequently held in place by filling the $1 \mathrm{~mm}$ void between the bottom of the glass and the brass plate with a black adhesive. Ten of these units have been built thus far and are now undergoing extensive homogeneity and accelerated stability testing. Initial measurements obtained by mapping the surface of these glasses in grid-fashion with a microspectrofluorimeter having a circular measuring aperture of $25 \mu \mathrm{m}$ indicate that the fluorescence does not vary more than two percent.

In addition to the ten heat sink units, two other units modified for water thermostating have also been fabricated. These will be used to measure accurately the temperature coefficient of the uranyl fluorescence in this particular batch of glass. As soon as the above studies are successfully completed, these assemblies will be sent out to the manufacturers and users of fluorescent dye penetrants for further evaluation and comment.

A second type of standard in the early stages of design and production employs glass fibers having varying uranium concentrations and diameters in order to obtain a graded series of fluorescent intensities and widths of indication. Fibers having nominal uranium concentrations of 0.6 to 2.5 weight percent and diameters of 5 to $50 \mu \mathrm{m}$ have been obtained.

Present plans call for making two variations of these fiber standards. One will have a fixed uranium concentration and varying diameters that approximate the widths of the indications commonly obtained in crack evaluation; the other will consist of a series of fibers having a constant diameter and a varying uranium concentration. Each type will be sandwiched between two nonfluorescent quartz plates with a transparent cement whose refractive index closely approximates the refractive index of the fiber. The diameter and relative fluorescent intensity of each fiber in a series will be specified. The final production, characterization, and testing of these fiber standards wi11 form the main thrust of our effort in FY 82. 


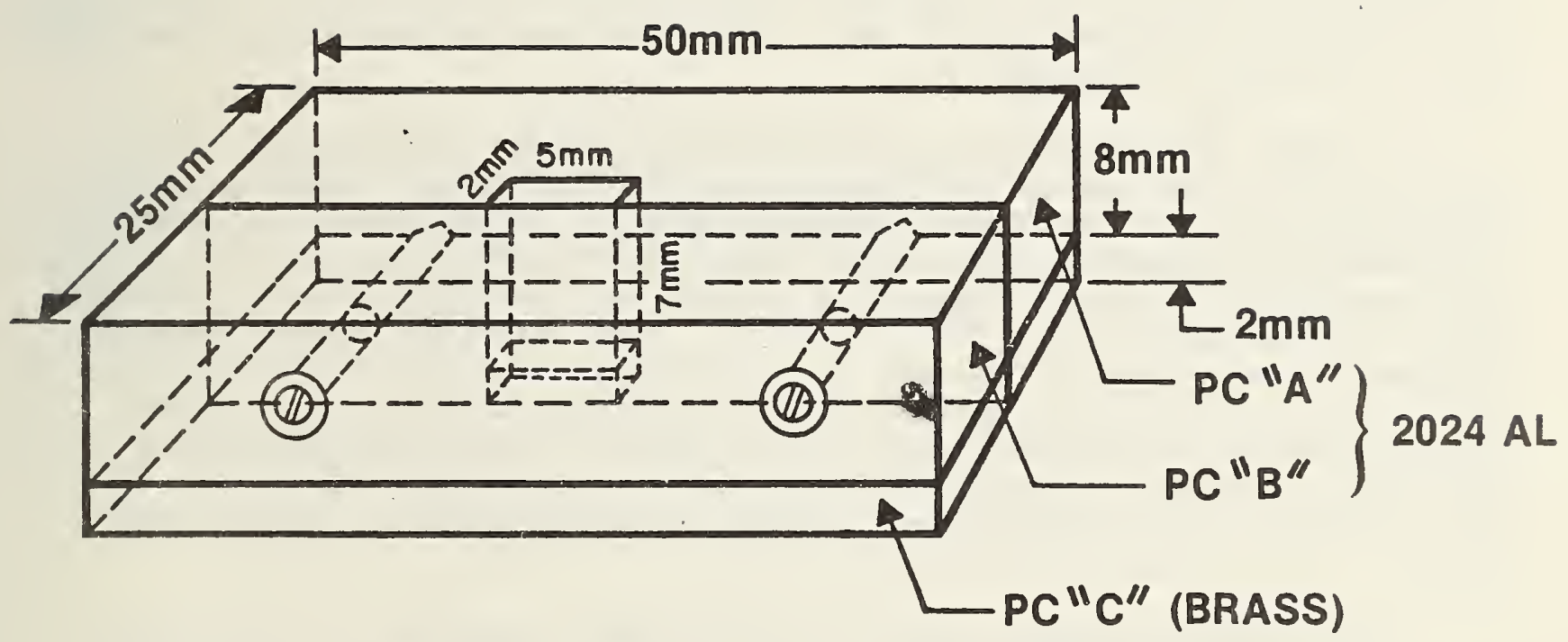

Figure 37. Fluorescent uranium glass and heat sink assembly. 
19. Mechanical Properties Characterization for Quality Control and NDE of Polymers

Donald L. Hunston and James L. Rushford

Polymer Science and Standards Division

Center for Materials Science

Satisfactory performance of a polymeric material in a practical application is often directly related to the proper matching of the mechanical properties of the polymer to those required for the application over the range of temperatures and time scales involved. As a result, the measurement of mechanical properties can provide not only useful design data but also valuable quality control (QC) and NDE information. The following paragraphs describe two of our efforts to utilize this approach for QC and NDE: The first is an ongoing program performed in cooperation with the Naval Research Laboratory and the second is a new effort that is now being developed.

To obtain maximum benefit from mechanical property measurement, it is important that the behavior be characterized over the widest possible ranges of temperatures and time scales (or frequencies). In this regard a device (1) was constructed which combines dynamic mechanical testing and stress relaxation experiments in a single instrument to provide data over an 8-decade time-scale range at temperatures from $-200^{\circ} \mathrm{C}$ to $400^{\circ} \mathrm{C}$. This instrument, which measures torsional shear behavior, includes on-line computerized data acquisition and analysis. It is extremely versatile and has already been used to examine fiber-reinforced composites, structural adhesives $(2,3)$ high-temperature plastics, elastomers, propellants, plastisols, and printing inks. To illustrate the use of this device three particular examples will be briefly discussed.

The problem of quality control for elastomeric materials is a difficult one because many ingredients are used and a number of processing steps are involved. As part of the manufacturing procedure, it is customary to make a number of small samples (ASTM plates) from each batch to serve as material for QC test. Our test device has been adapted so that a small portion of an ASTM plate can be utilized to provide a detailed characterization of the shear properties. For example, Fig. 38 shows a plot of the loss tangent (the ratio of the loss modulus to the storage modulus) as a function of temperature and frequency for a nitrile rubber. For this frequency range, the data were obtained at lower frequencies using an ASTM plate and the superposition technique was then verified and utilized to shift the results to the desired range. With this information an improperly prepared batch can be detected easily and, since the mechanical properties are related to molecular structure and morphology, it is possible in a number of cases to determine what mistakes were made in the formulation.

The nondestructive nature of this measurement technique is particularly helpful when the device is used to follow changes that occur in a material during cure. Two examples of this type have been studied during the past year. The Navy is interested in the properties of certain polyvinyl chloride plastisols for their studies of the interactions between turbulent flow and complaint surfaces. It was found, however, that the properties of this material change as a function of time. Our studies were able to provide a characterization of these properties and their time dependence (Fig. 39). A second example of this type involves inks used by the Bureau of Engraving and Printing for printing 


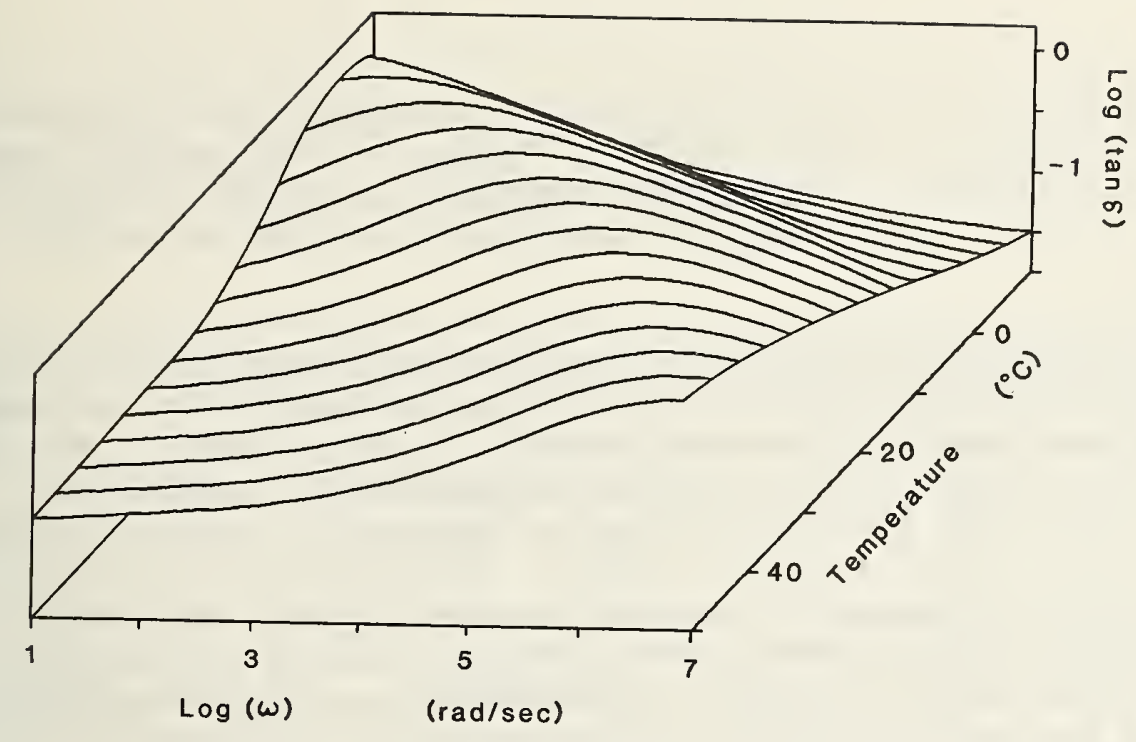

Fig. 38 The loss tangent for a nitrile rubber is plotted against temperature and frequency.

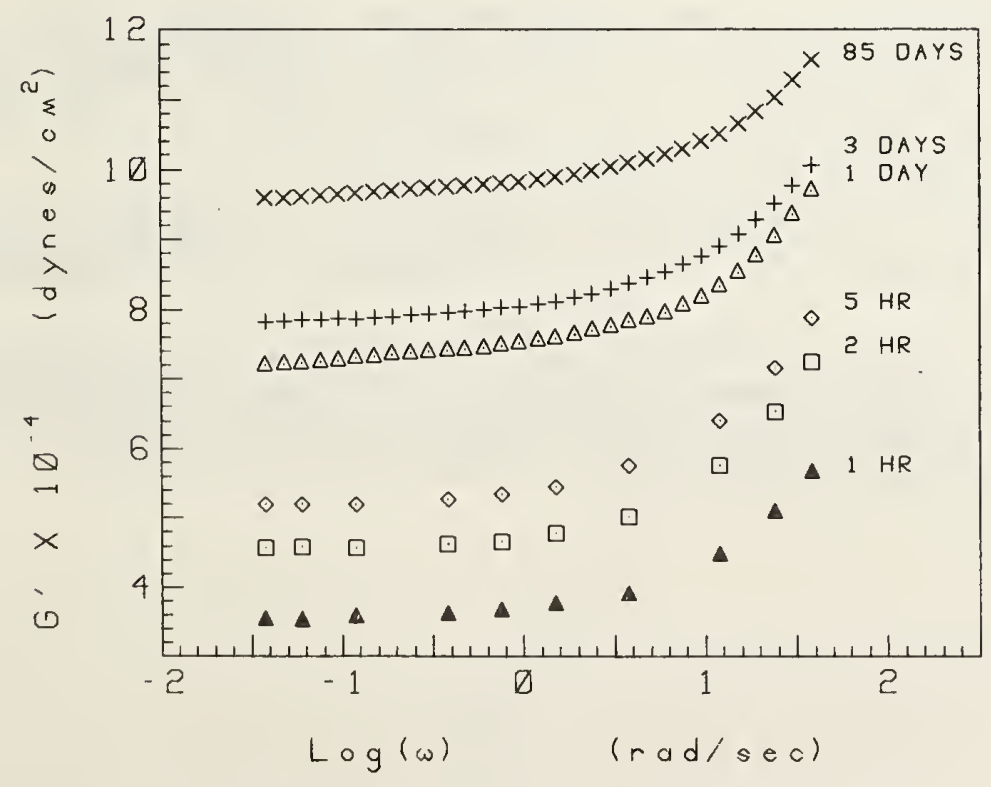

Fig. 39 The dynamic shear modulus for a polyvinyl chloride plastisol is plotted against frequency at various times after preparation: ranging from one hour to 85 days. 
currency. These inks cure during the printing process and good performance can only be obtained when the flow properties change in the required ways at the proper rates during cure. Studies are currently underway to characterize the properties of the inks as a function of cure so that better quality control can be maintained.

In addition to the ongoing studies mentioned above, preparations are being made for work in another area. There are many applications such as adhesives, paints, and coatings that involve thin films. Although thin films are very difficult to study, ultrasonics often represents a useful tool because the attenuation for shear waves can be large enough that the film can be treated as if it were infinitely thick. The device (4) now being set up utilizes a thin strip of aluminum or quartz in which a shear wave can be propagated. When the strip is coated with a film, the wave in the strip generates a shear wave in the coating. Although the wave in the coating cannot be measured directly, it can be characterized by observing how the wave in the strip is altered when the coating is applied. Moreover, if the measurements are made as a function of time, a detailed picture can be obtained for the application, wetting, spreading, consolidation, and hardening of the film. Such information is ideal for optimizing the formulation of polymers for film applications.

\section{References}

1. D. L. Hunston, W. D. Bascom, E. E. Wells, J. D. Fahey, and J. L. Bitner, "Viscoelastic Characterization of Structural Adhesives via Forced Oscillation Experiments," in Adhesion and Adsorption of Polymers, Polymer Science and Technology Series, L. H. Lee, Ed., P. A 321 (P1enum Press, New York, 1980).

2. D. L. Hunston, W. Carter, and J. L. Rushford, "Linear Visco -- Elastic Properties of Solid Polymers as Modeled by a Simple Epoxy," in Developments in Adhesives - 2, A. J. Kinloch, Ed., pp. 125-172. (Applied Science Publishers, Ltd., London, 1981).

3. J. L. Bitner, J. L. Rushford, W. S. Rose, D. L. Hunston, and C. K. Riew, "Viscoelastic Fracture of Structural Adhesives," J. of Adhesion, in press.

4. D. L. Hunston, "Determination of the High Frequency Viscoelastic Properties of Polymers Using Shear Mode Strip Delay Lines," J. Polym. Sci., Pt. C, 35, 201 (1971). 
20. Polymer Transducers

S. Edelman and S. C. Roth

Polymer Science and Standards Division

Center for Materials Science

A method for nondestructive evaluation of the dynamic elastic properties of some kinds of structural elements was worked out during previous years and a paper on the subject was presented at the spring 1980 meeting of the Acoustical Society of America.t The measurement technique made use of the favorable properties of piezoelectric polymer strain gages to measure both axial and shear motion on the same specimen.

During this reporting period, a set of the specialized piezoelectric strain gages required by the method was fabricated. The gages were mounted on a thin-walled PVC cylinder which simulated a structural element under load.

Several measurements were made at different frequencies. The real and the imaginary parts of the dynamic Young's modulus were calculated. Reasonable agreement with published values of the real part was found. The results of two sets of measurements at slightly different frequencies are given in the table below. Each set consists of three nominally identical measurements.

Frequency

$\left(\begin{array}{c}\alpha \\ (m-1)\end{array}\right.$

3.19

3.19

3.19

6836
2.82

2.82

2.83 $\left(m^{\beta}-1\right)$

27.33

27.32

27.33

27.36

27.38

27.38
$E_{1}$
$(\mathrm{~Pa})$

3. $37 \times 10^{9}$

$3.37 \times 10^{9}$

$3.37 \times 10^{9}$

$3.32 \times 10^{9}$

3. $31 \times 10^{9}$

$3.29 \times 10^{9}$
$E_{2}$
$(\mathrm{~Pa})$

$7.97 \times 10^{8}$

$7.99 \times 10^{8}$

$7.99 \times 10^{8}$

$6.91 \times 10^{8}$

$6.92 \times 10^{8}$

$7.45 \times 10^{8}$

$\alpha$ and $\beta$ are the attenuation and phase constants which make up the propagation constant $\quad \gamma=\alpha+\beta$ where $\gamma$ is related to the wave amplitude $\xi_{3}$ by the relation $\xi_{x}=\xi_{0} e^{-\gamma x}$. $E_{1}$ and $E_{2}$ are the real and imaginary parts of the Young's modulus, $E X=E_{I}{ }^{0}+i E_{2}$.

Measurements are presently being made on a section of pipe while the pipe is subjected to a changing tensile stress.

+ Measurement of Dynamic Elastic Moduli;

S. Edelman and N. Newman 
21. Doppler-Broadened Positron Annihilation Lineshape for Detection of Defects in Materials

R. C. Reno* and L. J. Swartzendruber

Metallurgy Division

Center for Materials Science

Positrons that annihilate in a metal produce gamma rays whose energy distributions can be explained in terms of a Doppler shift caused by motion of electrons. When defects are present in the metal, they attract and trap positrons, and alter the Doppler shifts. A measurement of Doppler-broadened annihilation lineshapes thus gives information on the defect nature of materials. Both the annihilation process and the method of detection leave the sample intact, so the technique is extremely useful for the nondestructive evaluation of materials.

Doppler-broadened lineshape measurements are performed by placing a positron source in contact with the sample to be studied. Positrons enter the sample, slow down to thermal energies, and then annihilate with electrons. Each annihilation produces two gamma rays with a total energy equal to the rest mass energy of the positron-electron pair (1022 keV). The distribution of energy between the two gamma rays depends upon the electronic and defect structure of the sample, and is measured with a high resolution solid state detector-multichannel analyzer system. A lineshape parameter, $S$, is defined which is a measure of the inverse width of the distribution.

The addition of a small number of defects to a metal will cause a significant change in the lineshape parameter. This sensitivity is used to observe changes in defect concentration and type. We have been using this technique to study two phenomena: precipitation hardening in aluminum alloys and crystallization of amorphous Pd-Cu-Si.

A commercial aluminum alloy (2219) was subjected to two different thermomechanical heat treatments prior to age hardening. Positron S-parameters were then measured as a function of aging time for each treatment (See Fig. 40). Results indicate that positrons can distinguish between defects surrounding the $\theta$ " and $\theta^{\prime}$ precipitates formed in the two sets of samples.

Figure 41 shows S-parameter data for a Pd-Cu-Si alloy heat treated at various temperatures in the amorphous and crystalline region. At the onset of crystallization, the S-parameter first rises slightly and then gradually decreases. Such behavior can be explained in terms of the generation of defects at grain boundaries during crystallization.

*University of Maryland, Baltimore. 


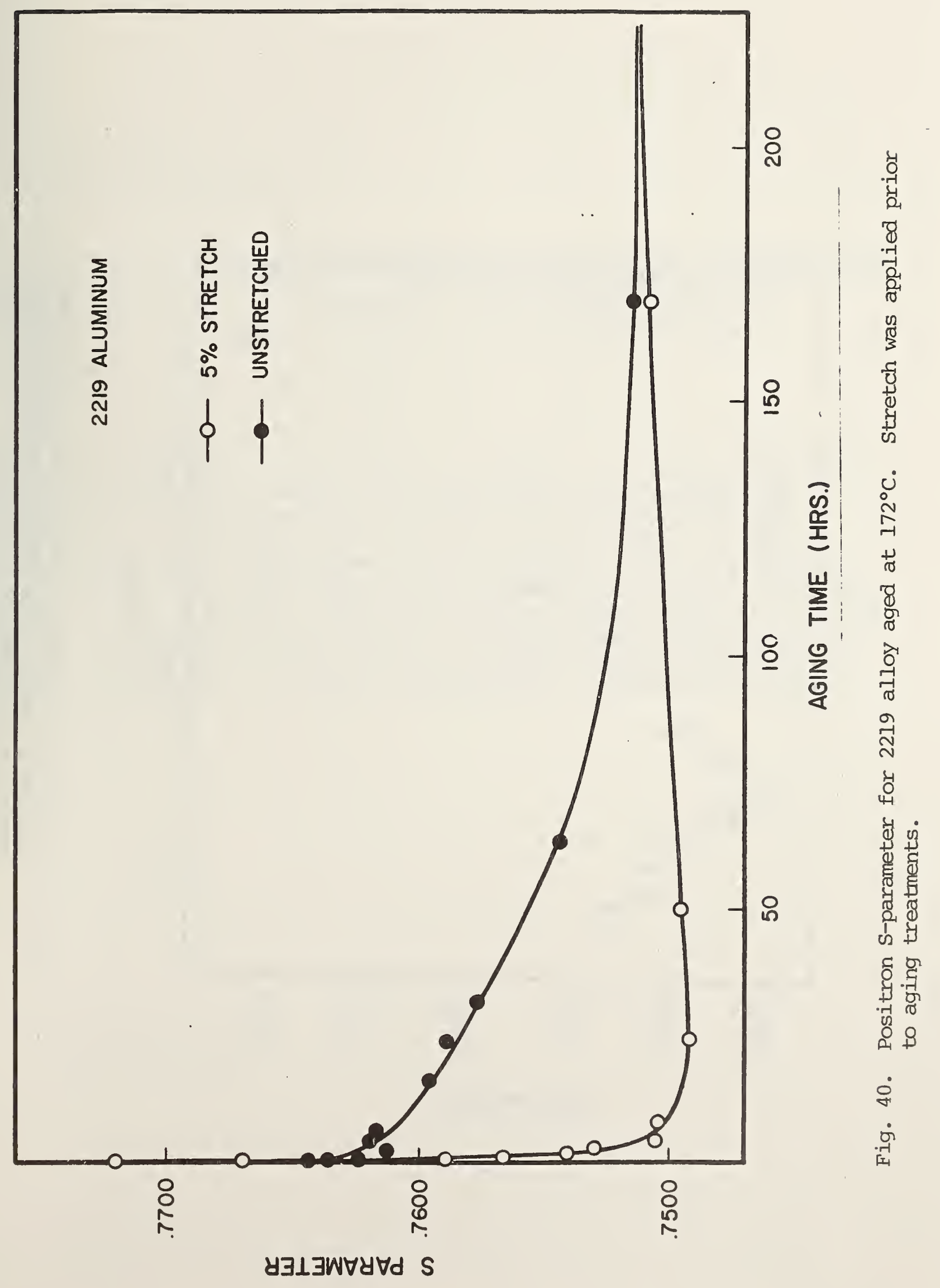




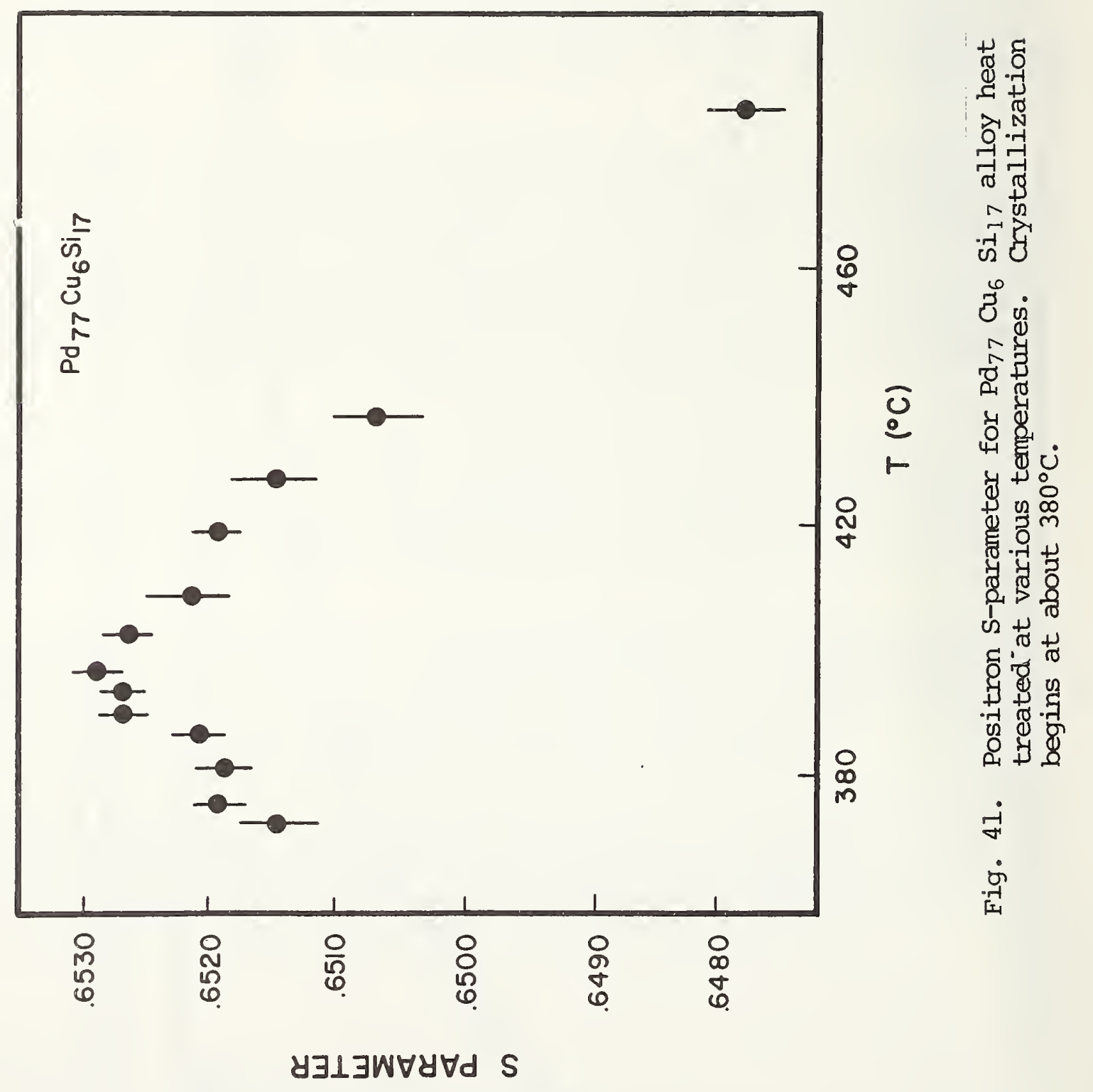


22. Scattering of Acoustic Waves

R. D. Mountain

Thermophysics Division

Center for Chemical Physics

A theoretical study of the scattering of acoustic waves by inhomogeneities in solids has lead to a new way of using scattering data to determine the size and shape of the inhomogeneities. The essential features of the method are embodied in this expression for the scattered power:

$$
\frac{1}{k^{4}} \frac{d P}{d \Omega}=E C_{0}^{2}\left[1-b^{2} \cdot k^{2}+\ldots\right]
$$

Here $k$ is the wavevector of the acoustic radiation, $E$ is a constant involving material and geometrical factors, $C_{0}$ is the volume of the scatterer and $b$ is an effective size parameter of the scatterer which depends on the orientation of the scatterer relative to the scattering angle. Physically, $b$ is a special type of second moment of the scatterer. It is determined by varying the frequency while holding the scattering angle fixed and then fitting the results to this equation. Model calculations indicate that this expression for $d P / d \Omega$ is accurate as long as bk $<1$. The shape of the scatterer can be inferred by determining $b$ for a variety of orientations of the sample being studied. This procedure provides a "second-moment" solution of the inverse scattering problem. 
23. U1trasonic Imaging/Instrumentation

Melvin Linzer and Stephen J. Norton

Signal Processing and Imaging Group

Center for Materials Science

A major breakthrough in ultrasonic imaging was achieved in 1981 . The first exact three-dimensional inverse-scattering solution incorporating broadband illumination and based on variations in both compressibility and density was reported by Norton and Linzer (1). The problem was formulated and solved for three aperture geometries: plane, spherical, and cylindrical. The solutions were obtained under the assumptions of the Born approximation ( $i . e$. , weak scattering) and that only backscattering is measured.

An analysis and computer simulation of a new imaging system for acoustical holography, based on an annular aperture, was also completed (2). Additional work on image reconstruction was carried out in collaboration with T. Sato at the Tokyo Institute of Technology. This included tomographic reconstructions from 1 imited projections using iterative revisions in image and transform spaces $(3,4)$ and a data selection approach for imaging through turbulent media $(5)$.

A theoretical development of perturbation techniques for correcting ultrasonic velocity images has also been carried out. This approach, which does not require ray tracing, is based on a Taylor expansion of the line-integral over the refracted path about the straight line connecting transmitter and receiver. The second term in this expansion is a correction representing the difference in the time delay over the refracted path and the straight path. This correction can then be used to modify the original line-integral measurements, which in turn can be used to reconstruct an improved image corrected for refraction errors. This approach gains little from iteration and, because of its perturbation nature, may be assumed valid for velocity fluctuations within about \pm 5 percent of the mean value, the range of velocity variation in most materials. In fact, computer simulations suggest that good results are possible for even larger variations.

Finally, an analysis of techniques for measuring ultrasonic parameters of materials was made (6), and a manuscript on a sonic technique for detecting interrupted cutting during automated machining was completed (7).

\section{References}

1. S. J. Norton and M. Linzer, "Ultrasonic Reflectivity Imaging in Three Dimensions: Exact Solutions for Plane, Cylindrical, and Spherical Apertures,"(Invited Paper), IEEE Trans. Biomed. Engineering BME-28, 202-220 (1981).

2. S. J. Norton, "Acoustical Holography with an Annular Aperture, J. Acoust. Soc. Amer. (submitted).

3. T. Sato, S. Norton, M. Linzer, 0. Ideda, and M. Hirama, "Tomographic Image Reconstruction from Limited Projections Using Iterative

Revisions in Image and Transform Spaces," App1. Optics 20, 395-399 (1981). 
4. T. Sato, K. Sasaki, Y. Nakamura, M. Linzer, and S. Norton, "Tomographic Image Reconstruction from Limited Projections Using a Coherent Optical Feedback System," Appl. Optics (to be published).

5. T. Sato, M. Linzer, 0. Ikeda, and M. Hirama, "Array Imaging System with Data Selection Processing," J. Acoust. Soc. Amer. 70, September 1981.

6. M. Linzer and S. Norton, "Ultrasonic Tissue Characterization", Ann. Rev. Biophysics and Bioengineering (submitted).

7. F. P. Higgins and M. Linzer, "A Sonic Technique for Detecting Interrupted Cutting During Automated Machining," J. Eng. Ind. (submitted). 
24. Ultrasonic Reference Block Standards and Measurements G. V. Blessing

Mechanical Production Metrology Division

Center for Manufacturing Engineering

Work has continued toward assessing the present limitations and potential improvements of ultrasonic reference block calibration procedures. A set of rogue aluminum blocks, previously observed to yield anomalously high $(600 \%)$ echo amplitude readings, has received further study, including dissection and metallurgical examination. It has been determined that the high readings were caused by radially inhomogeneous aluminum media, rather than improperly drilled flat bottom holes. This was determined by visual inspection of a dissected hole, and amplitude scans of the block without the hole. Further metallurgical studies are planned to determine the exact cause of the material inhomogeneity. 
25. Laboratory Evaluation of U1trasonics for Crack Detection in Concrete L. I. Knab and J. R. Clifton

Structures and Materials Division

Center for Building Technology

A laboratory study was performed to quantify the crack detection capabilities of ultrasonic, through transmission, methods in concrete. Pulse velocity and amplitude measurements were taken perpendicular to the crack plane (in cracked concrete) and parallel to the crack plane (in uncracked concrete). The path length was $152 \mathrm{~mm}$. Parallel crack surfaces, nominally $0.05 \mathrm{~mm}$ apart and having depths of 19,38, and $57 \mathrm{~mm}$ and lengths of $152 \mathrm{~mm}$, were fabricated in the specimens. Transducer frequencies of 150 and $54 \mathrm{kHz}$ were used and specimens were tested at several ages. Concrete with a 28 day compressive strength of about $36 \mathrm{MPa}$ was used.

A "sensitivity" ratio was used to determine if the cracks could be distinguished. The numerator of the "sensitivity" ratio was the difference in velocity of amplitude values between the cracked and uncracked concrete while the denominator represented the inherent batch-to-batch variability of the velocity or amplitude values in uncracked concrete. With the 57 and $38 \mathrm{~mm}$ crack depths, almost all the "sensitivity" values for velocity and amplitude were at least 1.0, indicating that these crack depths could be distinguished under the test conditions. The velocity and amplitude "sensitivity" values with the $19 \mathrm{~mm}$ crack depth were about 0.4 to 0.5 , indicating that the $19 \mathrm{~mm}$ crack depth could not be clearly distinguished. 
26. Reference Samples for U1trasonic Residual Stress Measurement

N. Hsu, T. Proctor, and G. Blessing

Mechanical Production Metrology Division

Center for Manufacturing Engineering

The advantages of using an ultrasonic technique to measure applied or residual stresses in solids are quite appealing. It is nondestructive, it can measure the average bulk stress as well as stresses in the surface layer, and it can resolve biaxial stress states. Furthermore, techniques and instruments are now reasonably well developed and readily available. However, actual field applications of these techniques are still extremely limited.

The difficulties with ultrasonic residual stress measurements have been: inability to determine the initial or zero-stress state, and the presence of texture or texture gradients in most structural metals. In field applications using ultrasonic measurements to determine residual stress, the user usually has a material with both unknown distribution of stress and inhomogenous distributions of crystal grain orientations. With only ultrasonic measurements on the unknown stressed state, the user has to rely on assumed values for reference ultrasonic parameters in order to estimate the residual stress. This procedure causes a large uncertainty which, in turn, leads to questions of the credibility of the ultrasonic technique.

We have initiated the design of a reference sample for ultrasonic residual stress measurement. Our goal is to manufacture a test sample which can be easily reproduced from any structural material of interest and which has a known distribution of stress as well as a zero-stress state. The reference sample is first to be used to establish the reproducibility, the sensitivity, and the resolution of the velocity measurements and then to establish their correlation with other pertinent parameters.

The construction of the sample, a disk, consists of a cylinder shrink-fitted inside a ring and machined flat after fitting. According to the design, the disk has regions in which a zero-stress state or known stress state can be deduced. The sample can be used for both longitudinal and shear wave verification and calibration. Initial experimental results are encouraging. Further improvements and detailed measurements are still in progress. 
27. Nondestructive Evaluation of Nonuniformities in 2024 and 2219 Aluminum Alloy Plate - Relationship to Processing/NASA

L. K. Ives, L. J. Swartzendruber, W. J. Boettinger, M. Rosen, S. D. Ridder, F. S. Biancaniello, R. C. Reno, S. Fick, D. B. Ballard and R. Mehrabian Metallurgy Division

Center for Materials Science

This task was initiated at the National Bureau of Standards at the request of the National Aeronautics and Space Administration shortly after the announcement in August 1979 by the Reynolds Metal Company that about 31 million pounds of aluminum were suspected of containing "soft" spots due to a possible malfunction in the water cooling system at one manufacturing plant. Some of these "soft" spots were reported to be as much as 45 percent below the strength specifications used for the purchase of the aluminum by aircraft manufacturers and suppliers.

The initial concern of this task was the 2219-T87 aluminum alloy used extensively on the space shuttle. The results of our investigations on this alloy were used by the sponsoring agency to ensure that the testing necessary to prove structural integrity had been accomplished prior to launch. The results also had the fortunate effect of expediting the space shuttle launch by el iminating a large amount of unnecessary testing.

The major portion of the work on 2219 has been concluded (1) and attention is now being focused on 2024, an alloy which is more widely used in the construction of commercial aircraft. Because our results established that better nondestructive evaluation techniques are needed for quickly judging the strength of an aluminum plate throughout its thickness rather than simply on the surface as given by hardness and electrical conductivity, on-going investigations of the feasibility of using an ultrasonic technique as an alternative are being carried out on both the 2219 and 2024 alloys.

As with the studies on 2219, the goal of this task is to develop specific relationships between processing variables used during ingot casting, mechanical working, and heat treatment of the 2024 aluminum alloy, and the resulting microstructures, mechanical properties, and NDE responses. This detailed blend of metallurgical examination of the alloy with its NDE response is necessary not only to attack the specific problem at hand but also to gain a fundamental knowledge of the features of metallurgical microstructures which cause changes in NDE parameters. Work in this program is divided into five areas:

1) Studies on as-received plate of 2024 aluminum alloy. This is motivated by the realization that the properties vary through the plate thickness. These variations can have different effects on various NDE techniques.

2) Solidification-Segregation Studies - Part of the variation in properties across finished alloy plate is caused by segregation of the alloy components $(\mathrm{Cu}, \mathrm{Mn})$ during ingot casting. 
3) Determination of time-temperature transformation diagrams (C-curves) and the relationship between mechanical properties and conductivity. Samples produced during various thermomechanical treatments have a wide range of mechanical properties (hardness, yield strength, tensile strength) and conductivity.

4) Electron microscopy studies done on the wide range of metallurgical microstructures produced. Extremely fine $(\sim 0.01 \mathrm{\mu m})$ particles are responsible for the strength of structural aluminum alloys and contribute to associated changes in electrical conductivity.

5) Predictions of heat flow conditions during malfunctions of the commercial process, namely slow quenching from the solution heat treatment temperature; and predictions using experimentally determined C-curves of the resultant mechanical property degradations and changes in NDE response.

In the ultrasonic studies, continuous monitoring of the sound velocity, ultrasonic attenuation, and hardness during the progress of the aging process in 2219 was corroborated with an examination of the specimens by transmission electron microscopy. The experimental data were interpreted within the framework of established theories of diffusional processes and models of precipitation hardening.

The highlights of this investigation were the following:

1. Demonstration of the operational feasibility of an ultrasonic NDE method for monitoring the precipitation process in aluminum alloys in real-time, over a wide temperature interval. Figure 42 shows the variation of the sound velocity (elastic modulus) during the aging process. The minima represent the maximum rate of formation of specific precipitates. Figure 43 shows the changes in the sound wave attenuation (absorption of energy) while the samples undergo the precipitation hardening. The peaks are related to the formation of the particles as well as to the loss of coherency (denoted by 3 ) of the precipitates with the aluminum matrix.

2. Determination of the mechanism and the kinetics of precipitation by simultaneous measurements of changes in sound-wave velocity and attenuation.

3. Calculation of the activation energies and other characteristic parameters related to the formation and growth of precipitates.

4. Elucidation of the relationship between the nature of the precipitates and their specific contribution to strength and hardness of the alloy. In this context the occurrence of attenuation peaks at aging times when hardness peaks are developed, indicating the loss of coherency of the precipitates with the matrix, is noteworthy in Fig. 43. In addition, the effect of prior plastic deformation on the aging process was considered. 
The development of ultrasonic NDE experimental techniques and their use in obtaining the data in this investigation have required research of both a practical and a basic nature. The extension of techniques for ultrasonic NDE to industrial applications requiring on-line, real-time, monitoring during thermomechanical treatment necessitates further development efforts. Improved control of material properties important to the end user offers additional motivation for further study of basic phenomena, e.g., kinetics and mechanism of the aging process in single crystal specimens in order to determine the specific contribution of precipitates located on the prominent crystal planes, and the effects of applied uniaxial and hydrostatic stresses during the precipitation process.

The investigation of 2024 will be completed during Fiscal Year 1982 when a final report on this alloy will be issued. The large amount of data and the techniques developed are expected to be of value for improving both the nondestructive evaluation of the finished product and the processing of material.

\section{Reference}

1. "NBS: Nondestructive Evaluation of Nonuniformities in 2219 Aluminum Alloy Plate - Relationship to processing", NBSIR 80-2069, December 1980.

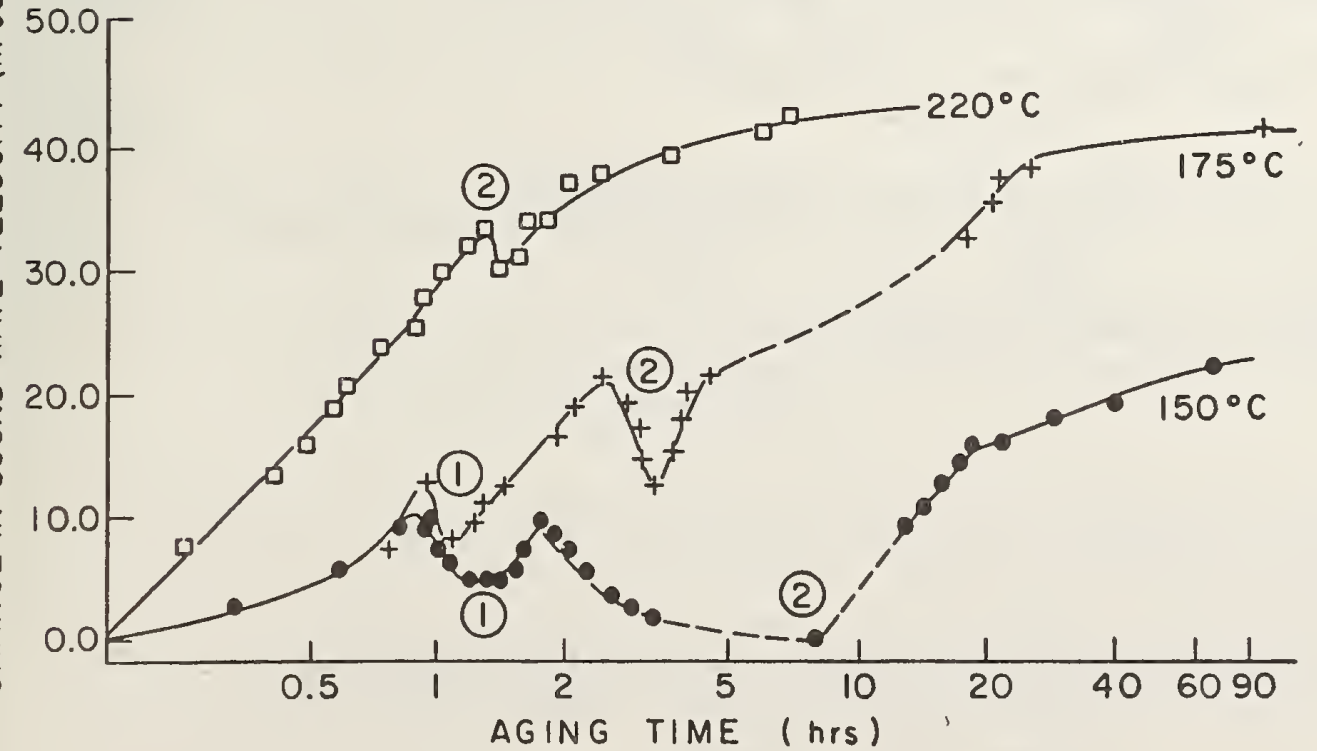

Fig. 42 Change in uitrasonic sound wave velocity as a function of aging time at three different temperatures in 2219 a luminum alloy. 


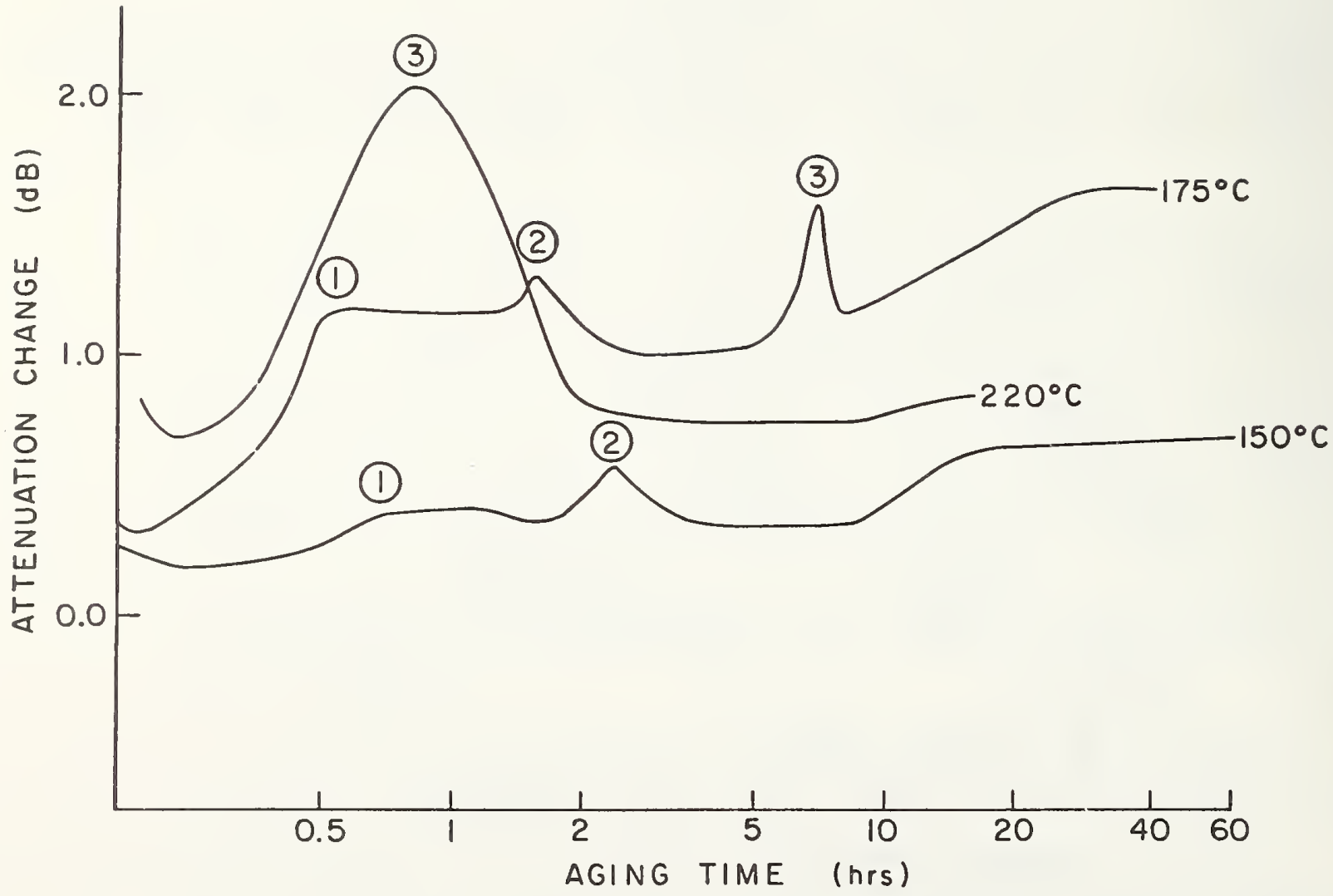

Fig. 43 Change in ultrasonic sound wave attenuation as a function of aging time at three different temperatures in 2279 a luminum alloy. 
28. Safety Factors and Mathematical Modeling

J. T. Fong

Mathematical Analysis Division

Center for Applied Mathematics

Engineering decision-making is characterized by two information-gathering processes:

(a) Mathematical modeling of the performance of a component or system using a combination of theoretical results and experimental data.

(b) Application of so-called "engineering judgment" to cover uncertainties due to a lack of information on all aspects of an engineering problem.

The first process is, in general, quantitative, while the second is qualitative by tradition and necessity except that at some point along the decision-making process, a numerical quantity known as a safety factor is usually employed.

For engineering decisions involving public safety or the protection of the environment, the traditional process of choosing a safety factor by committee or peer review is vulnerable to the charge that the judgment factor has no rational basis. The issue arose in the summer of 1976 when the National Bureau of Standards was asked by the U.S. Department of Transportation to assess the integrity of about 600 welds of the Trans-Alaska 0il Pipeline which were known to contain defects larger than allowable under the federal regulations.

The technical results of the 1976 NBS study consisted of two distinct parts:

(a) Fracture mechanics analysis for the generation of allowable defect size curves was found acceptable as a basis for a regulatory decision.

(b) Defect size estimates from field radiographs with insufficient control on process parameters were found unreliable to contribute to a technical assessment.

An attempt to introduce a safety factor on the defect sizes was rejected because there was no framework to introduce engineering judgment either by a committee of experts or by a theoretical model. The lack of a positive technical result on defect dimensioning contributed, as believed by this author, to the final ruling of the regulator that all defective welds except three under Koyukuk River had to be repaired to assure the structural integrity of the pipeline.

Aside from the economical and political aspects of the regulatory decision, the 1976 NBS study revealed a serious technical deficiency in the application of NDE data. With all the training, application, and experience behind the NDE industry, why should three reputable inspection or auditing teams disagree on their defect size estimates by factors ranging from 1 to 7 ? If human factors were critical in the outcome of an NDE call, why didn't the NDE industry initiate a research and development program to characterize this source of uncertainty? If NDE was intended to be a critical element in engineering and regulatory decision making, why was the subject seldom included as a required course in an engineering curriculum? 
To answer these questions, this project was initiated in FY78 with some preliminary results reported in the 7980 Annual NDE Report. The following is a summary of new results developed in FY81 and documented in a manuscript entitled "Safety Factor on Defect Sizes: A Combined Statistical and Engineering Approach".

It is well known in the statistics literature that confidence interval estimates for the true mean of a certain measured quantity of a population can be made from knowledge of (a) the sample size, (b) the sample mean, and (c) either the sample variance or an estimate of the true variance based on previous measurements. Based on the notation used in an NBS publication (Experimental Statistics, by M. G. Natrella, NBS Handbook 91, 1963), the one-sided upper bound $X_{U}^{\prime}$ for a $100(1-\alpha) \%$ confidence interval for the true mean of a population is given by either of the following two formulas:

$$
\begin{array}{ll}
X_{U}^{\prime}=\bar{X}+t \frac{s}{\sqrt{n}} \quad \text { (if the sample variance is used). } \\
X_{U}^{\prime}=\bar{X}+z \frac{\sigma}{\sqrt{n}} \quad \text { (if the true variance is used). }
\end{array}
$$

In equations (1) and (2), $\bar{X}$ denotes the sample mean, $n$, the sample size, $s^{2}$, the sample variance, $\sigma^{2}$, the estimated true variance, $t$, the tabulated value of a t-distribution for $100(1-\alpha) \%$ confidence and $n-1$ degrees of freedom, and $z$, the tabulated value of a $z$-distribution for $100(1-\alpha) \%$ confidence and $n-1$ degrees of freedom.

Based on a method first introduced by Mandel ("Analysis of Interlaboratory Test Data," ASTM Standardization News, Vo1. 5, No. 3, p. 17, 1977), multi-team inspection data on a defect dimension of $n$ welds are analyzed as if $m$ replicas of measurements were made on each weld. Here $m$ is the number of teams inspecting the welds. The variance decomposition formula used by Mandel (1977) can be used here to provide an estimate of the reproducibility from one defect to another (within-the-weld population) and the repeatability from one team to another (between-team) of the inspection process. Computational formulas are omitted here for brevity except that the decomposition formula with the appropriate change of notation can be exhibited to show the simplicity of the approach:

$$
\sigma^{2}(\bar{x})=\sigma^{2}(n)+(1 / m) \sigma^{2}(m),
$$

where $\sigma^{2}(\bar{X})$ denotes the variance of the mean, $\sigma_{(m)}^{2}$, the between-team variance which conceivably can include variabilities due to both the measurement process and human factors, and $\sigma_{(n)}$, the within-the-weld-population variance which should indicate variabilities due to the welding process and the associated human factors. Naturally, for a given set of NDE data, we can only estimate those variances by computing $s^{2}(\bar{X}), s^{2}(m)$, and $s^{2}(n)$. We are, however, not obligated to use those computed values if our experience on related applications 
indicates that the true variances may be much higher. What we are proposing, therefore, is a combination of statistical analysis and engineering judgment in arriving at a formula for a safety factor for defect sizes:

$$
S . F ._{(n \times m)}=\frac{\left\{\bar{X}_{(n)}+t s(\bar{X}) / \sqrt{ } n\right\}+z\left\{\sigma_{(m)}^{2}+\sigma^{2}(n)^{\frac{1 / 2}{2}}\right.}{\bar{X}_{(n)}} \text {, }
$$

where S. F. denotes a safety factor based on a set of data on $n$ welds by $m$ inspecting teams, and $\bar{X}_{(p)}$ is the grand sample mean obtained by first calculating the mean of $m$ replicates and then computing the mean of averages from the $n$ welds. Engineering judgment is introduced through the use of ${ }^{\sigma ?}(m)$ and $\sigma^{2}(n)$. Additional formulas are used to guide the selection of those

variances based on estimated defect sizes. It is worth noting that the terms in the first curved brackets on the right of equation (4) give an upper bound estimate of the true mean of the population, whereas the terms in the second curved brackets bring together an engineer's total knowledge on the variability of the measurement and welding processes including those due to human factors.

Clearly, the computed safety factor would vary according to the level of confidence one prescribes for the problem at hand. To illustrate this point, numerical results based on length of elongated slag data of ten welds (\#81135, $81180,81293,81397,81401,81411,81420,81424,81451$, and 81474) of the Trans-Alaska 011 Pipeline, as documented in the 1976 NBS study report, indicate that for three levels of confidence such as 99\%, 95\%, and $90 \%$, the corresponding safety factors decrease from 2.71 (for 99\%), through $2.19(95 \%)$, to $1.92(90 \%)$.

This approach describes a framework for constructing a safety factor for defect sizes based on a multi-team inspection or auditing of welds in a critical structure. This framework combines rigorous statistical techniques with engineering judgment, and is particularly valuable for dealing with measurement processes where the human factor is a significant contributor to the process variability. This work was presented at an ASME-ASNT joint symposium on NDE: Reliability and Human Factors, Oct. 12-13, 1981, Atlanta, Ga. 
29. Visual Acuity Requirements for Nondestructive Testing.

G. Yonemura

Environmental Design Research Division

Center for Building Technology

National Engineering Laboratory

Quantitative measures describing the physical correlates of what the eye is expected to detect should be the basis for designing the visual acuity test targets for nondestructive testing. In radiographic inspections, dimensional descriptions of the defect measured on the material have limited value. The inspector is asked to look at the radiograph, consequently the physical measure of interest for visual acuity testing is the defect as displayed on the $f i 1 \mathrm{~m}$, regardless of how much it may differ from the actual defect.

Based on microdensitrometric scans of sample radiographs and supporting data from the literature, the recommended parameters are summarized in Table $V$. The optotype should be a thin line darker than the background. The background Tuminance of the test target is kept constant at $85+5$ nit $(25+1.5$ $\mathrm{ftL})$. Three contrast 1 evels are recommended. The 1 ine widths $\vec{r}$ ecommended are $0.75,1.0$ and 1.5 minute of arc. The length of the 1 ines are kept constant at 107 minute of arc. These angular measures are based on a viewing distance of $40 \mathrm{~cm}(16 \mathrm{in})$. Two levels of line sharpness are recommended--one with a sharp edge that will be similar to most optotypes used in visual acuity testing, and one with a blurred edge conforming to real-world radiographs.

Samples of the reference test targets are presented in Fig. 44. The testees are asked to give the orientation of the 1 ine. These targets may be used for periodic tests by the employer or for more frequent self testing by the inspector himself.

Background materials and supporting literature are discussed in NBS Tech Note 1143, Visual Acuity Testing of Radiographic Inspectors in Nondestructive Inspection. This document will serve as a core from which a consensus document will be developed for the military after discussions with the health departments and radiographic nondestructive installations in the military. 
Table V. Visual Acuity Test Parameters

$$
\begin{array}{ll}
\text { Variable } & \text { Number of } \\
\text { Conditions }
\end{array}
$$

Figure-Ground

Background Luminance (nit/ftL)

Contrast

Line Width (minutes)

Line Length (minutes)

Viewing Distance $(\mathrm{cm} / \mathrm{in})$

Blur

Line Orientation

Light Source (viewer)

Total (combinations)
1

1

3

3

1

1

2

4

1

72
Conditions

Dark on light

$85 \% 5 / 25 \% 1.5$

$0.1,0.3,0.85$

$0.75,1.0,1.5$

107

$40 / 16$

Sharp, blurred

Perpendicular/horizontal oblique right/left

Incandescent, fluorescent 
FRONT

Yertical

Contrast $\underline{0.85}$

Sharp
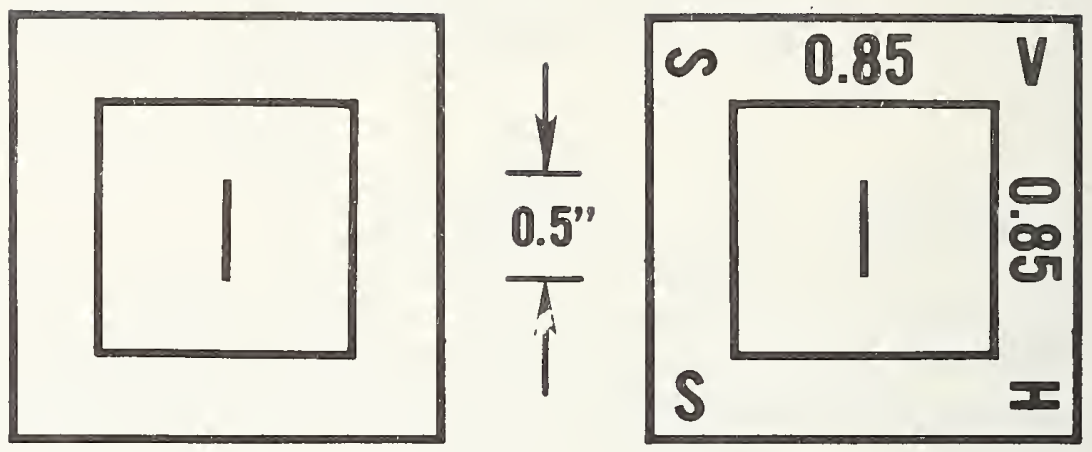

Rotated $90^{\circ}$

Horizontal

Contrast $\underline{0.85}$

Sharp
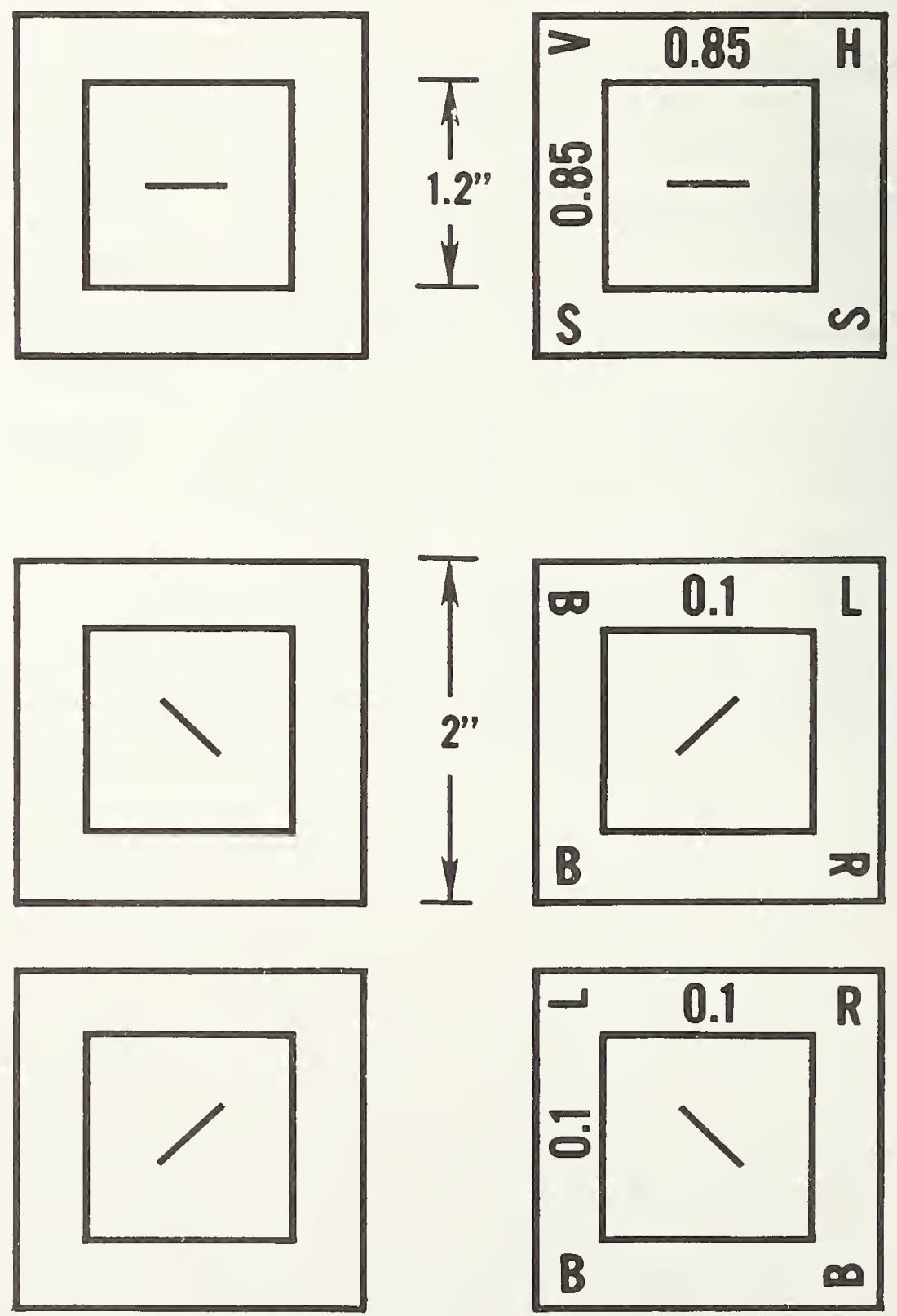

Figure 44. Examples of acuity tests showing line orientation and dimensions. 
30. Wear Condition Monitoring

A. W. Ruff

Metallurgy Division

Center for Materials Science

This project is concerned with the problem of monitoring the condition of operating mechanical equipment, particularly its wear. In many cases involving rotating machinery, e.g., turbines, pumps, and motors, wear is the principal 1 ife-limiting process of degredation. A number of different methods are used to condition-monitor such systems. These include thermal measurements, vibration measurements, dimensional measurements and lubricant analysis. This project is addressing measurement and standard needs involving two particular techniques: (1) wear debris recovery and analysis, and (2) acoustic emission detection and analysis. Progress has been made this year in both areas and is described below.

Many operating mechanical systems that are subject to wear failures are serviced by built-in lubrication systems. Techniques exist to determine the condition of the lubricant using chemical analysis methods that will indicate lubricant failure. However, it is frequently found that chemical analysis does not give a sufficiently early warning of failure. A more direct approach involves recovering the wear debris particles themselves from the lubricant fluid. Analysis of the wear particle size, type, and amount can provide both early and detailed information on the wear process involved, and the rate at which wear is taking place. For example, in a rotating bearing system, it is normal to observe the moderate generation of mid-sized wear particles initially as the system begins to operate from the new $7 y$ manufactured condition. After this a low generation rate of small particles is found throughout the usual operating life. Finally, a high generation rate of large particles is noted that corresponds to the onset of rapid wear at the end of useful 1ife. Wear debris analysis methods can determine all of these stages and in many cases can provide early indication of the onset of final high wear and failure. Chemical analysis methods cannot provide this degree of information.

This project has been concerned with a magnetic method of recovery and analysis of wear debris particles from lubricating oil. This method, termed ferrography, can remove virtually all metal or metallic oxide particles from the $0 i l$ and precipitate the particles according to size on a glass substrate. This "ferrogram" is very convenient for further microscopic study and quantitative measurement of the wear particles. The density of particles can be measured optically and can be proportional to the amount (or rate) of wear in the system. This project has contributed significantly in past years to the techniques of ferrography, in particular, to the analys is of wear particle type and size, and their relation to worn surface damage. In the past year, the project has emphasized standards needs in ferrography. A group of six laboratories in the U.S. (including NBS) have been conducting interlaboratory test comparisons (round-robins) for two years, and considerable progress has now been made. The last measurement cycle involved three samples of wear debris in 0i1: one produced at NBS by two methods of wear in a way that we expect is reproducible, and two obtained from field operating 
systems. Both oil samples and pre-prepared ferrograms were circulated among the 6 laboratories. Typical precision of measurement was about $5 \%$ coefficient of variation ( $\mathrm{CV}$ ) on wear particle density at any location on the ferrogram. However, the additional interlaboratory cv was about $40 \%$. The problem was traced to the optical density measuring equipment used. It was clear that each lab's instrument was operating differently and that proper standardization of that step should reduce the disagreement considerably. The method for preparing the ferrogram (recovering the debris particles and depositing them according to size) was found reproducible and satisfactory. This particular method is now being sent through an SAE committee on standards for official U.S. approval. After over 5 years of development, largely through Navy sponsored work, that standard should greatly improve the wear debris analys is capability of any properly trained and equipped laboratory.

The remaining problem of interlaboratory error is best handled by the development of standard reference materials. Two different materials are needed. One will serve as a "standard" ferrogram; this will provide the instrument operator with a means of calibrating his locating system, aperature system, and general microscope operation. The second will provide 4 or 5 levels for calibrating particle density from 0 to $100 \%$. Together, these reference materials should enable reproducible measurement of wear particle density to about $10 \%$ to $15 \%$, thus putting the entire method on a firm quantitative basis. These two developments should be complete in the next year.

The second effort in this project is concerned with applying techniques of acoustic emission detection to the wear problem. This approach could be valuable in applications where sudden wear failure of a critical component, e.g., a gas turbine shaft bearing, cannot be tolerated. Sudden failure in rolling bearings usually results from subsurface crack nucleation and slow crack growth, over lengthy periods of operation. One crack will eventually reach the surface, releasing a large wear fragment (spalling). The bearing surface is then badly damaged and the process accelerates until total bearing failure occurs. It would be extremely valuable to have techniques to detect the development of spalling failure early enough to avoid the catastrophic stage. Since the growth of the subsurface crack occurs incrementally and slowly, acoustic emission monitoring of crack growth, involving bursts of energy release, appears feasible. A laboratory wear test system has been instrumented to carry out simple experiments to attempt such detection and identification. The system involves a block-on-ring test geometry with the block specimen undergoing cyclic wear and steady sliding wear. The sliding wear background consists of random noise events associated with surface friction and oxide rubbing processes. The cyclic wear component is intended to lead to slow growth of preexisting cracks beneath the wear surface. The detection system consists of an adjustable 60-710 db low noise amplifier, high frequency and low frequency filtering, a conventional AE transducer detector, and a modified video tape recorder. Wear experiments are carried out during which continual recording of the detected acoustic signals takes place. The recorded signals can be monitored on an oscilloscope and a stop action feature permits inspection of any portion of the recorded waveforms. 
The problems being attacked first include the choice of test material, and the characteristics of the repetitive machine noise and the sliding wear background noise. Several different steel blocks have been explored, including tool steels and plain carbon steels. It was found difficult to pre-establish proper crack growth conditions in those steels. Current efforts are on temper embrittled steels where the crack growth characteristics of repetitive machine noise have been identified in several cases; however, the background surface rubbing noise may prove more of an interference to crack growth detection. Our plan calls for the use of AE signature detection methods being developed by R. Clough and J. Simmons, using a digital signal processing approach. We plan to play back the VTR signals into their processing system and identify the crack-specific information. Some results using this particular approach should be obtained next year. 
31. X-Radiography

R. Placious, D. Polansky, C. Dick, and J. Motz

Radiation Physics Division

Center for Radiation Research

This report describes the NDE activities of the NBS X-Ray Physics Group pertaining to industrial radiography. The general goal of this program is to provide assistance to groups involved in industrial radiographic inspection by developing and making available improved standards, procedures, measurements, and imaging systems. This program is described by the following projects in industrial radiography, which include the names of the principal investigators.

1. Standard Method for Measuring the Image Quality Response of

Industrial Radiographic Film (R. C. Placious and D. Polansky).

This standard method required the development of a test object which is now available from the NBS SRM Office. This standard method was developed for low-energy $(200-k V)$ use, and it will appear in the next printing of the ASTM Book of Standards.

\section{High-Energy Image Quality Measurements (D. Polansky and R. Placious)}

The completion of the low-energy standard method mentioned above generated interest in producing a standard which would perform essentially the same function at high photon energies (cobalt-60 and industrial Linacs up to $15 \mathrm{MeV}$ ). The NBS X-Ray Physics Group has undertaken the responsibility of designing and fabricating the high-energy test object, and then of arranging for round robin tests among various industrial laboratories, and of coordinating all the resulting test data. At the present time, the equivalent penetrameter sensitivity is the basis for developing the high-energy standard method. Present results indicate that the latest test object design can be successfully used as a standard to distinguish between films in a specified radiographic system. In order to obtain statistical data, the radiographs are being evaluated at various reader stations throughout the country.

\section{Radiographic Film Storage and Processing (R. C. Placious)}

Another activity within ASTM which has been assumed by NBS is the responsibility for developing appropriate standards for radiographic film storage and processing. A draft for film processing has been developed and is being reviewed by appropriate experts. Another draft will be developed for film storage. Both drafts will eventually be ASTM standards and will supplant the material now included in ASTM standard E-94-77.

\section{Unsharpness Measurements (D. Polansky)}

There were two approaches to possible methods of measuring unsharpness that were investigated. First, the optical density variations at the extremities of a density edge trace were measured. These measurements were not reproducible since the automatic scanning densitometer was too sensitive to local density 
variations. Further investigation is required in order to determine whether this first approach is viable. In the second approach, the visibility limit of the edges of a stepped slit was investigated. Using conventional radiographic techniques it was determined that the visibility limit of film types $A A$ and $T$ were equal. Therefore, this second approach was not satisfactory because one could not differentiate between two industrial fiims that are considered to produce radiography of different quality.

At the present time unsharpness measurements are made with the "CERL" indicators developed at the Central Electronics Research Laboratory. A detailed evaluation of the CERL indicator for this purpose will be made at NBS in the near future.

5. Radiographic Reference Standards for Pipeline Weld Flays (Welding Research Council) (R. C. Placious)

The goal of this work is to produce a reference standard or standards for assessing, under field conditions, the volume content or projected area of three dimensional flaws in welds. Such reference devices would reduce considerably the error in the reader's estimate of the flaw content. These estimates are presently based primarily on the reader's judgment and not on direct measurement. Preliminary designs may take the form of overlays or radiographs of known flaw content.

\section{Applications of Fluorescent Screens to Industrial Radiography (C. Dick)}

Real time imaging systems now under development for on-line inspection require the use of an $x$-ray fluorescent screen to convert the $x$-ray fluence to a wavelength suitable for the electro-optical imaging devices being employed. In this program, a method is developed to experimentally evaluate the image information transfer properties of $x$-ray fluorescent screens for real time radiography. Measurements have been made of the absolute $x$-ray detection efficiency and of the number of optical photons emitted from the screen as a function of the incident $x$-ray energy. Data have been obtained which show the number of light photons emitted per absorbed $x$-ray for a calcium tungstate and a rare earth oxysulfide screen as a function of the incident $x$-ray energy from 20 to $70 \mathrm{keV}$, and which give the values of the $x$-ray detection efficiency and the detective quantum efficiency for the above screens. These measurements are being extended to approximately $500 \mathrm{keV}$ and will thus encompass the range used in industrial NDE. In addition, preliminary experiments are underway to determine the energy conversion mechanism of metallic and fluoro-metallic screens which are used at these higher energies. 
32. Comparison of Non-Reactor Based Neutron Radiographic Facilities

W. L. Parker and D. A. Garrett

Reactor Radiation Division

Center for Materials Science

The objective of these investigations was to obtain a comparison of the characteristics of three major thermal neutron radiographic facilities which did not utilize a nuclear reactor as a source of neutrons. In the evaluation of each facility, the radiographic results were quantified as closely as possible utilizing results obtained with the National Bureau of Standards Research Reactor (NBSR) as benchmarks.

The three facilities evaluated were:

a) The subcritical assembly at the Mound Research Laboratories, Miamisburg, Ohio.

b) The $2.5 \mathrm{MeV}$ Van de Graaff accelerator facility at the Pantex Plant of the Silas Mason Hanger, Silas Mason Co. in Amarillo, Texas.

c) The Crockroft-Walton accelerator-based system at the Advanced Technology Center of the Vought Corporation, Dallas, Texas.

In all cases, the primary neutrons possessed energies greater than thermal energies, i.e., greater than $0.025 \mathrm{eV}$. It was necessary to thermalize the primary neutrons by the process of inelastic scattering in a moderator such as light water, graphite, or other low atomic number scattering medium.

For each neutron source, measurements were made of the $L / D$ ratio at varying distances from the aperture, and the size and position of the effective aperture were determined. The ratio of the film darkening due to gamma ray beam contamination to that due to the thermal neutron flux was used as a meaningful measure of the $n_{\gamma} / n_{T}$ ratio. The flux and cadmium ratio were not measured during the evaluation, but are given as reported from previous measurements.

The subcritical assembly consists of a multiplied ${ }^{252} \mathrm{Cf}$ spontaneous fission neutron source and produces a total primary fission neutron yield of approximately $5.8 \times 10^{10} \mathrm{n}-\mathrm{sec}^{-1}$.

The neutron yield from the ${ }^{252} \mathrm{Cf}$ source is multiplied by a factor of 28 in a ${ }^{235} U$ subcritical assembly having a $k$ effective of 0.990 . Primary neutron moderation is achieved with light water. Assembly multiplication is controlled by cadmium poison rods.

The 2.5 MeV Van de Graaff accelerator is employed to accelerate a deuteron beam onto a beryllium target. Neutrons having peaks at well defined energies are produced in the energy range between $2 \mathrm{MeV}$ and $5 \mathrm{MeV}$. The primary neutron yield is based on the ${ }^{9} \mathrm{Be}\left({ }^{2} \mathrm{H}, \mathrm{n}\right){ }^{10_{\mathrm{B}}}$ reaction and is controllable by varying both the accelerating potential and ${ }^{2} \mathrm{H}$ beam current striking the Be target. The target is placed at the center of a spherical steel tank and neutron moderation achieved by inelastic scattering interactions with the $H$ component of 1 ight water. 
The Vought facility is based on neutron production utilizing a Cockroft-Walton accelerator. Deuterons are accelerated to $185 \mathrm{keV}$ and allowed to strike a tritium target. Primary neutrons having a well defined energy peak at $14 \mathrm{MeV}$ are produced, based on the ${ }^{3} \mathrm{H}\left({ }^{2} \mathrm{H}, n\right)^{4} \mathrm{He}$ reaction. The Cockroft-Walton accelerator is a commercially-available sealed-tube unit and produces a total primary neutron yield of $2 \times 10^{11} n-\mathrm{sec}^{-1}$. The target is placed at the center of a three-foot diameter spherical tank. Neutron moderation is achieved by inelastic scattering interactions with the hydrogen and carbon components contained in the moderator medium.

The comparative data obtained as a result of this evaluation program are given in Table VI. In all cases, the system characteristics were determined with facility parameters that are employed under normal production operating conditions.

During the course of these investigations, every effort was made to keep the data-gathering process as uniform as possible for each system. The conclusions are based on the system data gathered, past system performance history, and hands-on experience. These conclusions are:

Subcritical Assembly

- May be left unattended during operation

- Low maintenance costs

- Capable of high resolution performance

- Large, heavy, costly

- ${ }^{252}$ Cf spontaneous fission neutron source constantly decays with a half-life of 2.3 years

- Fixed installation

- Downtime less than 10 percent

Van de Graaff Accelerator

- Does not require a primary neutron source which constantly decays

- Shielding requirements less stringent than those required for the subcritical assembly

- Downtime less than 10 percent

- Fixed installation

- Present units require constant operation attendance

- Intermediate cost

\section{Cockroft-Walton Accelerator}

- Transportable

- Low cost

- High reliability with negligible maintenance costs

- Beam rotation through $180^{\circ}$

- Present systems require operator attendance during operation

- Low neutron production rate

- High operating cost due to tube replacement at end of warranty period

- Negligible downtime and maintenance costs 


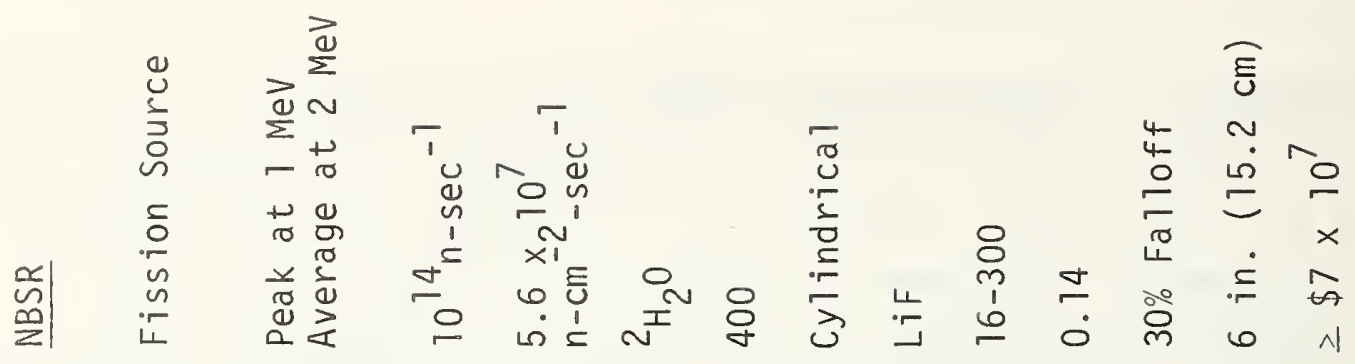

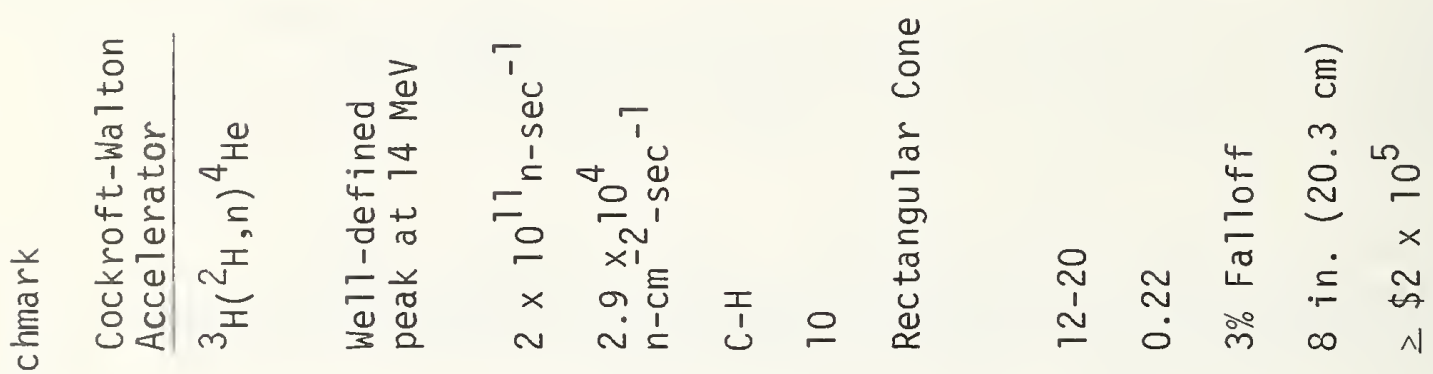

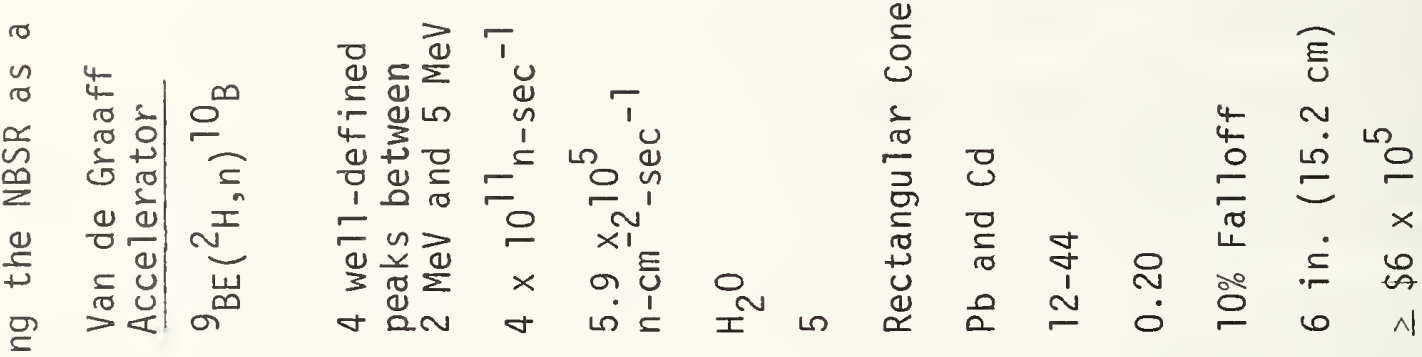

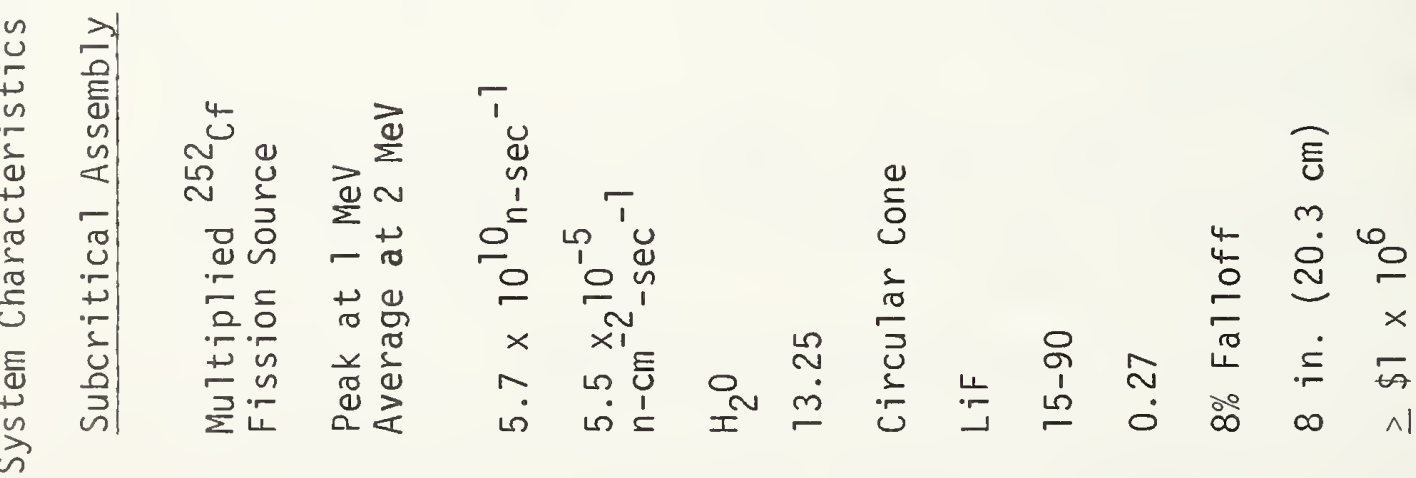

品

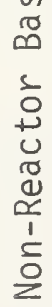

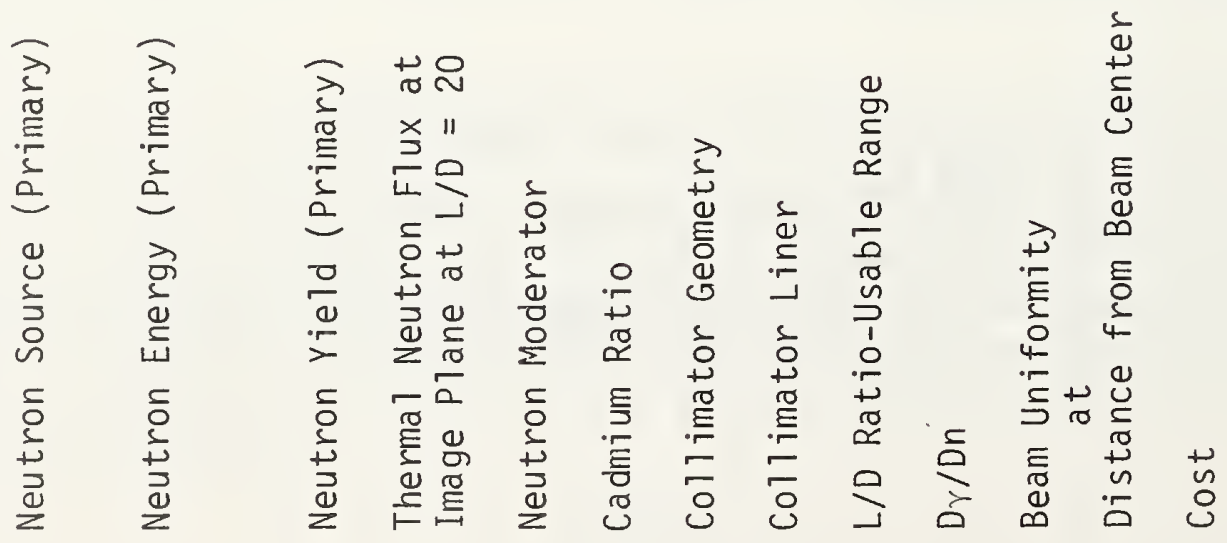


33. X-Ray Residual Stress Mapping in Industrial Materials by Energy Dispersive Diffractometry

M. Kuriyama, W. Boettinger and C. Bechtoldt

Metallurgy Division

Center for Materials Science

This project is part of the Metallurgy Division's participation in the NBS NDE program. The objective is to develop a nondestructive method of mapping the distribution of residual stresses within industrial materials of considerable thickness. This has been carried out in collaboration with Robert Placious, Radiation Physics Division, Center for Radiation Research.

Improved quality control of industrial materials requires quantitative information concerning the stress distribution near cracks under load and residual stress distributions after different types of cold work and heat treatment. This information enhances our understanding of the crack formation and subsequent propagation inside materials, and leads to a way to produce better materials in industry. For monitoring the structural integrity of, for example, weld zones, quantitative information is required for the strain distribution near the weld zone including the heat-affected zone as well as the fusion zone. The most accurate current method for the determination of residual stresses uses $x$-ray Bragg diffractometry from the surface of materials (1). Although the accuracy is far beyond any other techniques, this technique is unfortunately incapable of detecting stresses in the interior of bulk materials $(>1 \mathrm{~mm})$.

Several years ago, we proposed (2) an entirely different approach to the $x$-ray evaluation of residual strains (or stresses) although it also uses the principles of $x$-ray diffraction. This approach uses energy dispersive spectroscopy, where an energy dispersive solid state detector is employed with a we11-collimated beam of high-energy photons. In energy dispersive spectroscopy, one is concerned with energy spectral profiles and peak positions at different energy values, instead of Bragg angles which play a key role in Bragg diffractometry. These Bragg energy peak positions represent the lattice constants in a local volume, and herice local strains. We proved, using ordinary laboratory $x$-ray sources and thin materials, that the desired resolution and detectability can be achieved for the determination of residual stresses if this technique is employed simultaneously with a curve fitting technique (3). This year we demonstrated a similar capability using an industrial (250 kV) radiographic $x$-ray source for commercial steel plates up to $3 \mathrm{~cm}$ (or more than one inch) thick. The distribution of stress can be mapped across the plates with a we11-collimated incident beam and a well-defined detector opening.

In this method, well-defined diffraction energy peaks should be obtained at energies of $100 \mathrm{keV}$ or more, so that penetration of a thick material is possible and can determine the stress within the bulk material. These peaks should have a Gaussian profile so that the curve-fitting technique can be used for the determination of the centroid (or peak) positions with the accuracy increased by a factor of 100 over the resolution limit of solid state detectors. All of these principles were confirmed previously (3) with thin materials using 
low-energy photons. This year's objective is to confirm these points using high-energy photons with thick materiais. Figure 45 shows the variation of strains (and, in turn, stresses) across a weld zone measured by this proposed technique in one of the Alaskan pipe lines $(4,5)$. The material is $14.6 \mathrm{~mm}$ thick and has a minimum welded zone $6 \mathrm{~mm}$ wide centered at position 0 . The statistical error ( $\sigma$-value) in this measurement is less than $1 \times 10^{-4}$ in strain and $3000 \mathrm{psi}$ in stress. This indicates the detectability of stress. Reproducibility was also confirmed. In this example the counting times were only moderate. With longer counting times or an increased photon flux, the counting statistics can be improved. Using laboratory $x$-ray sources up to $40 \mathrm{kV}$, strains of $5 \times 10^{-5}$ (300 psi) can be easily detected with reliable reproducibility and good counting statistics. Thus, the present demonstration of the expanded capability to higher energy has shown a potentially powerful scientific tool to study and characterize flaws and microstructure with great accuracy; for example, weld zones can be studied across the zones through the depth.

\section{References}

1. J. B. Cohen, H. Dolle, and M. E. James, "Stress Analysis from Powder Diffraction Patterns," Proceedings of a Symposium on Accuracy in Powder Diffraction, NBS Special Publ. 567, p. 453 (1980).

2. M. Kuriyama, W. J. Boettinger, and H. E. Burdette, ASNT National Fal1 Conference, October, Denver, C0, p. 49 (1978).

3. M. Kuriyama, W. J. Boettinger, and H. E. Burdette, "X-Ray Residual Stress Evaluation by an Energy Dispersive System," Proceedings of a Symposium on Accuracy in Powder Diffraction, NBS Special Pub1. 567, p. 479 (1980).

4. M. Kuriyama, "Procedure for Stress Measurement using Energy Dispersive Diffractometry and High Energy Incident Photons," Symposium on Nondestructive Measurement of Wheel/Axle Residual Stress, DOT Publication (in press).

5. C. J. Bechtoldt, R. C. Placious, W. J. Boettinger and M. Kuriyama, "X-Ray Residual Stress Mapping in Industrial Materials by Energy Dispersive Diffractometry," to be published in "Advances in X-ray Analysis," Vol. 25; 30th Annual Denver X-Ray Conference, Denver, Co (1981). 


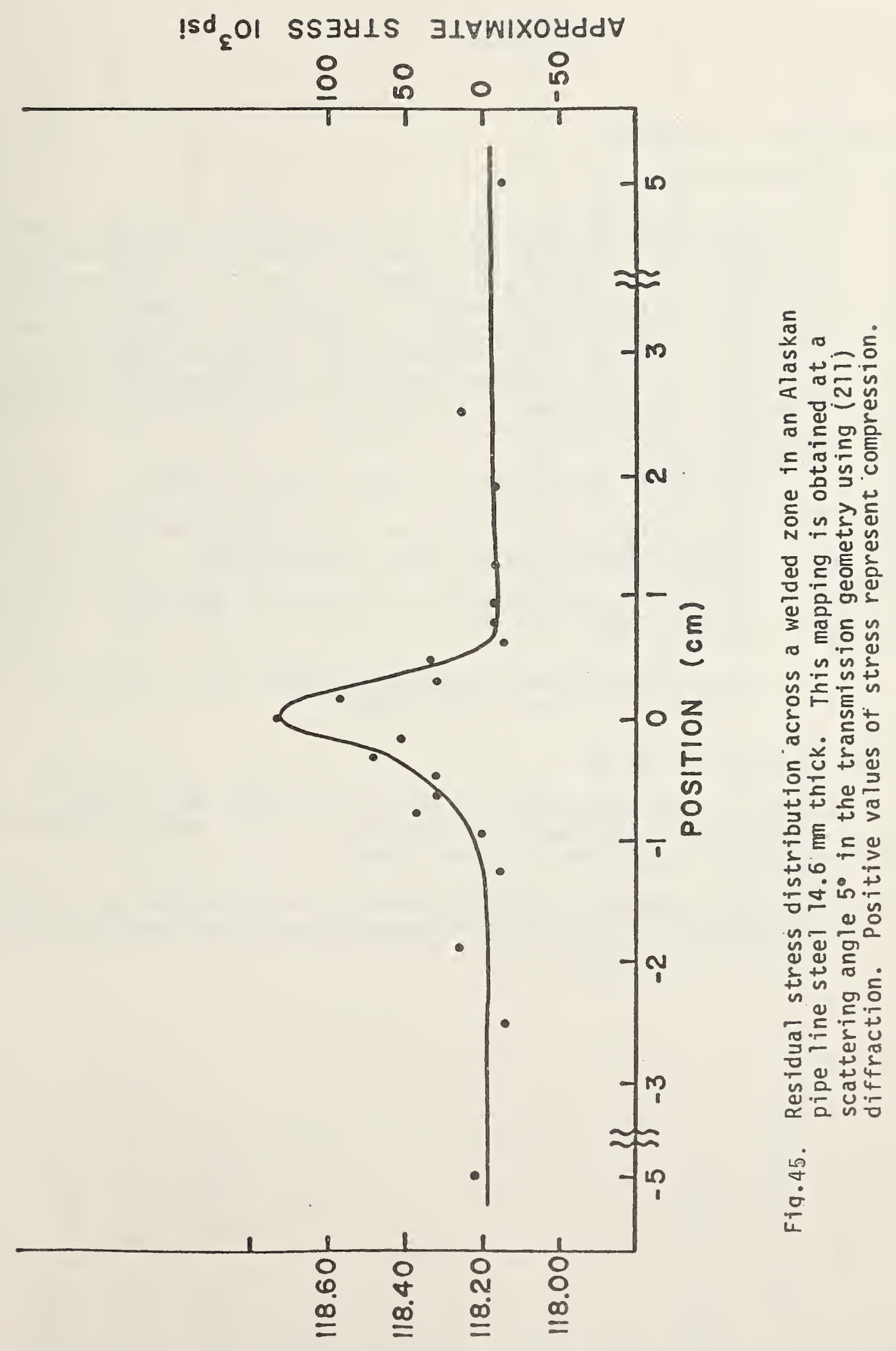

noy Nolllsod Y Y 


\section{APPENDICES}

\section{A. Conferences Sponsored}

1. NDE Meetings at NBS

The NDE program has been active in sponsoring NDE meetings, both symposia and workshops. The purposes behind this significant meeting activity include (1) drawing attention of the industrial community to NDE, (2) providing a good record of the state-of-the-art, (3) providing a forum for information exchange, (4) helping our NBS staff meet individuals active in NDE and learning the status of current work in the field, and (5) providing a mechanism for NDE people to discuss their NDE standards needs. In the past year the Office of NDE played a strong role in the following meetings:

Symposium on-

Ultrasonic Measurements of Stress, April 9, 1981.

Sixth International Symposium on U1trasonic Imaging and Tissue Characterization, May 31-June 3, 1987.

Briefing Workshop on Rapid Solidification Technology, July 1-2, 1981.

Second International Conference on Durability of Building Materials, September 14-16, 1981.

2. NBS Seminars on NDE

A list of NBS-NDE Seminars held during the past year is given in Table VII. Notices of the seminars are distributed broadly in the Washington area. Visitors are welcome and often attend.

This year several of the NDE seminars were transmitted to the Boulder Laboratory via a conference telephone line and the simultaneous use of visuals in Boulder. 


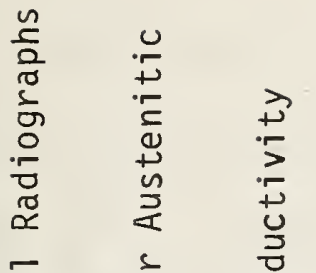

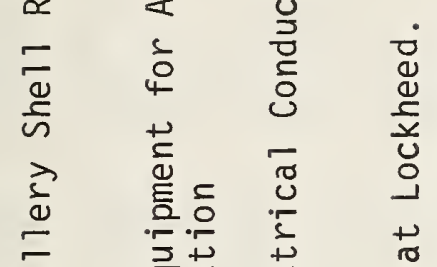

$$
\begin{aligned}
& \begin{array}{cc}
0 \\
\\
0
\end{array}
\end{aligned}
$$

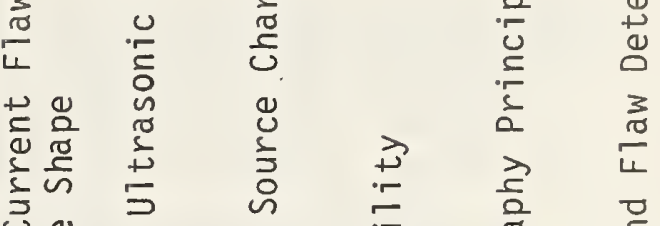

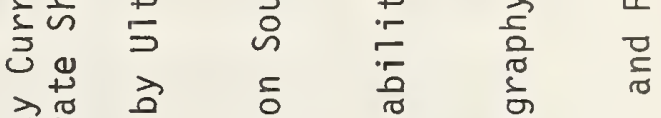

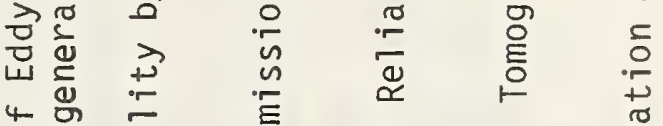

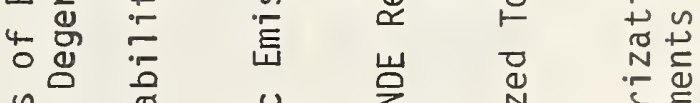

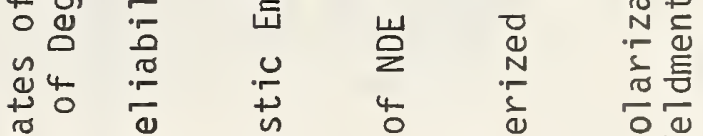

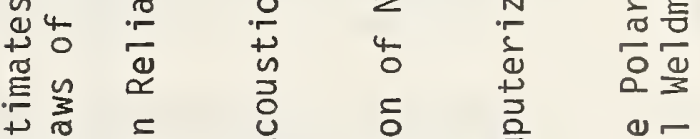

$$
\begin{aligned}
& \text { 苟它 }
\end{aligned}
$$

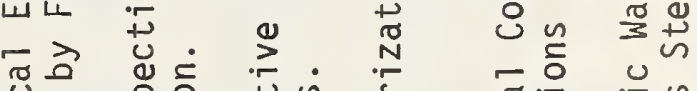

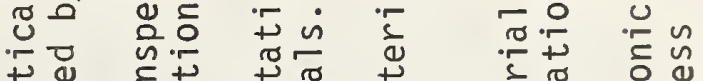

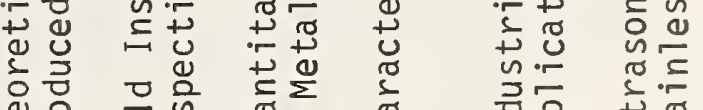

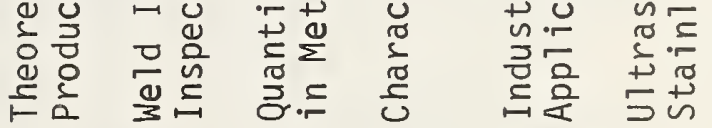

$$
\begin{aligned}
& \vec{~}
\end{aligned}
$$

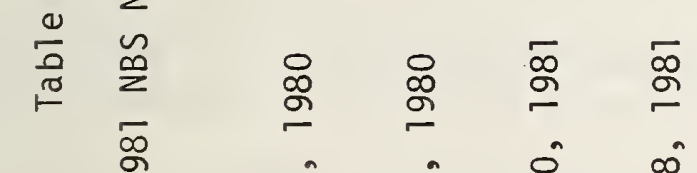

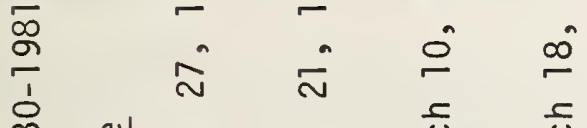

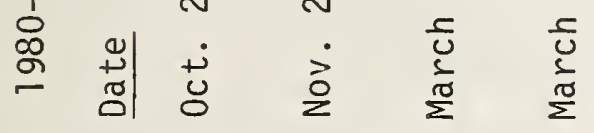

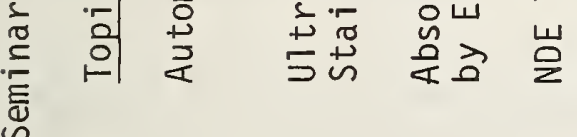

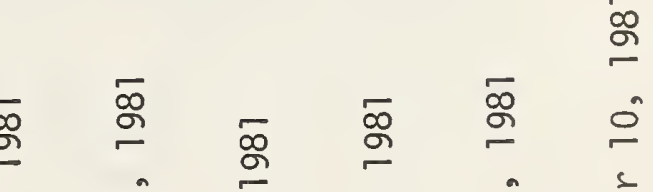

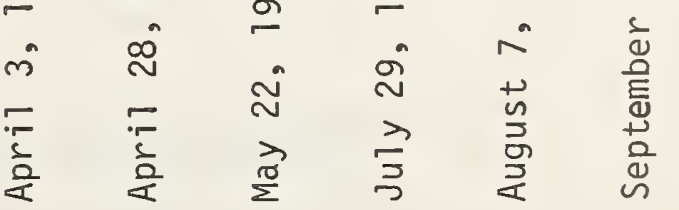

$$
\begin{aligned}
& \stackrel{\dot{0}}{\operatorname{d}}
\end{aligned}
$$

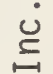

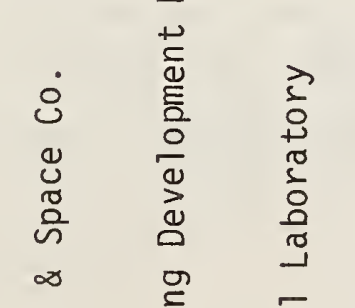

$$
\begin{aligned}
& \text { गे }
\end{aligned}
$$

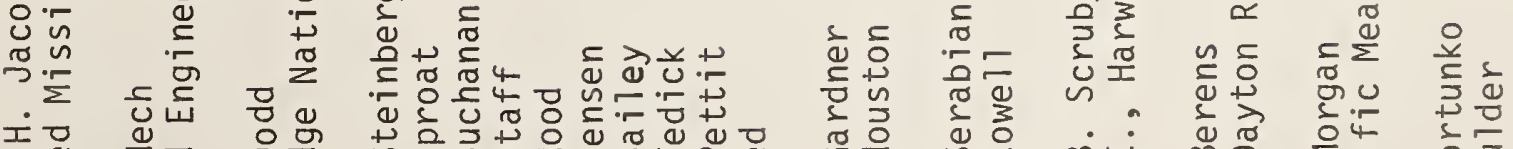

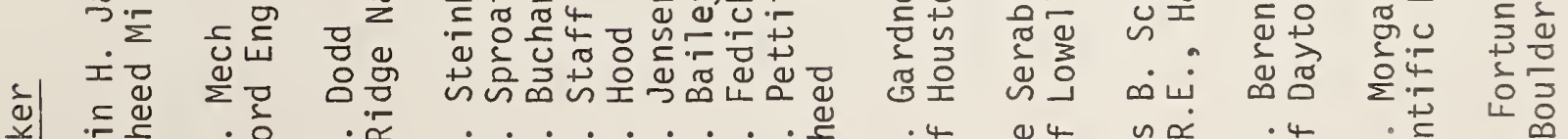

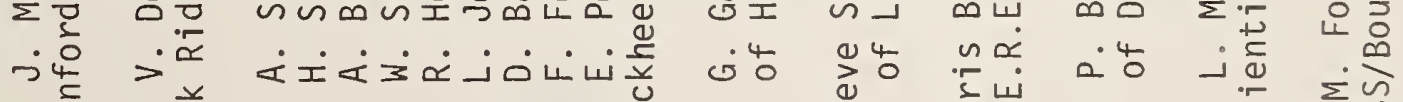

$$
\begin{aligned}
& \text { vir } \text { ن }
\end{aligned}
$$


B. Talks by NDE Office Personnel

1. "Some New Developments in Radiography," H. Berger, 01d Dominion ASNT Section, Richmond, VA, October 28, 1980.

2. "NDE at NBS," Regional Workshop on Research in NDE, H. Berger, University of Houston, Houston, TX, November 10, 1980.

3. "Toward the Nondestructive Characterization of Damage in Composite Materials," L. Mordfin, ASTM Symposium on Damage in Composite Materials: Mechanisms, Accumulation, Tolerance and Characterization, Bal Harbour, FL, November 13, 1980.

4. "Nondestructive Measurement of Resuidual Stress: A Status Report," L. Mordfin, Fifth Workshop on Government Supported R\&D in NDE, Washington, D.C., December 1, 1980.

5. "Materials Testing Equipment and Technology for the 1980's". L. Mordfin, DOC Technical Sales Seminars, Budapest, Hungary; Bucharest, Romania; Prague, Czechoslovakia, January 20-28, 1981.

6. "The National Bureau of Standards Program on Nondestructive Evaluation," H. Berger, Nondestructive Testing Management Association, Las Vegas, NV, February 4, 1981.

7. "Development of Improved Standards for Leakage Rate Measurements," L. Mordfin, ASME Boiler and Pressure Vessel Committee Subgroup on General Requirements, New York City, NY, March 3, 1981.

8. "Measurement of Residual Stresses: Problems and Opportunities," L. Mordfin, WESTEC '81 Conference, Los Angeles, CA, March 25, 1981.

9. "Standards for Residual Stress Measurement," L. Mordfin, ASTM Symposium on Residual Stress Effects in Fatigue, Phoenix, AZ, May 11, 1981.

10. "Introduction to Residual Stress Measurement," L. Mordfin, Symposium on Nondestructive Measurement of Wheel/Axle Residual Stress, Cambridge, MA, June $16,1981$.

11. "Priority Issue No. 1 - Reliability of Nondestructive Evaluation," L. Mordfin, ASME Pressure Vessels and Piping Conference, Denver, C0, June 23, 1981.

12. "New Developments in Radiography", H. Berger, Philadelphia Section ASNT, Philadelphia, PA, September 3, 1981. 
C. Awards and Recognition

Industrial Research, IR-100 Award

For the third consecutive year an NDE-oriented development was recognized with an IR-100 award. Mr. Thomas M. Proctor of the Mechanical Production Metrology Division received the award for an improved acoustic emission transducer.

The improved acoustic emission transducer accurately measures small surface displacements due to acoustic emission events by converting the surface motion to a voltage signal. The improvements produce a transducer that has a very flat response over its working range ( $50 \mathrm{KHz}$ to $1 \mathrm{MHz}$ ) and sensitivity approaching that of a conventional piezoelectrc transducer.

\section{Department of Commerce Medals}

The Silver Medal of the Department of Commerce was awarded to E. Clayton Teague of the Mechanical Production Metrology Division for technical accomplishments and leadership in measurement of surface texture. Dr. Teague leads the NDE program's activity on optical measurement of surface roughness.

Department of Commerce Bronze Medals were awarded to Donald G. Eitzen of the Mechanical Production Metrology Division for his outstanding leadership in the development and execution of the NBS program in ultrasonic standards, and to Lewis K. Ives of the Metallurgy Division for his outstanding contributions to research in the wear of metals and microstructural analysis.

\section{Professional Society Awards}

The Illuminating Engineering Society of North America presented its Special Presidential Award to Gary Yonemura of the Environmental Design Research Division in recognition and appreciation for his valued research and contributions. Dr. Yonemura is the NDE program's principal investigator for efforts to establish meaningful measures of visual acuity for inspection personnel.

George G. Harman of the Semiconductor Devices and Circuits Division received the Internaional Society for Hybrid Microelectronics' highest technical award for his pioneering efforts in the field of wire-bonding process characterization which significantly reduced field failures resulting from wire-bond problems. Mr. Harman is the author of NBS Special Publication 400-59, "Semiconductor Measurement Technology: Nondestructive Tests Used to Insure the Integrity of Semiconductor Devices, with Emphasis on Acoustic Emission Techniques," September 1979. 
NBS Scientist Named International NDE Conference Chairman

It has been announced that Roger Clough will be the general chairman for the Fifth International Conference on Nondestructive Evaluation in the Nuclear Industry, to be held in San Diego in May, 1982. Clough, a metallurgist in the Metallurgy Division, has been active in NDE and materials research at NBS, most recently with the quantitative acoustic emission method being developed jointly by NBS and the Harwell Atomic Energy Research Establishment. 
D. Publications

1. "Eddy-Current Characterization of Materials and Structures," G. Birnbaum, G. Free, Eds., Proceedings of Symposium held at NBS in September 1979, ASTM STP 722 (1981).

2. "Eddy Current Nondestructive Testing," NBS Special Publication 589, G. M. Free, Ed., Workshop on Eddy Current NDT held at NBS in November 1977 (1981).

3. "Nondestructive Testing In The $80^{\prime} \mathrm{s}, " \mathrm{H}$. Berger, Canadian Society for Nondestructive Testing, 2, No. 7, pp. 1418 (Apri1 1981).

4. "Dimensioning Flaws in Pipeline Girth Welds by Radiographic Methods," R. C. Placious, D. A. Garrett, M. B. Kasen, H. Berger, Materials Evaluation, 39, No. 8, pp. 755-759 (July 1981).

5. "A Basis for Traceable NDE Measurements," D. G. Eitzen, H. Berger, G. Birnbaum, NBSIR 802172 (December 1980).

6. "Technical Activities of the Office of Nondestructive Evaluation," H. Berger and L. Mordfin, Eds., NBSIR-80-2162 (Nov. 1980).

7. "Enhanced Reliability and Reproducibility of Measurements of Machinery Vibrations," L. Mordfin, B. Payne, S. Edelman, Proc. Sixth Machinery Dynamics Seminar, Toronto, Canada, Sept. 1980, pp. $4-0-4-22$.

8. "Reliability of Nondestructive Evaluation," L. Mordfin, Critical Issues in Materials and Mechanical Engineering, ASME, pp. 133-147 (1981).

9. "Measurement of Residual Stresses: Problems and Opportunities," L. Mordfin, Residual Stress for Designers and Metallurgists, ASM, pp. 189-210 (1981).

10. "NDE Standards for Nuclear Power Systems: An NBS Perspective," L. Mordfin, H. Berger, Nondestructive Evaluation in the Nuclear Industry - 1980, ASM, pp. 303-318 (1981).

11. "Criteria for Recommending Lighting Leve1s," G. T. Yonemura, NBSIR 81-2231 (March 1981).

12. "Correlation of Source Microstructure and Acoustic Emission Signals Using Indentation Fatigue and Stress Corrosion Cracking in Aluminum Alloys," R. B. Clough, J. C. Chang, J. P. Travis, Scripta Metallurgica (Apri1 1981).

13. "Cryogenic Properties of Filamentary-Reinforced Composites: An Update," M. B. Kasen, Cryogenic Journal, pp. 323-340 (June 1981).

14. "Interim Report on the Significance of Blunt Flaws in Pipeline Girth Welds," M. B. Kasen, R. P. Mikese11, Proc. International Conference on Pipelines and Energy Piping, Calgary, Alberta, Canada, 0ct. 1980, pp. 329-336 (1980). 
15. "Effect of Blunt Flaws Studied in Pipeline Girth Welds,"

M. B. Kasen, R. P. Mikese11, 011 and Gas Journa1, Vol. 79, No. 13, March 30, 1981, pp. 155-158.

16. "Relationship Between Process Variables, Microstructures and NDE of a Precipitation Hardened Aluminum A11oy," L. J. Swartzendruber, W. J. Boettinger, L. K. Ives, S. R. Corie11, R. Mehrabian, Proc. of NDE: Microstructures and Reliability Prediction, Pittsburgh, PA., October 1980 (1981).

17. "Semiconductor Technology Program Progress Briefs," W. M. Bullis, Ed., NBSIR 81-2230 (March 1981).

18. "Anisotrophic Elastic Constants of A Fiber-Reinforced Boron-Aluminum Composite," S. K. Datta, H. M. Ledbetter, Mechanics of Nondestructive Testing, Proceedings of the Conference on Mechanics of Nondestructive Testing, VPI, Blacksburg, VA, Sept. 1980, pp. 215-230.

19. "Piezoelectric Polymer Probe for Ultrasonic Applications," A. S. Dereggi, S. C. Roth, J. M. Kenney, S. Edelman, G. R. Harris, J. of Acoustical Society of America, 69, 3, pp. 853-859 (March 1981).

20. "Ultrasonic Materials Characterization," H. Berger, M. Linzer, Eds., NBS Special Publication 596, Proceedings of the First International Symposium on U1trasonic Materials Characterization, June 1978 (Nov. 1980).

21. "Laboratory Evaluation of Nondestructive Methods to Measure Moisture in Built-up Roofing Systems," L. Knab, R. Mathey, D. Jenkins, NBS Building Science Series 131 (Jan. 1981).

22. Nondestructive Evaluation Methods for Quality Acceptance of Hardened Concrete in Structures," J. R. Clifton, E. D. Anderson, NBSIR 80-2163 (January 1981).

23. "Nondestructive Examination of A Defective Silver Braze Using Resonance-Neutron Radiography," J. W. Behrens, R. A. Schrack and C. D. Bowman, Nuclear Technology, Vol. 51, No. 1, pp. 78-82 (Nov. 1980).

24. "Piezoelectric and Pyroelectric Applications of Plastics," M. G. Broadhurst, S. Edelman, G. T. Davis, Organic Coatings and Plastics Chemistry, ACS 179th National Meeting, Houston, TX, March 1980, pp. 241-245 (1980).

25. "An U1trasonic Investigation of Precipitation Hardening Phenomena in 2219 Aluminum Alloy," M. Rosen, S. Fick and E. Horowitz, Johns Hopkins U. Center for Materials Research, Semi-Annual Report Sept. 1980 - March 1981 (March 1981).

26. "Summary of Fundamental Developments for Quantitative Acoustic Emission Measurements," D. G. Eitzen, F. R. Breckenridge, R. B. Clough, E. R. Fuller, N. N. Hsu, J. A. Simmons, Interim Report - EPRI NP-1.877 (June 1981). 
27. "1980 Annual Report: Technical Contributions to the Development of Incipient Fault Detection/Location Instrumentation," W. E. Anderson, J. D. Ramboz, NBSIR 81-2235 (March 1981).

28. "Auto: A Real Time Diffractometer Control System," R. L. Snyder, C. R. Hubbard, N. C. PanagiotopouTos, NBSIR 81-2229 (February 1981).

29. "Nuclear Safeguard Interrogation of Waste Containers with Resonant Neutrons," Y. Cheng, D. A. Garrett, NDT for Energy Progress, ASNT National Fall Conference, Houston, TX, pp. 97-99 (October 1980).

30. "The Use of X-Ray and Neutron Radiography to Evaluate Lithium/Iodine Pacemaker Batteries," D. A. Garrett, R. C. Stinebring, NDT for Energy Progress, ASNT National Fall Conference, Houston, TX, pp. 215-219 (October 1980).

31. "Holographic NDE: Status and Future," C. M. Vest, NBS-GCR-81-318 (May 1981).

32. "Feasibility of Crack Growth Characterization in a Plate Geometry," H. Wadley, United Kingdom Atomic Energy Authority Harwell for NBS (October 1980).

33. "Ultrasonic Reflectivity Imaging in Three Dimensions: Exact Inverse Scattering Solutions for Plane, Cylindrical, and Spherical Apertures," S. J. Norton, M. Linzer, IEEE Transactions on Biomedical Engineering, Vol. BME-28, No. 2, pp. 202-220 (February 1981).

34. "Optical Interferometric Visualization and Computerized Reconstruction of UItrasonic Fields," F. P. Higgins, S. J. Norton, M. Linzer, J. Acoust. Soc. Am., 68, 4, pp. 1169-1176 (October 1980).

35. "Characterization and Calibration of Acoustic Emission Sensors," N. Hsu, F. Breckenridge, Materials Evaluation, Vol. 39, No. 1, pp. 60-68 (January 1981).

36. "AE Signal Analysis - Laboratory Experiments Examining the Physical Processes of Acoustic Emission," N. Hsu, D. G. Eitzen, Proceedings of the Fifth Acoustic Emissior, Symposium, Tokyo, Japan, pp. 67-78 (1980).

37. "Acoustic Emission Signatures and Source Microstructure Using Indentation Fatigue and Stress Corrosion Cracking in Aluminum Alloys," R. B. Clough, J. C. Chang, J. P. Travis, Scripta Metallurgica, Vol. 15, pp. 417-422 (1981).

38. "B-Scan Echo-Amplitude Measurement in Patients with Diffuse Infiltrative Liver Disease," T. H. Shawker, B. Moran, M. Linzer, S. I. Parks, S. P. James, F. W. Stromeyer, J. A. Barranger, J. Clin. Ultrasound, 9, pp. 293-301, (Aug. 1981).

39. "Tomographic Image Reconstruction from Limited Projections Using Iterative Revisions in Image and Transform Spaces," T. Sato, S. J. Norton, M. Linzer, 0. Ideda, M. Hirama, Applied Optics, Vol. 20, No. 3, (February 1981). 
40. "Nondestructive Evaluation of Non-Uniformities in Aluminum Alloy Plate," G. M. Free, L. K. Ives, R. Mehrabian, L. J. Swartzendruber, ASNT Summaries, pp. 39-43, (1980).

41. "High Accuracy Conductivity Measurements in Nonferrous Metals," G. Free, in Eddy-Current Characterization of Materials and Structures, ASTM STP 722, pp. 121-129 (1981).

42. "Dynamic elastic modulus and internal friction in G-10CR and G-11CR fiberglass-cloth-epoxy composites," H. M. Ledbetter, Cryogenics 20, 637-640 (1980).

43. "Temperature dependence of the Young's modulus and internal friction of G-10CR and G-11CR epoxy resins," H. M. Ledbetter and G. Maerz, Cryogenics 20, 655-658, (1980).

44. "Sound velocities and elastic constants of steels 304,310 , and 316, "H. M. Ledbetter, Metal Sci. 14, 595-596, (1980).

45. "Young's modulus of a copper-stabilized niobium-titanium superconductive wire," H. M. Ledbetter, J. C. Moulder, and M. W. Austin, Wire 31 (Jan./Feb.), 27-29 (1981).

46. "Stainless steel elastic constants at low temperatures," H. M. Ledbetter, J. Appl. Phys. 52, 1587-1589 (1981).

47. "A Boundary Integral Equation Method for Calculating the Eddy-Current Distribution in a Long Cylindrical Bar with a Crack," A. H. Kahn and R. Spal in Eddy Current Characterization of Materials and Structures, ASTM Tech. Pub. 722, G. Birnbaum and G. Free, Eds., (1981), pp. 298.

48. "Viscoelastic Characterization of Structural Adhesives via Forced Oscillation Experiments," D. L. Hunston, W. D. Bascom, E. E. Wells, J. D. Fahey, and J. L. Bitner, in Adhesion and Adsorption of Polymers, Polymer Science and Technology Series, L. H. Lee, Ed., p. A 321 (Plenum Press, New York, 1980).

49. "Linear Viscoelastic Properties of Solid Polymers as Modeled by a Simple Epoxy," D. L. Hunston, W. Carter, and J. L. Rushford, in Developments in Adhesives - 2, A. J. Kinloch, Ed. (Applied Science Publishers, Ltd., London, 1981, pp. 125-172).

50. "Viscoelastic Fracture of Structural Adhesives," J. L. Bitner, J. L. Rushford, W. S. Rose, D. L. Hunston, and C. K. Riew, J: of Adhesion, in press.

51. "Optical Techniques for On-line Measurement of Surface Topography," T. V. Vorburger, and E. C. Teague, Precision Engineering $\underline{3}, 67-83$, (1981).

52. "Light Scattering from Manufactured Surfaces," E. C. Teague, T. V. Vorburger, and D. Maystre, Annals of CIRP, September 1981. 
53. "A Basis for Traceable NDE Measurements Part 1," Eitzen, Berger, and Birnbaum, Materials Evaluation, 39, No. 9, p. 797-798, (Aug., 1981).

54. "Moisture detection in Roofing by Nondestructive Means--A State-of-theArt Survey," David R. Jenkins, Robert G. Mathey, and Lawrence I. Knab, NBS Tech Note 1146, JuTy 1981.

55. "Laboratory Evaluation of Nondestructive Methods to Measure Moisture in Built-Up Roofing Systems," NBS Building Science Series 131.

56. "A-E Signal Analysis--Laboratory Experiments Examining the Physical Process of Acoustic Emission," N. N. Hsu and D. G. Eitzen, Proceedings of the Fifth International Acoustic Emission Symposium, Tokyo, November 1980.

57. "Characterization and Calibration of Acoustic Emission Sensors," N. N. Hsu and F. R. Breckenridge, Materials Evaluation, 39, No. 1, pp. 60-68. (Jan. 1981).

58. "Surface-Wave Displacement: Absolute Measurements Using a Capacitive Transducer," Franklin R. Breckenridge and Martin Greenspan, J. Acoust, Soc. Am. 69, No. 4, pp. 1177-1185, Apri1 1981.

59. "NBS: Nondestructive Evaluation of Nonuniformities in 2219 Aluminum Alloy Plate - Relationship to Processing," L. Swartzendruber, W. Boettinger,

L. Ives, S. Coriel1, D. Ballard, D. Laughtin, R. Clough, F. Biancaniel10, P. Blau, J. Cahn. R. Mehrabian, G. Free, H. Berger, and L. Mordfin, NBSIR 80-2069, December 1980.

60. "Neutron Diffraction and Small-Angle Scattering of Nondestructive Probes of the Microstructure of Materials," D. J. Glinka, H. J. Prask and C. S. Choi, Mechanics of Nondestructive Testing, Proceedings of the Conference on Mechanics of Nondestructive Testing, VPI, Blacksburg, VA, Sept. 1980, pp. 143-164. 



\section{FEDERAL LABORATORY \\ TECHNOLOGY APPLICATION ASSESSMENT}

National Bureau of Standards

National Measurement Laboratory

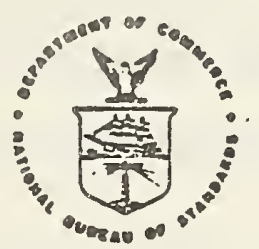

NBS CONICAL TRANSDUCER

A new acoustic emission (AE) transducer, the NBS Conica? Transducer, is under development. It is already changing research and applications approaches in $A E$ because it faithfully measures dynamic displacement, whereas commercially available sensors only detect a disturbance but do not faithfully measure it.

The NBS Conical Transducer follows dynamic normal displacements nearly as well as the standard capacitive transducer used in the NBS calibration service for AE transducers. However, the new device is more rugged than the capacitive transducer and is one to two orders of magnitude more sensitive -. about as sensitive as commercial AE transducers.

STAGE OF DEVELOPMENT

The usefulness of the device has been demonstrated experimentally. Further research of a theoretical nature is planned to optimize the development.

APPLICATIONS

The NBS Conical Transducer is applicable to inservice monitoring of AE on a broad variety of structural components.

\section{PATENT STATUS}

NBS has been granted an IR-100 award for this development. No patent action is contemplated at this time.

PUBLICATIONS

T. Proctor, "Improved Piezoelectric Transducer for Acoustic Emission Signal Reception", Abstract KK-4, J.Acoust. Soc. Am. 68, Suppl. I (Fall 1980). 


\section{FEDERAL LABORATORY \\ TECHNOLOGY APPLICATION ASSESSMENT}

National Bureau of Standards

National Measurement Laboratory

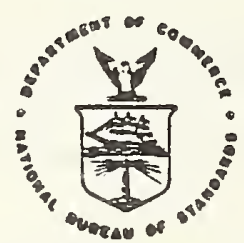

\section{PENETRANT TEST BLOCK}

The NBS Office of Standard Reference Materials has announced the availability of Standard Reference MateriaI (SRM) 1850, a penetrant test block containing uniform "cracks" of known width for checking the sensitivity and perfomance of surface-defect detecting materials and devices, mainly liquid dye penetrants and dye penetrant systems. The test blocks were developed by the Corrosion and Electrodeposition Group of the NBS Center for Materials Science in an effort supported by the NBS Office of Nondestructive Evaluation.

Four cracks approximately $0.2,0.5,1$ and $2 \mathrm{~km}$ wide are formed in a $1 / 2 \mathrm{x} 2 \mathrm{~cm}$ section of an electrodeposited copper-nickel laminate mounted in an epoxy resin disk about $5 \mathrm{~cm}$ in diameter and $1 \mathrm{~cm}$ thick. The depth of each crack is several times its width--beyond the critical depth for showing dye penetrants. Each crack is measured at the center of the blocks with an accuracy of $50 \%$ or $0.1 \mathrm{\mu m}$ whichever is greater. The test block may be used indefinitely as it is easily cleaned and corrosion resistant.

\section{PATENT STATUS}

No patent action will be taken.

\section{PUBLICATIONS}

H. Berger, and L. Mordfin, Eds., "Technical Activities 1980, Office of Nondestructive Evaluation", NBSIR 80-2162, pp. 67-68 (Nov. 1980).

\section{ADDITIONAI INFORMATION}

SRM 1850 may be purchased from the Office of Standard Reference Materials, Room B311, Chemistry Building, National Bureau of Standards, Washington, D.C. 20234, for \$108. Telephone (301) 921-2045, FTS 921-2045. Further details are furnished with the SRM. 
NBS. 114A (REV. 2-8C)

U.S. DEPT. OF COMM.

BIBLIOGRAPHIC DATA

SHEET (See instructions)
1. PUBLICATION OR REPORT NO.

NBSIR $82-2449$
2. Performing Organ. Report No, 3. Publication Date

January 1982

4. TITLE AND SUBTITLE

Technical Activities 1981, Office of Nondestructive Evaluation

5. AUTHOR(S)

H. Berger and L. Mordfin, editors

6. PERFORMING ORGANIZATION (If joint or other than NBS, see instructions)

NATIONAL BUREAU OF STANDARDS

DEPARTMENT OF COMMERCE

WASHINGTON, D.C. 20234

9. SPONSORING ORGANIZATION NAMYE AND COMPLETE ADDRESS (Strect, City, STOTE, ZIP)

7. Contract/Grant No.

8. Type of Report \& Period Covered

10. SUPPLEMENTARY NOTES

[Document describes a computer program; SF-185, FIPS Software Summary, is attached.

11. ABSTRACT (A 200-word or less factual summary of most significant information. If document includes a significant bibliography or literature survey, mention it here)

This is the fourth in a series of annual reports describing the technical activities of the nondestructive evaluation program at the National Bureau of Standards.

12. KEY WORDS (Six to twelve entries; alphabetical order; capitalize only proper names; and separate key words by semicolons) Acoustic emission; eddy currents; imaging; leakage testing; magnetics; material parameters; nondestructive evaluation; optics; penetrants; radiography; and ultrasonics.

13. AVAILABILITY

XXUnlimited

$\square$ For Official Distribution. Do Not Release to NTIS

$\square$ Order From Superintendent of Documents, U.S. Government Printing Office, Washington, D.C. 20402.

[X] Order From National Technical Information Service (NTIS), Springfield, VA. 2216I
14. NO. OF

PRINTED PAGES

\section{4}

15. Price

$\$ 12.00$ 


